\title{
Using Quantitative Spectroscopic Analysis to Determine the Properties and Distances of Type II Plateau Supernovae: SN 2005cs and SN 2006bp
}

\section{Citation}

Dessart, Luc, Stéphane Blondin, Peter J. Brown, Malcolm Hicken, D. John Hillier, Stephen T. Holland, Stefan Immler, et al. 2008. "Using Quantitative Spectroscopic Analysis to Determine the Properties and Distances of Type II Plateau Supernovae: SN 2005cs and SN 2006bp." The Astrophysical Journal 675 (1): 644-69. https://doi.org/10.1086/526451.

\section{Permanent link}

http://nrs.harvard.edu/urn-3:HUL.InstRepos:41399737

\section{Terms of Use}

This article was downloaded from Harvard University's DASH repository, and is made available under the terms and conditions applicable to Open Access Policy Articles, as set forth at http:// nrs.harvard.edu/urn-3:HUL.InstRepos:dash.current.terms-of-use\#OAP

\section{Share Your Story}

The Harvard community has made this article openly available.

Please share how this access benefits you. Submit a story.

\section{Accessibility}


Draft Version OCtOBER 28, 2018

Preprint typeset using $\mathrm{I}_{\mathrm{A}}^{\mathrm{T}} \mathrm{EX}$ style emulateapj v. 10/09/06

\title{
USING QUANTITATIVE SPECTROSCOPIC ANALYSIS TO DETERMINE THE PROPERTIES AND DISTANCES OF TYPE II-PLATEAU SUPERNOVAE: SNe 2005cs AND 2006bp
}

\author{
Luc Dessart $^{1,2}$, Stéphane Blondin ${ }^{3}$, Peter J. Brown ${ }^{4}$, Malcolm Hicken $^{3}$, D. John Hillier ${ }^{5}$, \\ Stephen T. Holland ${ }^{6,7}$, Stefan Immler ${ }^{6,7}$, Robert P. Kirshner ${ }^{3}$, Peter Milne ${ }^{1}$, Maryam Modjaz ${ }^{3}$, \& Peter $^{2}$ \\ W. A. Roming ${ }^{4}$ \\ Draft version October 28, 2018
}

\begin{abstract}
We analyze the Type II Plateau supernovae (SN II-P) 2005cs and 2006bp with the non-LTE model atmosphere code CMFGEN. We fit 13 spectra in the first month for SN 2005cs and 18 for SN 2006bp. Swift ultraviolet photometry and ground-based optical photometry calibrate each spectrum. Our analysis shows both objects were discovered less than 3 days after they exploded, making these the earliest SN II-P spectra ever studied. They reveal broad and very weak lines from highly-ionized fast ejecta with an extremely steep density profile. We identify He II $4686 \AA$ emission in the SN 2006bp ejecta. Days later, the spectra resemble the prototypical Type II-P SN 1999em, which had a supergiant-like photospheric composition. Despite the association of SN 2005cs with possible X-ray emission, the emergent UV and optical light comes from the photosphere, not from circumstellar emission.

We surmise that the very steep density fall-off we infer at early times may be a fossil of the combined actions of the shock wave passage and radiation driving at shock breakout. Based on tailored CMFGEN models, the direct-fitting technique and the Expanding Photosphere Method both yield distances and explosion times that agree within a few percent. We derive a distance to NGC 5194, the host of SN 2005cs, of $8.9 \pm 0.5 \mathrm{Mpc}$ and $17.5 \pm 0.8 \mathrm{Mpc}$ for SN 2006bp in NGC 3953. The luminosity of SN 2006bp is 1.5 times that of SN 1999em, and 6 times that of SN 2005cs. Reliable distances to Type II-P supernovae that do not depend on a small range in luminosity provide an independent route to the Hubble Constant and improved constraints on other cosmological parameters.
\end{abstract}

Subject headings: radiative transfer - stars: atmospheres - stars: supernovae: individual: 2005cs, 2006bp, 1999gi - stars: distances - stars: evolution

\section{INTRODUCTION}

Although radiation represents a negligible fraction of the energy in core-collapse supernova (SN) explosions, most of what we know about these events is inferred from the analysis of spectra and light curves. The gravitational binding-energy of the newly-formed protoneutron star is on the order of $10^{53} \mathrm{erg}$. Within milliseconds after core bounce, a few $\times 10^{52}$ erg of this energy is used to photodissociate the infalling outer iron core. Starting with an electron-neutrino burst when the core reaches nuclear densities, the radiation of neutrinos of all flavors operates over a few tens of seconds after the bounce as the protoneutron star cools, and carries away a few $\times 10^{52} \mathrm{erg}$. These neutrinos are believed to deposit $\sim 10^{51} \mathrm{erg}$ ( $1 \%$ of the total) into the infalling progenitor mantle, reversing the accretion, and to provide the internal and kinetic energy for the SN ejecta (Woosley \& Janka 2005). Only $\sim 10^{49} \mathrm{erg}(0.01 \%$ of the total) is even-

\footnotetext{
${ }^{1}$ Department of Astronomy and Steward Observatory, University of Arizona, Tucson, AZ 85721, USA

2 luc@as.arizona.edu

${ }^{3}$ Harvard-Smithsonian Center for Astrophysics, Cambridge, MA 01238, USA

${ }^{4}$ Pennsylvania State University, Department of Astronomy \& Astrophysics, University Park, PA 16802, USA

${ }^{5}$ Department of Physics and Astronomy, University of Pittsburgh, Pittsburgh, PA 15260

6 Astrophysics Science Division, X-Ray Astrophysics Branch Code 662, NASA Goddard Space Flight Center, Greenbelt, MD 20771, USA

7 Universities Space Research Association, 10211 Wincopin Circle, Columbia MD 21044, USA
}

tually processed into light, that gets radiated with a rate equivalent to a few $\times 10^{8} \mathrm{~L}_{\odot}$ sustained for three months.

Depending on the progenitor radius, the shock emerges at the surface between a few hours and a day after core bounce, with a radiative precursor that is expected to heat and accelerate the surface layers, producing a soft X-ray flash (Chevalier 1982; Ensman \& Burrows 1992; Matzner \& McKee 1999; Blinnikov et al. 2000). Cooling, due to adiabatic expansion and radiative losses, is moderated as time progresses by recombination of hydrogen during the photospheric phase of Type II SN and by non-thermal excitation by unstable isotopes. Although UV emission is significant for the first few days after shock breakout, the spectral energy distribution (SED) subsequently peaks further and further into the red, first in the visual and then in the near-IR, a few months after explosion (Kirshner et al. 1973,1975; Mitchell et al. 2002; Leonard et al. 2002a; Brown et al. 2007).

Core-collapse SN explosions constitute an excellent laboratory for inferring the properties of massive stars at the end of their lives and, indirectly, their pre-SN evolution. As the material expands, the photosphere recedes to deeper and deeper layers of the star's former envelope, allowing the observer to probe all the way from the surface at shock breakout to the innermost layers just outside the compact remnant. The nebular phase, the epoch when the ejecta become optically thin throughout ( 3-4 months after explosion for Type II SNe), permits the inspection of the regions where the blast originated, offering a means to constrain the mechanism and the morphology of the explosion as well as the nucleosyn- 
thetic yields.

Detailed quantitative analyzes focus on the early, photospheric, evolution (Hoëflich 1988; Eastman \& Kirshner 1989; Schmutz et al. 1990; Baron et al 1996, 2000, 2003, 2004, 2007; Mitchell et al. 2002; Dessart \& Hillier 2005a,2006ab), because the ejecta are best suited for "standard" model atmosphere computations: There is an optically-thick base where the radiation thermalizes; there is no contribution from non-thermal excitation by isotopes like ${ }^{56} \mathrm{Ni}$ and its daughter product ${ }^{56} \mathrm{Co}$; electron scattering dominates the opacity; and line and continuum formation is spatially confined. Advances in computer technology permit better handling of non-LocalThermodynamic-Equilibrium (non-LTE) effects and the critical role played by metals is now systematically accounted for (Baron et al. 2004; Dessart \& Hillier 2005a). Non-LTE effects are important even at early times, for example, to reproduce the He I lines observed in optical spectra.

Here, we present a quantitative spectroscopic analysis of the early photospheric-phase evolution of the Type II SNe 2005cs in NGC 5194 and SN 2006bp in NGC 3953, both with plateau-type light curves as shown in Fig. 1. We employ the non-LTE model atmosphere code CMFGEN (Hillier \& Miller 1998; Dessart \& Hillier 2005a), in its steady-state configuration, following a similar approach to Dessart \& Hillier (2006a; hereafter DH06) and their analysis of SN $1999 \mathrm{em}$. A novel component of our study is the use of Swift (Gehrels et al. 2004) UltraViolet/Optical Telescope (UVOT; Roming et al. 2005) ultraviolet photometry that places important constraints on the SED in the UV, the early cooling of the photosphere, and the reddening. We infer from our models that SN 2005cs and SN 2006bp, were discovered only $\sim 2$ days after explosion, the earliest detections after explosion since SN 1987A (Type II peculiar) and SN 1993J (Type IIb; see, e.g., Matheson et al. 2000). As a result of this prompt discovery, we have the opportunity to observe a bright UV spectrum and to see high-excitation lines that have not previously been observed in SN II-P.

Finally, the fine time sampling of our spectra during the first month, along with dense photometric monitoring allows us to make an accurate distance determination. This is a good demonstration of the potential of Type II-P SNe, when coupled with detailed models, to provide accurate extragalactic distances. Unlike the standard approach that depend on the Cepheid period-luminosity relation in the LMC and other nearby galaxies, our method is based on physical models fitted directly to observations, and the distances it provides are independent of all the lower rungs of the distance ladder. We aim to assess the accuracy of distance determinations based on Type II-P SNe, and to refine the value of the Hubble constant $H_{0}$, in the spirit of the earlier efforts of Schmidt et al. (1994). Measurements of the total matter and baryonic content of the Universe via CMB experiments or largescale structure correlations depend on the square of $H_{0}$ (Spergel et al. 2007; Eisenstein et al. 2005). In concordance solutions, these uncertainties affect measurements of the Dark Energy density and its associated equationof-state parameter $w$ (Garnavich et al. 1998; Riess et al 2005; see Macri et al. 2006 for an illustration of the effect an improved accuracy on $H_{0}$ has on $w$ ). A small improvement in our knowledge of $H_{0}$ will lead to a sig- nificant improvement in the understanding the energy content of the Universe.

This paper is structured as follows. In the next section, we present the photometric and spectroscopic datasets. In 3 , we present the model atmosphere code CMFGEN and describe our methods. In 4 we present a quantitative spectroscopic analysis at 13 photospheric-phase epochs for SN 2005cs, and in \$5 analyze 18 epochs for SN 2006bp. In 6 we apply the Expanding Photosphere Method (EPM) as well as a direct-fitting method (in the spirit of the Spectral-fitting Expanding Atmosphere Method, SEAM, of Baron et al. 2000) to infer the distance and the explosion date for both SN events. In 87 we discuss the implications of our results, and in 88 , we conclude.

\section{SUMMARY OF OBSERVATIONS AND DATA REDUCTION}

To sample the early photospheric phase with as many optical observations as possible, we use multiple data sources. For both objects, Swift UVOT photometry is used. Spectra are less frequently observed than the photometry, typically once every few days, and this sets the limit to our analysis. In the next sections, we review the ground-based optical photometry, the optical spectroscopy, the Swift UVOT data.

\subsection{Optical photometry}

UBVr'i' photometry was obtained for SN 2005cs and SN 2006bp at the FLWO $1.2 \mathrm{~m}$ telescope on Mt. Hopkins. The Minicam instrument was used for SN 2005cs while the Keplercam was used for SN 2006bp (for details on cameras and photometric reduction see Hicken, 2007 in preparation). All data were bias subtracted and flat fielded using standard procedures. PSF-fitting photometry was performed using Dophot (Schechter et al. 1993). Host-galaxy subtraction was performed for SN 2006bp. The host-subtracted photometry was approximately 0.02 mag fainter than the photometry from the unsubtracted images, across all bands. A host-galaxy subtraction template was not available for SN 2005cs but the supernova was on a relatively faint and smooth background and observations were taken while SN 2005cs was bright so the absence of host subtraction is negligible. Comparison stars for each SN were calibrated on only one night. However, our confidence in their accuracy is high as other SNe that had been observed on other photometric nights were observed on these nights, and the results are consistent. The comparison stars of these other SNe showed good agreement across all nights (the standard deviation of a typical comparison star across these nights being about $0.015 \mathrm{mag}$ in $\mathrm{V}$ ). We present the photometry obtained for SN 2005cs in Table 1 and in Fig. 1 (left panel), and for SN 2006bp in Table 2 and in Fig. 1 (right panel), where, for completeness, we have also included, where appropriate, the observations obtained by Pastorello et al. (2006; hereafter, P06) and Tsvetkov et al. (2006), and the Swift observations for the three bluest filters.

\subsection{Optical spectroscopy}

Some of the spectra for SN 2005cs presented and analyzed in this paper have been previously published by P06, while a subset for SN 2006bp have been presented by Quimby et al. (2007). We refer the reader to those 

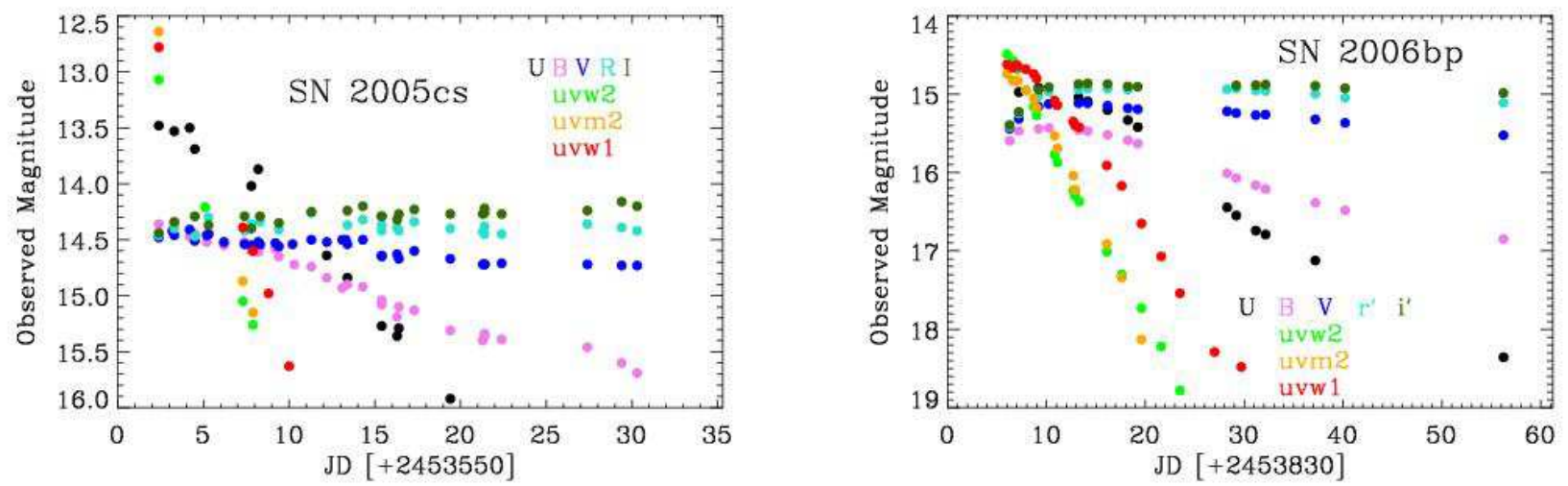

FIG. 1.- Observed light curves for SN 2005cs (left; optical data include the observations of T06, P06, and those of the CfA) and for SN 2006bp (right). Besides optical magnitudes in UBVRI ( $i^{\prime}$ is shown instead of $I$ for SN 2006bp), we also include the Swift UVOT magnitudes in the filters uvw2, uwm2, and uvw1 (corresponding values and photometric errors are given in Table 1 \& 4 for SN 2005cs and Table 2 \& 5 for SN 2006bp, for each filter and date). [See the electronic edition of the Journal for a color version of this figure, and see 2.1 and 2.3 for discussion.]

TABLE 1

JoURNAL OF PHOTOMETRIC OBSERVATIONS OF SN 2005CS

\begin{tabular}{|c|c|c|c|c|c|c|c|c|c|}
\hline UT Date & $\begin{array}{c}\mathrm{JD} \\
(+2,453,000)\end{array}$ & $\overline{\mathrm{U}}$ & B & $\mathrm{V}$ & $\mathrm{R}$ & $\mathrm{I}$ & $\mathrm{r}^{\prime}$ & i' & Source \\
\hline 2005-06-30 & 552.4 & $13.48 \pm 0.05$ & $14.36 \pm 0.05$ & $14.48 \pm 0.02$ & $14.46 \pm 0.04$ & $14.44 \pm 0.04$ & & & P06 \\
\hline 2005-07-01 & 553.2 & & $14.42 \pm 0.02$ & $14.42 \pm 0.02$ & & & $14.68 \pm 0.03$ & $14.34 \pm 0.03$ & CfA \\
\hline 2005-07-01 & 553.3 & $13.53 \pm 0.06$ & $14.36 \pm 0.05$ & $14.46 \pm 0.05$ & $14.40 \pm 0.02$ & $14.34 \pm 0.06$ & & & P06 \\
\hline 2005-07-02 & 554.2 & $13.50 \pm 0.02$ & $14.47 \pm 0.01$ & $14.41 \pm 0.01$ & & & $14.64 \pm 0.02$ & $14.32 \pm 0.02$ & CfA \\
\hline 2005-07-02 & 554.5 & $13.69 \pm 0.10$ & $14.45 \pm 0.04$ & $14.51 \pm 0.03$ & $14.47 \pm 0.03$ & $14.29 \pm 0.10$ & .. & & P06 \\
\hline 2005-07-03 & 555.2 & .. & $14.52 \pm 0.02$ & $14.46 \pm 0.02$ & & & .. & $14.35 \pm 0.02$ & CfA \\
\hline 2005-07-03 & 555.3 & .. & $14.45 \pm 0.02$ & $14.45 \pm 0.02$ & $14.30 \pm 0.02$ & $14.37 \pm 0.05$ & & & T06 \\
\hline 2005-07-04 & 556.2 & .. & $14.55 \pm 0.01$ & $14.52 \pm 0.02$ & & & $14.66 \pm 0.02$ & $14.36 \pm 0.02$ & CfA \\
\hline 2005-07-05 & 557.4 & .. & $14.52 \pm 0.04$ & $14.54 \pm 0.04$ & $14.42 \pm 0.05$ & $14.29 \pm 0.08$ & .. & .. & P06 \\
\hline 2005-07-06 & 557.8 & $14.02 \pm 0.06$ & $14.60 \pm 0.05$ & $14.55 \pm 0.03$ & $14.36 \pm 0.03$ & $14.40 \pm 0.03$ & & & P06 \\
\hline 2005-07-06 & 558.2 & $13.87 \pm 0.02$ & $14.61 \pm 0.02$ & $14.52 \pm 0.02$ & & & $14.60 \pm 0.03$ & $14.33 \pm 0.03$ & CfA \\
\hline 2005-07-06 & 558.3 & .. & $14.57 \pm 0.02$ & $14.54 \pm 0.02$ & $14.34 \pm 0.02$ & $14.29 \pm 0.03$ & & & T06 \\
\hline 2005-07-07 & 559.2 & .. & $14.58 \pm 0.03$ & $14.53 \pm 0.01$ & & & $14.59 \pm 0.02$ & $14.33 \pm 0.01$ & CfA \\
\hline 2005-07-07 & 559.4 & .. & $14.65 \pm 0.04$ & $14.56 \pm 0.09$ & $14.41 \pm 0.09$ & $14.35 \pm 0.11$ & .. & & P06 \\
\hline 2005-07-08 & 560.2 & .. & .. & $14.54 \pm 0.01$ & .. & .. & .. & $14.23 \pm 0.01$ & CfA \\
\hline 2005-07-08 & 560.3 & .. & $14.72 \pm 0.01$ & & & & .. & .. & CfA \\
\hline 2005-07-09 & 561.3 & .. & $14.74 \pm 0.02$ & $14.50 \pm 0.02$ & $14.26 \pm 0.02$ & $14.25 \pm 0.03$ & .. & .. & T06 \\
\hline 2005-07-10 & 562.2 & $14.64 \pm 0.03$ & $14.84 \pm 0.02$ & $14.52 \pm 0.02$ & .. & .. & .. & .. & CfA \\
\hline 2005-07-11 & 563.1 & .. & $14.93 \pm 0.01$ & $14.50 \pm 0.02$ & .. & .. & .. & .. & CfA \\
\hline 2005-07-11 & 563.3 & & & $14.50 \pm 0.02$ & & & .. & .. & T06 \\
\hline 2005-07-11 & 563.4 & $14.84 \pm 0.05$ & $14.90 \pm 0.04$ & $14.54 \pm 0.04$ & $14.37 \pm 0.02$ & $14.24 \pm 0.05$ & .. & .. & $\mathrm{P} 06$ \\
\hline 2005-07-12 & 564.3 & .. & $14.92 \pm 0.02$ & $14.50 \pm 0.02$ & $14.32 \pm 0.02$ & $14.20 \pm 0.03$ & .. & .. & T06 \\
\hline 2005-07-13 & 565.4 & .. & $15.08 \pm 0.05$ & $14.65 \pm 0.04$ & $14.42 \pm 0.04$ & $14.29 \pm 0.06$ & .. & .. & T06 \\
\hline 2005-07-13 & 565.4 & $15.27 \pm 0.06$ & $15.04 \pm 0.05$ & $14.64 \pm 0.02$ & $14.37 \pm 0.03$ & $14.29 \pm 0.05$ & .. & .. & P06 \\
\hline 2005-07-14 & 566.3 & $15.36 \pm 0.06$ & $15.19 \pm 0.02$ & $14.63 \pm 0.02$ & $14.39 \pm 0.02$ & $14.32 \pm 0.03$ & .. & .. & T06 \\
\hline 2005-07-14 & 566.4 & $15.29 \pm 0.05$ & $15.10 \pm 0.05$ & $14.67 \pm 0.02$ & $14.42 \pm 0.04$ & $14.27 \pm 0.02$ & .. & .. & P06 \\
\hline 2005-07-15 & 567.3 & & $15.13 \pm 0.03$ & $14.60 \pm 0.02$ & $14.34 \pm 0.02$ & $14.23 \pm 0.03$ & .. & .. & T06 \\
\hline 2005-07-17 & 569.4 & $15.92 \pm 0.07$ & $15.31 \pm 0.05$ & $14.67 \pm 0.03$ & $14.40 \pm 0.03$ & $14.27 \pm 0.04$ & .. & .. & P06 \\
\hline 2005-07-19 & 571.3 & .. & $15.40 \pm 0.02$ & $14.72 \pm 0.02$ & $14.43 \pm 0.02$ & $14.27 \pm 0.03$ & .. & .. & T06 \\
\hline 2005-07-19 & 571.4 & .. & $15.34 \pm 0.02$ & $14.72 \pm 0.02$ & $14.38 \pm 0.04$ & $14.22 \pm 0.04$ & .. & .. & T06 \\
\hline 2005-07-19 & 571.4 & .. & $15.36 \pm 0.12$ & $14.72 \pm 0.05$ & $14.45 \pm 0.09$ & $14.26 \pm 0.07$ & .. & .. & P06 \\
\hline 2005-07-20 & 572.4 & .. & $15.39 \pm 0.07$ & $14.71 \pm 0.03$ & $14.45 \pm 0.03$ & $14.27 \pm 0.03$ & .. & .. & P06 \\
\hline 2005-07-25 & 577.4 & .. & $15.46 \pm 0.09$ & $14.72 \pm 0.04$ & $14.36 \pm 0.06$ & $14.24 \pm 0.06$ & .. & .. & P06 \\
\hline 2005-07-27 & 579.4 & .. & $15.60 \pm 0.07$ & $14.73 \pm 0.04$ & $14.39 \pm 0.04$ & $14.16 \pm 0.04$ & .. & .. & P06 \\
\hline 2005-07-28 & 580.3 & .. & $15.69 \pm 0.05$ & $14.73 \pm 0.03$ & $14.42 \pm 0.03$ & $14.20 \pm 0.04$ & .. & .. & T06 \\
\hline
\end{tabular}

Note. - Sources for photometric observations are CfA, P06 and Tsvetkov et al. (2006; T06). For the distance determinations performed in 46.1 we use the photometry corresponding to the dates on which spectroscopic observations were obtained. If there is no photometry on the date of a spectrum, we linearly interpolate the slowly varying magnitude obtained at bracketing dates. 
TABLE 2

JOURNAL OF PHOTOMETRIC OBSERVATIONS OF SN 2006BP

\begin{tabular}{|c|c|c|c|c|c|c|}
\hline UT Date & $\begin{array}{c}\text { JD } \\
(+2,453,000)\end{array}$ & $\mathrm{U}$ & B & $\mathrm{V}$ & r' & $\mathrm{i}^{\prime}$ \\
\hline 2006-04-10 & 836.27 & & $15.59 \pm 0.02$ & $15.44 \pm 0.02$ & $15.42 \pm 0.02$ & $15.39 \pm 0.02$ \\
\hline 2006-04-11 & 837.20 & $14.97 \pm 0.03$ & $15.47 \pm 0.02$ & $15.31 \pm 0.02$ & $15.24 \pm 0.02$ & $15.23 \pm 0.02$ \\
\hline 2006-04-13 & 839.20 & $14.92 \pm 0.03$ & $15.44 \pm 0.02$ & $15.16 \pm 0.02$ & $15.03 \pm 0.02$ & $14.94 \pm 0.02$ \\
\hline $2006-04-14$ & 840.23 & & $15.43 \pm 0.02$ & $15.12 \pm 0.02$ & $14.97 \pm 0.02$ & $14.91 \pm 0.01$ \\
\hline 2006-04-17 & 843.24 & $15.04 \pm 0.03$ & $15.46 \pm 0.02$ & $15.11 \pm 0.02$ & $14.94 \pm 0.02$ & $14.87 \pm 0.02$ \\
\hline 2006-04-18 & 844.16 & $15.09 \pm 0.03$ & $15.47 \pm 0.02$ & $15.12 \pm 0.02$ & $14.93 \pm 0.02$ & $14.86 \pm 0.02$ \\
\hline 2006-04-20 & 846.17 & $15.20 \pm 0.02$ & $15.52 \pm 0.02$ & $15.15 \pm 0.02$ & $14.93 \pm 0.02$ & $14.87 \pm 0.02$ \\
\hline 2006-04-22 & 848.22 & $15.33 \pm 0.03$ & $15.59 \pm 0.02$ & $15.18 \pm 0.02$ & $14.94 \pm 0.02$ & $14.90 \pm 0.02$ \\
\hline 2006-04-23 & 849.23 & $15.42 \pm 0.03$ & $15.63 \pm 0.02$ & $15.19 \pm 0.02$ & & $14.91 \pm 0.02$ \\
\hline 2006-05-02 & 858.27 & $16.44 \pm 0.03$ & $16.01 \pm 0.02$ & $15.22 \pm 0.02$ & $14.93 \pm 0.02$ & \\
\hline 2006-05-03 & 859.19 & $16.55 \pm 0.04$ & $16.07 \pm 0.02$ & $15.24 \pm 0.02$ & $14.93 \pm 0.02$ & $14.89 \pm 0.02$ \\
\hline 2006-05-05 & 861.17 & $16.74 \pm 0.04$ & $16.16 \pm 0.02$ & $15.27 \pm 0.02$ & $14.95 \pm 0.02$ & $14.88 \pm 0.02$ \\
\hline 2006-05-06 & 862.16 & $16.79 \pm 0.05$ & $16.21 \pm 0.02$ & $15.26 \pm 0.02$ & $14.96 \pm 0.02$ & $14.88 \pm 0.02$ \\
\hline 2006-05-11 & 867.19 & $17.12 \pm 0.06$ & $16.39 \pm 0.03$ & $15.32 \pm 0.02$ & $15.00 \pm 0.02$ & $14.89 \pm 0.02$ \\
\hline 2006-05-14 & 870.20 & & $16.48 \pm 0.04$ & $15.37 \pm 0.02$ & $15.05 \pm 0.02$ & $14.92 \pm 0.02$ \\
\hline 2006-05-30 & 886.23 & $18.36 \pm 0.07$ & $16.85 \pm 0.02$ & $15.52 \pm 0.02$ & $15.11 \pm 0.02$ & $14.98 \pm 0.02$ \\
\hline
\end{tabular}

Note. - Log of CfA photometric observations. For the distance determination done in 6.2 we only use the $B$, $V$, and $i^{\prime}$ magnitudes. We use photometric data on the dates of the spectra, or, if there is no photometry on the day of a spectrum, linearly interpolate between magnitudes obtained at bracketing dates.

TABLE 3

Journal OF SPECTROSCOPIC OBSERVATIONS OF SNE 2005CS AND 2006BP WiTh FLWO 1.5 M+FAST

\begin{tabular}{|c|c|c|c|c|c|c|c|c|}
\hline $\mathrm{UT}_{\text {Date }}^{\mathrm{a}}$ & $\begin{array}{c}\mathrm{JD}^{\mathrm{a}} \\
(+2,453,000)\end{array}$ & $\begin{array}{l}\text { Epoch } \\
\text { (d) }\end{array}$ & $\begin{array}{c}\Delta \mathrm{PA}^{\mathrm{c}} \\
\left({ }^{\circ}\right)\end{array}$ & Airmass & Flux stds. ${ }^{d}$ & $\begin{array}{l}\text { Seeinge } \\
\left({ }^{\prime \prime}\right)\end{array}$ & $\begin{array}{l}\text { Exp. time } \\
\text { (s) }\end{array}$ & Observer $^{f}$ \\
\hline \multicolumn{9}{|c|}{ SN 2005cs } \\
\hline $2005-06-30.23$ & 551.73 & 4 & 1.1 & 1.31 & BD28, BD17 & $\cdots$ & 1200 & $\mathrm{RH}$ \\
\hline $2005-07-01.27$ & 552.77 & 5 & 5.0 & 1.53 & $\mathrm{BD} 28, \mathrm{BD} 17$ & 4 & 900 & $\mathrm{RH}$ \\
\hline $2005-07-02.23$ & 553.73 & 6 & 3.1 & 1.34 & BD28, BD26 & 2 & 1200 & $\mathrm{RH}$ \\
\hline $2005-07-03.15$ & 554.65 & 7 & 32.7 & 1.09 & F34, BD33 & 2 & 720 & RH \\
\hline $2005-07-04.16^{\mathrm{g}}$ & 555.66 & 8 & 85.4 & 1.11 & BD28,BD17 & 1 & 1200 & $\mathrm{JG}$ \\
\hline $2005-07-04.18^{\mathrm{g}}$ & 555.68 & 8 & 78.4 & 1.15 & BD28,BD17 & 1 & 1200 & $\mathrm{JG}$ \\
\hline 2005-07-05.18 & 556.68 & 9 & 8.9 & 1.16 & $\mathrm{~F} 34, \mathrm{BD} 17$ & 1 & 1200 & $\mathrm{JG}$ \\
\hline 2005-07-06.17 & 557.67 & 10 & 5.2 & 1.15 & $\mathrm{BD} 28, \mathrm{BD} 17$ & 1 & 1200 & $\mathrm{JG}$ \\
\hline $2005-07-07.15$ & 558.65 & 12 & 1.8 & 1.11 & BD28, BD17 & 1 & 1200 & $\mathrm{EF}$ \\
\hline 2005-07-09.19 & 560.69 & 13 & 0.7 & 1.24 & $\mathrm{BD} 28, \mathrm{BD} 17$ & $1-2$ & 1200 & $\mathrm{~PB}$ \\
\hline $2005-07-10.18$ & 561.68 & 14 & 1.1 & 1.22 & $\mathrm{BD} 28, \mathrm{BD} 17$ & $1-2$ & 1200 & $\mathrm{~PB}$ \\
\hline 2005-07-11.18 & 562.68 & 15 & 2.9 & 1.23 & $\mathrm{BD} 28, \mathrm{BD} 17$ & $1-2$ & 1200 & $\mathrm{MC}$ \\
\hline $2005-07-12.16$ & 563.66 & 16 & 2.8 & 1.17 & $\mathrm{BD} 28, \mathrm{BD} 17$ & $1-2$ & 1200 & $\mathrm{MC}$ \\
\hline 2005-07-28.17 & 579.67 & 32 & 1.0 & 1.38 & $\mathrm{BD} 28, \mathrm{BD} 17$ & $1-2$ & 1200 & PB \\
\hline $2005-07-29.15$ & 580.65 & 33 & 2.1 & 1.30 & $\mathrm{BD} 28, \mathrm{BD} 17$ & $2-3$ & 960 & $\mathrm{MC}$ \\
\hline $2005-12-25.56$ & 730.04 & 182 & 5.0 & 1.11 & BD28, BD17 & 1 & 1800 & $\mathrm{MC}$ \\
\hline \multicolumn{9}{|c|}{ SN 2006bp } \\
\hline $2006-04-20.30$ & 845.80 & 12 & 12.3 & 1.13 & F34, HD84 & 1.5 & 1200 & $\mathrm{WP}$ \\
\hline $2006-04-21.21$ & 846.71 & 13 & 3.5 & 1.07 & F34, HD84 & 1.5 & 1200 & WP \\
\hline 2006-04-24.19 & 849.69 & 16 & 67.9 & 1.08 & $\mathrm{~F} 34, \mathrm{BD} 26$ & 1.5 & 1500 & $\mathrm{JH}$ \\
\hline $2006-05-01.35$ & 856.85 & 23 & 0.8 & 1.41 & F34, HD84 & 1.5 & 780 & WB \\
\hline 2006-05-02.14 & 857.64 & 24 & 3.8 & 1.10 & F34, HD84 & $1.5-2$ & 1200 & WB \\
\hline 2006-05-03.22 & 858.72 & 25 & 64.4 & 1.08 & F34, HD84 & $1.5-2$ & 1200 & TG \\
\hline $2006-05-04.15$ & 859.65 & 26 & 2.8 & 1.09 & $\mathrm{~F} 34, \mathrm{HD} 84$ & $1.5-2$ & 1200 & TG \\
\hline 2006-05-07.19 & 862.69 & 29 & 12.2 & 1.07 & $\mathrm{~F} 34, \mathrm{BD} 17$ & $1.5-2$ & 1200 & WP \\
\hline
\end{tabular}

Note. - All the above spectra were obtained with the $1.5 \mathrm{~m}$ Tillinghast telescope using the FAST spectrograph (Fabricant et al. 1998). The grating used yields a resolution of $\sim 7 \AA$. The observed wavelength range roughly spans $3500-7400 \AA$. (See $\$ 2.2$ for discussion).

${ }^{\mathrm{a}}$ UT Date and Julian Date at midpoint of observations. ${ }^{\mathrm{b}}$ Epoch of spectrum relative to the estimated explosion date. ${ }^{\mathrm{c}}$ Absolute difference between the observed position angle and average parallactic angle over the course of the observation. ${ }^{\mathrm{d}}$ Standard star pairs used for flux calibration: $\mathrm{BD} 17=\mathrm{BD}+17^{\circ} 4708 ; \mathrm{BD} 26=\mathrm{BD}+26^{\circ} 2606 ; \mathrm{BD} 28=\mathrm{BD}+28^{\circ} 4211 ; \mathrm{BD} 33=\mathrm{BD}+33^{\circ} 2642 ; \mathrm{F} 34=\mathrm{Feige}$ 34; HD84 = HD 84937. ${ }^{\mathrm{e}}$ Seeing is based upon estimates by the observers. ${ }^{\mathrm{f}}$ Observers: $\mathrm{EF}=\mathrm{E} . \mathrm{Falco} ; \mathrm{JG}=\mathrm{J}$. Gallagher; JH $=$ J. Hernandez; $\mathrm{MC}=\mathrm{M}$. Calkins; $\mathrm{PB}=\mathrm{P}$. Berlind; $\mathrm{RH}=\mathrm{R}$. Hutchins; TG $=\mathrm{T}$. Groner; WB $=\mathrm{W}$. Brown; WP $=$ W. Peters. ${ }^{\mathrm{g}} \mathrm{Both}$ spectra of SN 2005cs on UT 2005-07-04 were combined into a single spectrum for plotting and modeling purposes. 
papers for more information on those spectra. In the analysis section of SN 2005cs, we indicate this source as P06, wherever appropriate, while in the analysis section of SN 2006bp, we indicate this source as Quimby.

All the other spectra were obtained with the $1.5 \mathrm{~m}$ Tillinghast telescope at the Fred Lawrence Whipple Observatory (FLWO) using the low-dispersion FAST spectrograph (Fabricant et al. 1998). Observational details of the spectra are listed in Table 3. The FAST data were all reduced in the same manner, using standard $\mathrm{IRAF}^{8}$ routines for CCD processing and spectrum extraction. All the spectra were extracted using the optimal algorithm of Horne (1986). The spectra are then wavelength calibrated using calibration-lamp spectra; the calibration is later checked against night sky lines and adjusted accordingly. We used our own IDL procedures for flux calibration. These include the removal of telluric lines using the well-exposed continua of the spectrophotometric standard stars (Wade \& Horne 1988; Matheson et al. 2000).

The relative spectrophotometry is good to about $5 \%$, when done at the parallactic angle (Matheson et al. 2007, in prep.), but no attempt was made to determine an absolute flux calibration. To avoid second-order light contamination, we calibrated each spectrum using two standard stars of different colors (see Table 3). In general, the spectra were observed at the parallactic angle to minimize the effects of atmospheric differential refraction (Filippenko 1982). When this is not the case, the spectra can suffer from a relative flux depletion at bluer wavelengths, especially when observed at high airmass ( $\sec z>1.1$, where $z$ is the zenith angle). In Table 3 we list the angle in degrees, $\triangle \mathrm{PA}$, between the observed position angle and the parallactic angle for each spectrum, along with the airmass. When $\Delta \mathrm{PA}>10^{\circ}$, the relative spectrophotometry is less accurate.

Around maximum light, both SNe 2005cs and 2006bp were bright with respect to their host galaxy. Nevertheless, the earliest (June $30^{\text {th }}$ UT) and latest (Dec $25^{\text {th }}$ UT) FAST spectra of SN 2005cs have substantial galaxy background, and the relative flux calibration is affected by this contamination.

Finally, all SN 2005cs spectra presented here have been de-redshifted, adopting our own measurement of $466 \pm 35 \mathrm{~km} \mathrm{~s}^{-1}$ of the recession velocity of NGC 5194, while for SN 2006bp and NGC 3953, we adopt $1280 \mathrm{~km} \mathrm{~s}^{-1}$ (Quimby et al. 2007; Verheijen \& Sancisi 2001; a comparable value of $1053 \mathrm{~km} \mathrm{~s}^{-1}$ is given by Ho et al. 1997).

\subsection{Swift UVOT photometry}

Swift UVOT photometry allows us to track the ultraviolet color evolution of $\mathrm{SNe}$ from a few days to a few weeks after shock breakout. Observations of both SN $2005 \mathrm{cs}$ and SN 2006bp were obtained with Swift UVOT (Gehrels et al. 2004; Roming et al. 2005; Poole et al. 2007) at multiple epochs covering from just hours after discovery until the UV light had faded to the background emission level of the SN environment. The Swift UVOT

\footnotetext{
8 IRAF is distributed by the National Optical Astronomy Observatories, operated by the Association of Universities for Research in Astronomy, Inc., under contract to the National Science Foundation of the United States.
}

data for SNe 2005cs and 2006bp, originally presented in Brown et al. (2007) and Immler et al. (2007), have been reanalyzed using the improved calibration of Poole et al. (2007). The magnitudes in Table 4 and in Table 5 are in the UVOT system, distinguished by lower case letters. The UVOT $u, b$, and $v$ are close to the Johnson $U B V$; however, the UVOT $u$ band is bluer than the Johnson $U$ so there can be significant differences at early times when the SNe are blue. The $b$ and $v$ agree well with their Johnson counterparts. The magnitudes and flux densities were measured using a $5^{\prime \prime}$ aperture source region, corresponding to that used to calibrate the zeropoints and coincidence loss corrections, using uvotmaghist. Here, we subtract the background light by means of late-time template images, selecting a background region with the same average count rate per pixel as in the $5^{\prime \prime}$ source region. When the background subtracted count rate drops below that of the background (as measured in the template images) we report the magnitude of the background as an upper limit. The errors reported include the systematic errors in the zeropoints.

We use the flux equivalent of the Swift UVOT magnitudes given in Table 4 \& 5] accounting for the different (and varying) energy distribution of the supernova by adding an additional systematic error of $10 \%$ in quadrature with the statistical error. We plot these fluxes at the effective wavelengths of the UVOT filters along with appropriately reddened synthetic flux distributions computed with the non-LTE model atmosphere code CMFGEN (see $\S 3$, $\S 4$, and $\S 5$. Swift UVOT filters $u v w 2, u v m 2, u v w 1, u, b$, and $v$ have their effective wavelength at $2030 \AA, 2231 \AA, 2634 \AA, 3501 \AA, 4329 \AA$, and $5402 \AA$ (Poole et al. 2007).

\section{THEORETICAL METHODOLOGY}

The analysis of SNe 2005cs and SN 2006bp is analogous to that presented in DH06, which analyzed SN $1999 \mathrm{em}$ at 8 epochs in the first 45 days after explosion. That paper determined its distance by means of various EPM-based approaches (Kirshner \& Kwan 1974; Eastman \& Kirshner 1989; Schmidt et al. 1994; Eastman et al. 1996; Hamuy et al. 2001; Leonard et al. 2002a; Elmhamdi et al. 2003; Dessart \& Hillier 2005b) and another method akin to the SEAM (Baron et al. 2000). Here, we have two advantages: Swift observations monitor the UV light and we have more densely sampled data. Swift provides additional constraints on the reddening, the temperature, and the ionization state of the ejecta at early epochs. Our analysis covers 13 epochs in ( 30 days) for SN 2005cs and 18 epochs in ( $\sim 45$ days) for SN 2006bp, providing better tracking of the spectral and photometric evolution of each.

Time-dependent terms in the statistical- and radiativeequilibrium equations become important a few weeks after explosion (Utrobin \& Chugai 2005; Dessart \& Hillier $2007 \mathrm{a}, \mathrm{b})$. We avoid that complication by focusing on the early-time photospheric phase. We know that assuming a steady state in the statistical and radiative equilibrium equations produces a prediction of the ejecta ionization state that is somewhat below its true level, that He I lines persist over a longer period than we predict, and that strong Balmer lines at late times are a clear sign of the epoch when this assumption is no longer valid.

CMFGEN solves the radiative transfer equation in the 
TABLE 4

Swift UVOT РнотомеTRY OF SN 2005Cs

\begin{tabular}{|c|c|c|c|c|c|c|c|}
\hline UT Date & JD & $u v w 2$ & uvm 2 & $u v w 1$ & $u$ & $b$ & $v$ \\
\hline $2005-06-30$ & 2453552.4 & $13.07 \pm 0.03$ & $12.64 \pm 0.03$ & $12.78 \pm 0.03$ & $\ldots$ & $\ldots$ & $14.63 \pm 0.03$ \\
\hline $2005-07-03$ & 2453555.1 & $14.21 \pm 0.03$ & $\ldots$ & $\ldots$ & $\ldots$ & $\ldots$ & $14.63 \pm 0.06$ \\
\hline 2005-07-05 & 2453557.3 & $15.05 \pm 0.04$ & $14.87 \pm 0.04$ & $14.39 \pm 0.04$ & $13.64 \pm 0.03$ & $14.75 \pm 0.03$ & $14.68 \pm 0.03$ \\
\hline $2005-07-06$ & 2453557.9 & $15.26 \pm 0.04$ & $15.15 \pm 0.04$ & $14.60 \pm 0.04$ & $13.75 \pm 0.03$ & $14.76 \pm 0.03$ & $14.66 \pm 0.03$ \\
\hline $2005-07-07$ & 2453558.8 & $>15.7$ & $>15.7$ & $14.98 \pm 0.04$ & $13.85 \pm 0.03$ & $14.76 \pm 0.02$ & $14.64 \pm 0.03$ \\
\hline $2005-07-08$ & 2453560.0 & $>15.7$ & $>15.7$ & $15.63 \pm 0.05$ & $14.14 \pm 0.03$ & $14.78 \pm 0.03$ & $14.59 \pm 0.03$ \\
\hline 2005-07-09 & 2453560.6 & $>15.7$ & $>15.7$ & $>15.7$ & $14.32 \pm 0.04$ & $14.78 \pm 0.04$ & $14.58 \pm 0.04$ \\
\hline $2005-07-10$ & 2453562.0 & $>15.7$ & $>15.7$ & $>15.7$ & $14.67 \pm 0.04$ & $14.87 \pm 0.05$ & $14.63 \pm 0.05$ \\
\hline 2005-07-11 & 2453562.8 & $>15.7$ & $>15.7$ & $>15.7$ & $14.92 \pm 0.05$ & $14.98 \pm 0.05$ & $14.70 \pm 0.05$ \\
\hline 2005-07-13 & 2453564.5 & $>15.7$ & $>15.7$ & $>15.7$ & $\ldots$ & $\ldots$ & $14.67 \pm 0.05$ \\
\hline 2005-07-14 & 2453565.5 & $>15.7$ & $>15.7$ & $>15.7$ & $15.43 \pm 0.05$ & $15.21 \pm 0.04$ & $14.74 \pm 0.04$ \\
\hline $2005-07-17$ & 2453568.5 & $>15.7$ & $>15.7$ & $>15.7$ & $>15.8$ & $15.48 \pm 0.05$ & $14.76 \pm 0.05$ \\
\hline $2005-07-22$ & 2453573.5 & $>15.7$ & $>15.7$ & $>15.7$ & $>15.8$ & $15.62 \pm 0.06$ & $14.90 \pm 0.05$ \\
\hline 2005-09-14 & 2453628.5 & $>15.7$ & $>15.7$ & $>15.7$ & $>15.8$ & $16.33 \pm 0.06$ & $14.95 \pm 0.04$ \\
\hline
\end{tabular}

TABLE 5

Swift UVOT РнOTOMETRY OF SN 2006BP

\begin{tabular}{|c|c|c|c|c|c|c|c|}
\hline UT Date & $\mathrm{JD}$ & $u v w 2$ & uvm2 & $u v w 1$ & $u$ & $b$ & $v$ \\
\hline 2006-04-10 & 2453836.0 & $14.49 \pm 0.03$ & $14.74 \pm 0.03$ & $14.62 \pm 0.03$ & $14.61 \pm 0.02$ & $15.75 \pm 0.02$ & $15.63 \pm 0.03$ \\
\hline 2006-04-11 & 2453836.6 & $14.57 \pm 0.04$ & $14.83 \pm 0.05$ & $14.66 \pm 0.04$ & $14.60 \pm 0.04$ & $15.60 \pm 0.04$ & $15.52 \pm 0.05$ \\
\hline 2006-04-11 & 2453836.9 & $14.62 \pm 0.03$ & $14.83 \pm 0.04$ & $14.63 \pm 0.03$ & $14.55 \pm 0.03$ & $15.59 \pm 0.03$ & $15.52 \pm 0.03$ \\
\hline 2006-04-11 & 2453837.1 & $14.68 \pm 0.03$ & $14.83 \pm 0.04$ & $14.64 \pm 0.04$ & $14.58 \pm 0.03$ & $15.53 \pm 0.03$ & $15.44 \pm 0.04$ \\
\hline 2006-04-12 & 2453837.9 & $14.95 \pm 0.03$ & $14.95 \pm 0.04$ & $14.68 \pm 0.03$ & $14.49 \pm 0.03$ & $15.52 \pm 0.02$ & $15.31 \pm 0.03$ \\
\hline 2006-04-13 & 2453838.7 & $15.16 \pm 0.03$ & $15.05 \pm 0.03$ & $14.74 \pm 0.03$ & $14.51 \pm 0.02$ & $15.47 \pm 0.02$ & $15.25 \pm 0.03$ \\
\hline 2006-04-13 & 2453839.0 & $15.27 \pm 0.03$ & $15.17 \pm 0.04$ & $14.80 \pm 0.03$ & $14.52 \pm 0.03$ & $15.43 \pm 0.02$ & $15.18 \pm 0.03$ \\
\hline 2006-04-15 & 2453840.8 & $15.77 \pm 0.03$ & $15.53 \pm 0.04$ & $15.08 \pm 0.03$ & $14.58 \pm 0.03$ & $15.41 \pm 0.02$ & $15.20 \pm 0.03$ \\
\hline 2006-04-15 & 2453841.1 & $15.87 \pm 0.04$ & $15.69 \pm 0.05$ & $15.14 \pm 0.04$ & $14.60 \pm 0.03$ & $15.47 \pm 0.03$ & $15.13 \pm 0.03$ \\
\hline 2006-04-17 & 2453842.7 & $16.23 \pm 0.04$ & $16.04 \pm 0.04$ & $15.35 \pm 0.04$ & $14.64 \pm 0.03$ & $15.42 \pm 0.02$ & $15.14 \pm 0.03$ \\
\hline 2006-04-17 & 2453842.9 & $16.30 \pm 0.05$ & $16.22 \pm 0.05$ & $15.40 \pm 0.05$ & $14.66 \pm 0.04$ & $15.41 \pm 0.04$ & $15.19 \pm 0.05$ \\
\hline 2006-04-17 & 2453843.3 & $16.37 \pm 0.04$ & $\cdots$ & $15.42 \pm 0.04$ & $14.67 \pm 0.03$ & $15.41 \pm 0.03$ & $15.19 \pm 0.04$ \\
\hline 2006-04-20 & 2453846.1 & $17.01 \pm 0.04$ & $16.91 \pm 0.05$ & $15.91 \pm 0.04$ & $14.94 \pm 0.03$ & $15.50 \pm 0.03$ & $15.16 \pm 0.03$ \\
\hline 2006-04-22 & 2453847.6 & $17.30 \pm 0.05$ & $17.34 \pm 0.07$ & $16.17 \pm 0.04$ & $15.00 \pm 0.03$ & $15.56 \pm 0.02$ & $15.19 \pm 0.03$ \\
\hline 2006-04-24 & 2453849.6 & $17.73 \pm 0.08$ & $18.13 \pm 0.10$ & $16.65 \pm 0.05$ & $15.18 \pm 0.03$ & $15.60 \pm 0.03$ & $15.27 \pm 0.03$ \\
\hline $2006-04-26$ & 2453851.6 & $18.22 \pm 0.09$ & $>18.8$ & $17.07 \pm 0.07$ & $15.46 \pm 0.03$ & $15.65 \pm 0.03$ & $15.23 \pm 0.03$ \\
\hline $2006-04-28$ & 2453853.5 & $18.78 \pm 0.10$ & $>18.8$ & $17.54 \pm 0.07$ & $15.83 \pm 0.03$ & $15.76 \pm 0.02$ & $15.23 \pm 0.03$ \\
\hline 2006-05-01 & 2453857.0 & $>18.8$ & $>18.8$ & $18.29 \pm 0.07$ & $16.31 \pm 0.03$ & $15.91 \pm 0.02$ & $15.22 \pm 0.02$ \\
\hline 2006-05-04 & 2453859.7 & $\ldots$ & $\ldots$ & $18.48 \pm 0.11$ & $16.64 \pm 0.04$ & $15.98 \pm 0.02$ & $15.22 \pm 0.03$ \\
\hline 2006-05-16 & 2453871.8 & $\ldots$ & $\cdots$ & $>18.6$ & $17.43 \pm 0.07$ & $\ldots$ & $15.38 \pm 0.02$ \\
\hline $2006-05-26$ & 2453882.0 & $\ldots$ & $\ldots$ & $>18.6$ & $17.91 \pm 0.09$ & $16.64 \pm 0.04$ & $15.50 \pm 0.03$ \\
\hline $2006-05-30$ & 2453885.9 & $\cdots$ & $\cdots$ & $>18.6$ & $>18.2$ & $16.72 \pm 0.02$ & $15.56 \pm 0.02$ \\
\hline
\end{tabular}

comoving frame, subject to the constraints of radiative and statistical equilibrium (Hillier \& Miller 1998). The ejecta are assumed to be spherically symmetric and chemically homogeneous, a good approximation given the very spatially confined regions of continuum and line formation (Dessart \& Hillier 2005a), and low polarization observed in the ejecta of photospheric phase Type II SN ejecta (Leonard \& Filippenko 2001). The expansion becomes truly homologous about a week after the explosion, but, for simplicity, is assumed homologous at all times. We adopt a density distribution characterized by a base density $\rho_{0}$ (at the base radius $R_{0}$ ) and a density exponent $n$, with $\rho(R)=\rho_{0}\left(R_{0} / R\right)^{n}$. The value of $\rho_{0}$ is chosen so that the Rosseland-mean optical depth $\tau_{\text {Rosseland }}$ at $R_{0}$ is $\sim 50$. The hydrogen-rich ejecta are always ionized at least once at the base and electron scattering is the primary source of continuum opacity, while the large $\mathrm{H}$ I bound-free cross sections ensure the thermalization of the radiation below the photosphere (see $\S 4.2$ and Figs 7-8-9 in Dessart \& Hillier 2005b). The value of $n$ is usually quite large, i.e., $\sim 10$, but could be much larger at early times, and can be constrained from line profile shapes, through the magnitude of the blueshift of peak emission of optical P-Cygni profiles (Dessart \& Hillier 2005a; Blondin et al. 2006; Aldering et al. 2005), or through the strength of P-Cygni profiles with respect to the continuum. For example, lower values of $n$ lead to P-Cygni profiles having stronger and broader absorption and emission components, and with a peak emission that is closer to line center. The early-time spectra of SNe 2005cs and 2006bp, show weak P-Cygni profiles with a strongly blueshifted peak emission, and point towards high values of $n$. We quote properties of models at the photosphere, which corresponds to the location where the continuum optical depth, integrated inwards from the outer grid radius, is $2 / 3$.

The luminosity at depth is not known in advance unless one uses a consistent hydrodynamical input of the ejecta properties, following simultaneously the timedependent evolution of the radiation field, internal en- 


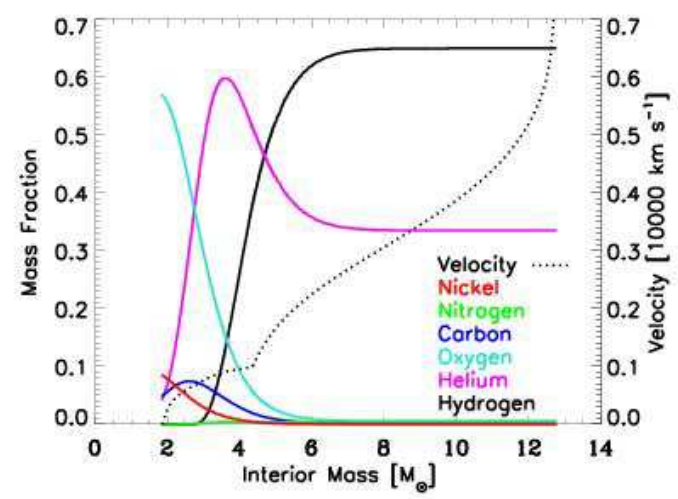

FIG. 2.- Illustration of the elemental mass fraction distribution versus interior mass for the most abundant species in a "reference" $15 \mathrm{M}_{\odot}$ model from Woosley \& Heger $\left(2007\right.$; a $1.83 \mathrm{M}_{\odot}$ neutron star is left behind in this explosion). The color coding differentiates species: Hydrogen (black), helium (violet), carbon (blue), oxygen (turquoise), nitrogen (green), and nickel (red). We also overplot the velocity (dotted black line), in units of $10,000 \mathrm{~km} \mathrm{~s}^{-1}$, to permit the inference of the elemental stratification in velocity space. The surface mass fractions are $X_{\mathrm{H}}=0.65, X_{\mathrm{He}}=0.34, X_{\mathrm{C}}=0.0014$, $X_{\mathrm{N}}=0.003, X_{\mathrm{O}}=0.005$, and with the adopted mixing, $X_{56 \mathrm{Ni}}=1.1$ $\times 10^{-9}$. [See the electronic edition of the Journal for a color version of this figure, and see $\$ 3$ for discussion.]

ergy, and level populations. Instead, we adjust the base (comoving-frame) luminosity so that the synthetic emergent (observer-frame) luminosity matches approximately the observed luminosity at each epoch, for an adopted distance and reddening. Usually, with our inferred distance and reddening, the emergent reddened synthetic flux is within a factor of 3 of the observed flux. We find that radial/luminosity scaling of that magnitude, preserving the ratio $L / R^{2}$ produce the same color and relative line strengths, line to continuum flux ratios, and other essential features of the spectrum. (see Dessart \& Hillier 2005a for a discussion).

Reddening is constrained using the Cardelli et al. (1988) law for both $\mathrm{SNe}$, assuming $R=3.1$. We adopt a single value of $E(B-V)$ to obtain satisfactory fits to the observed spectra at all epochs. This works best when the relative flux calibration is accurate. For SN $2005 \mathrm{cs}$, we find that the reddening is very small, i.e., $E(B-V) \sim 0.04$ (Baron et al. 2007 infer a value of 0.035 from a quantitative spectroscopic analysis with PHOENIX; Hauschildt \& Baron 1999), while for SN $2006 \mathrm{bp}$, it is quite large, i.e., $E(B-V) \sim 0.4$ (Ho et al. 1997 infer a value of 0.4 to the galaxy host NGC 3953 from the $\mathrm{H} \alpha / \mathrm{H} \beta$ ratio and assuming Case B recombination). Swift UVOT observations allow more leverage on the estimate of reddening than is permitted by optical observations alone by lengthening blueward the observed wavelength range.

To guide our reasoning when interpreting Type II SN spectra, in Fig. 2, we illustrate the chemical stratification and velocity distribution versus interior mass for a stellar model that is characteristic of the ejecta of Type II-P SNe, resulting from the explosion of a red supergiant (RSG) progenitor. The figure shows the ejecta of a $15 \mathrm{M}_{\odot}$ (on the main sequence) progenitor star model (ignoring rotation), exploded with a kinetic energy of $\sim 1 \mathrm{~B}\left(\equiv 10^{51} \mathrm{erg}\right)$ and with modest (artificial) nickel mix- ing (Woosley \& Heger 2007) .

At core collapse, this star has a mass of $\sim 13 \mathrm{M}_{\odot}\left(\sim 2 \mathrm{M}_{\odot}\right.$ have been lost through a wind during the pre-SN evolution), $1.83 \mathrm{M}_{\odot}$ is left behind in a protoneutron star and the rest escapes to form the $\mathrm{SN}$ ejecta. Starting from the surface, there is $\sim 7 \mathrm{M}_{\odot}$ of essentially homogeneous material, made up primarily of hydrogen $\left(X_{\mathrm{H}}=0.65\right)$ and helium $\left(X_{\mathrm{He}}=0.34\right)$ - just slightly evolved from the primordial, solar metallicity, mixture (Grevesse \& Sauval 1998). The velocity at the base of this hydrogen shell is about $2000 \mathrm{~km} \mathrm{~s}^{-1}$. Just below is a $\sim 3 \mathrm{M}_{\odot}$ shell dominated by helium $\left(X_{\mathrm{He}} \sim 0.6\right)$ and oxygen $\left(X_{\mathrm{O}} \sim 0.15\right)$, between velocities 1000 to $2000 \mathrm{~km} \mathrm{~s}^{-1}$. Finally, just above the inner mass cut at $1.83 \mathrm{M}_{\odot}$ resides an oxygen-rich shell $\left(X_{\mathrm{O}} \sim 0.6\right)$, with a $~ 0.1$ mass fraction of helium, carbon, and nickel. The radioactive nickel is deep inside, at velocities below $2000 \mathrm{~km} \mathrm{~s}^{-1}$, and separated by $\sim 10 \mathrm{M}_{\odot}$ of material from the photosphere at breakout.

In such (1D) radiation-hydrodynamics simulations, explosion parameters are adjusted to obtain the right amount of nickel production and mixing, which influence the light curve shape or the potential appearance of lines from radioactive isotopes at the photosphere (from ${ }^{56} \mathrm{Co}$, for example). For the peculiar Type II SN 1987A, this required allowing mixing out to $\sim 4000 \mathrm{~km} \mathrm{~s}^{-1}$ (Pinto \& Woosley 1988; Kumagai et al. 1989; Blinnikov et al. 2000), while for the Type II-P SN 1999em, nickel mixing out to $\sim 650 \mathrm{~km} \mathrm{~s}^{-1}$ seems optimal (Utrobin 2007). More detailed 2D hydrodynamical simulations of corecollapse supernovae explosions, assuming the energy demands are fulfilled, do not naturally predict nickel at large velocities, placing it at $\sim 2500 \mathrm{~km} \mathrm{~s}^{-1}$ (Hachisu et al. 1990, 1991; Kifonidis et al. 2000, 2003). Observationally, there is a range of inferred values and a conservative assumption for Type II-P SNe may be $\sim 1000$ $2000 \mathrm{~km} \mathrm{~s}^{-1}$, with $4000 \mathrm{~km} \mathrm{~s}^{-1}$ representing an extreme circumstance. In the above $15 \mathrm{M}_{\odot}$ model, ${ }^{56} \mathrm{Ni}$ is buried under about $10 \mathrm{M}_{\odot}$ of material, mostly composed of hydrogen and helium, so that any non-thermal excitation and ionization of the photosphere by radioactive isotopes is delayed until the end of the Plateau phase, when the photosphere has receded to these deep and slow-moving ejecta layers. In our analysis of the early photospheric phase of Type II-P SN, we neglect any contribution from radioactive decay. We expect slow and small variations in chemistry during the plateau phase, and, thus, adopt chemical homogeneity at a given epoch. This is consistent with our finding that $\mathrm{C}$ and $\mathrm{O}$ are only modestly enhanced at the photosphere one month after explosion. Finally, in such explosions, the density profile follows closely a power law distribution with exponent $\sim 10$ at depth in the hydrogen envelope, but is characterized by a much steeper drop-off near the surface layers, that may result from the strong radiation pressure in these layers at shock breakout (Blinnikov et al. 2000, Shigeyama \& Nomoto 1990). Such a steepening is not well-modeled by Lagrangean simulations, in particular for the shockbreakout phase, but our interpretation of our earliest observations supports this trend.

We adopt the surface composition of blue super-

${ }^{9}$ For the CMFGEN spectroscopic modeling that we present here, we do not use this ejecta structure at any time. 
TABLE 6

Model Characteristics For SN2005CS.

\begin{tabular}{|c|c|c|c|c|c|c|c|c|c|}
\hline $\mathrm{JD}$ & Day & $\begin{array}{l}\Delta t^{a} \\
\text { days }\end{array}$ & $\begin{array}{r}L_{\mathrm{CMF}, R_{0}} \\
\left(10^{8}\right.\end{array}$ & $\begin{array}{l}L_{\mathrm{OBS}, R_{\mathrm{Max}}} \\
\left.L_{\odot}\right)\end{array}$ & $\begin{array}{c}T_{\text {phot }} \\
(\mathrm{K})\end{array}$ & $\begin{array}{c}R_{\text {phot }} \\
\left(10^{14} \mathrm{~cm}\right)\end{array}$ & $\begin{array}{c}v_{\mathrm{phot}} \\
\left(\mathrm{km} \mathrm{s}^{-1}\right)\end{array}$ & $\begin{array}{c}\rho_{\text {phot }} \\
\left(10^{-14} \mathrm{~g} \mathrm{~cm}^{-3}\right)\end{array}$ & $n$ \\
\hline 2453552.25 & 2005-06-30 & 1.85 & 3.0 & 2.69 & 15750 & 2.00 & 6880 & 24.6 & 20 \\
\hline 2453553.25 & $2005-07-01$ & 2.85 & 2.3 & 1.99 & 13350 & 2.55 & 6950 & 10.8 & 12 \\
\hline 2453554.50 & 2005-07-02 & 4.10 & 2.3 & 2.01 & 13420 & 2.54 & 6370 & 10.6 & 12 \\
\hline 2453555.25 & 2005-07-03 & 4.85 & 2.0 & 1.74 & 10850 & 3.26 & 6080 & 7.6 & 10 \\
\hline 2453556.00 & 2005-07-04 & 5.60 & 1.8 & 1.64 & 9300 & 3.91 & 5450 & 6.8 & 10 \\
\hline 2453557.75 & 2005-07-05 & 7.35 & 1.8 & 1.56 & 8620 & 4.93 & 4780 & 5.6 & 10 \\
\hline 2453558.25 & 2005-07-06 & 7.85 & 1.5 & 0.97 & 8250 & 4.18 & 5230 & 6.0 & 10 \\
\hline 2453561.25 & 2005-07-09 & 10.85 & 1.5 & 1.21 & 7770 & 5.97 & 4710 & 4.7 & 10 \\
\hline 2453562.50 & $2005-07-10$ & 12.10 & 1.5 & 1.19 & 7120 & 6.38 & 4580 & 6.0 & 10 \\
\hline 2453563.50 & $2005-07-11$ & 13.10 & 1.5 & 1.10 & 7300 & 5.88 & 4440 & 8.9 & 10 \\
\hline 2453564.25 & $2005-07-12$ & 13.85 & 1.5 & 1.08 & 6980 & 6.01 & 4320 & 10.9 & 10 \\
\hline 2453566.00 & $2005-07-14$ & 15.60 & 1.1 & 0.79 & 6700 & 5.47 & 3930 & 28.2 & 10 \\
\hline 2453580.25 & $2005-07-28$ & 29.85 & 1.0 & 1.17 & 6140 & 7.84 & 2240 & 125.6 & 10 \\
\hline
\end{tabular}

Note. - For each date in our sample of observations, we provide the following CMFGEN model parameters: Base comovingframe luminosity $L_{\mathrm{CMF}, R_{0}}$ and emergent observer-frame luminosity $L_{\mathrm{OBS}} R_{\mathrm{Max}}$ (in $\mathrm{L}_{\odot}$; note that for the model on the last date, the observer-frame luminosity has not fully relaxed to the base condition, a problem that stems from the presence of a steep and hard-to-model recombination front at the photosphere), photospheric conditions describing the electron temperature $T_{\text {phot }}$ (in K), the radius $R_{\text {phot }}\left(\right.$ in $10^{14} \mathrm{~cm}$ ), the velocity $v_{\text {phot }}\left(\right.$ in $\mathrm{km} \mathrm{s}^{-1}$ ), and the density (in $10^{-14} \mathrm{~g} \mathrm{~cm}^{-3}$ ), together with the density exponent $n$ characterizing the density law $\rho(r)=\rho_{\text {phot }}\left(R_{\text {phot }} / r\right)^{n}$. (See 3 for a presentation of the modeling approach). ${ }^{a}: \Delta t$ is the time elapsed since discovery.

giants analyzed by Crowther et al. (2006), which is in very close agreement with the outer composition of the $15 \mathrm{M}_{\odot}$ model described above. By number, we adopt $\mathrm{H} / \mathrm{He}=5, \mathrm{C} / \mathrm{He}=0.0004, \mathrm{~N} / \mathrm{He}=0.0013, \mathrm{O} / \mathrm{He}$ $=0.0016$, with all metals taken at their solar value (Grevesse \& Sauval 1998). Given as mass fractions, these abundances are: $X_{\mathrm{H}}=0.55, X_{\mathrm{He}}=0.44, X_{\mathrm{C}}=0.0005$, $X_{\mathrm{N}}=0.002, X_{\mathrm{O}}=0.003$, and $X_{\mathrm{Fe}}=0.0013$. At later times, when in particular O I $7770 \AA$ gains in strength, we need to enhance both the carbon and the oxygen abundances, with $\mathrm{C} / \mathrm{He}=0.001, \mathrm{O} / \mathrm{He}=0.01$, and to reduce the nitrogen abundance, $\mathrm{N} / \mathrm{He}=0.001$ to match the data.

A key asset of CMFGEN is the explicit treatment of line blanketing using a detailed description of the atomic structure for a large number of species. The iterative march to solving many non-LTE level populations together with the radiation field comes at great CPU expense. We optimize the problem by including only the relevant ions of the most important species, although the ejecta ionization stratification still requires that at least three ionization stages of iron, for example, be included to capture the sources of bound-bound and bound-free opacity at all depths. Typically, any model crafted for very early conditions ( to match the first few, nearly featureless spectra of SNe 2005cs and 2006bp, with weak He I $5875 \AA$ ) will contain the following species and levels (the suffixed parenthesis contains the number of fulland super-levels; see Hillier \& Miller 1998 for details): H I $(30,20)$, He I $(51,40)$, He II $(30,13)$, C II $(26,14)$, C III $(112$, 62), C IV $(64,59), \mathrm{N}$ II $(3,3)$, N III $(26,26), \mathrm{N}$ IV $(60,34)$, N v $(67,45)$, O II $(3,3)$, O III $(115,79)$, O IV $(72,53)$, O V $(152,73)$, Si IV $(48,37)$, Si v $(71,33)$, Fe II $(309,116)$, Fe III $(563,191)$, Fe IV $(787,120)$, Fe V $(191,47)$, Fe VI $(433,44)$, Ni III $(67,15)$, and Ni IV $(242,36)$. At one week after explosion, when He I $5875 \AA$ is strong, we take out N IV, O v, Si IV, Siv, Fe VI, and Ni IV, and add Ni $(104,54)$, O I (75,23), Mg II $(65,22)$, Ca II $(77,21)$, Si II $(59,31)$, Ti II $(152,37)$, Ti III $(206,33)$, Ni II $(93,19)$, and Ni III $(67,15)$. When hydrogen starts recombining, we put more emphasis on low ionization states and adopt the following model atoms: H I $(30,20)$, He I $(51,40), \mathrm{C}_{\mathrm{I}}(63,33), \mathrm{C}$ II $(59,32)$, N I (104,54), Na I (71,22), Mg II (65,22), Si II (59, 31), O I $(75,23)$, Ca II $(77,21)$, Fe I $(136,44)$, Fe II $(309,116)$, Fe III $(477,61)$, Ni II $(93,19)$, and Ti II $(152,37)$. References to the corresponding atomic data sources are given in Dessart \& Hillier (2005a).

To summarize, inputs to a CMFGEN model are global characteristics of the ejecta such as base (comovingframe) luminosity, radius, chemical composition, velocity and density. These parameters are iterated (see Dessart \& Hillier 2005a for details) until the synthetic spectrum matches the observations. Assessment of suitability is done by eye. Spectroscopic observations used here are accurate in relative flux, while only accurate to within 10-20\% in absolute flux. For spectral fitting, relative flux calibration is essential while absolute flux calibration is not. When inferring distances, we use the photometry to set the absolute flux scale. One important result of our study is that the CMFGEN model parameters needed to fit the optical data of SNe 2005cs and 2006bp during the first 2-3 weeks after explosion also naturally explain the Swift UVOT data simultaneously. There appears to be a common, photospheric, origin for the UV and optical light for both objects: circumstellar interaction contributes very little to the observed light in these spectral regions (see also Brown et al. 2007). Note that SN 2006bp was, however, detected in the X-rays for up to 12 days after explosion, suggesting that interaction between the ejecta and the circumstellar medium (CSM) does occur (Immler et al. 2007), but without any sizable effect on the UV and the optical flux.

The first month after explosion, when the photosphere resides in the ionized hydrogen-rich layers of the ejecta, is not the best epoch to probe the innermost details of the explosion mechanism. However, it is ideal for distance determinations based on variants of the Expanding Photosphere Method: A true continuum exists and the effects of line-blanketing are weak in the optical; the properties of the ejecta are well determined by the model, using both the continuum energy distribution and lines from the most abundant ions. The homogeneous 


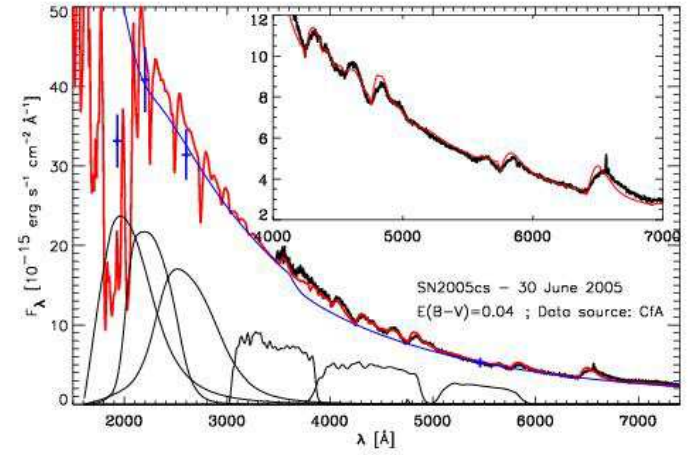

FIG. 3.- Comparison between the reddened $(\mathrm{E}(\mathrm{B}-\mathrm{V})=0.04)$ synthetic spectrum (red:full; blue: continuum only; $T_{\text {phot }}=15750 \mathrm{~K}$ ) and the observations of SN 2005cs on the 30th of June, 2005, including CfA observations (black) and Swift photometry (blue crosses). We insert a panel that zooms on the optical range to allow a better assessment of the fit quality. For completeness, we overlay the Swift UVOT filter passbands in black, (scaled for visibility, roughly in proportion to the observed flux), with, from short to long wavelengths, uvw $2\left(\lambda_{\mathrm{c}}=2030 \AA\right)$, uvm $2\left(\lambda_{\mathrm{c}}=2231 \AA\right)$, uvw 1 $\left(\lambda_{\mathrm{c}}=2634 \AA\right), u\left(\lambda_{\mathrm{c}}=3501 \AA\right), b\left(\lambda_{\mathrm{c}}=4329 \AA\right), v\left(\lambda_{\mathrm{c}}=5402 \AA\right)$, where $\lambda_{c}$ is their effective wavelength assuming a Vega-like spectrum (Poole et al. 2007). [See the electronic edition of the Journal for a color version of this figure, and see 4.1 for discussion.]

hydrogen-rich envelope ensures that a well defined photosphere exists and that radiation thermalizes at depth, allowing the adequate use of the diffusion approximation at the model base. There, our non-LTE approach naturally predicts LTE conditions, the departure coefficients of all levels tending to unity, and at the thermalization depth, the mean intensity is well represented by a blackbody (LTE is, in other words, never enforced). The analysis presented here allows the inference of distances to Type II-P SNe (see 6.1 and 6.2 ).

To be more concise, we present all model parameters in Table 6 and 7, but mention in the text only the parameters that are most directly related to the spectroscopic evolution, i.e., $v_{\text {phot }}, T_{\text {phot }}$, and $n$.

\section{SN $2005 \mathrm{cs}$}

SN 2005cs was discovered in the Whirlpool galaxy (M 51a; NGC 5194) by Kloehr et al. (2005) on 28.905 June 2005 (JD 2453550.4). It was not detected on 26.89 June below the limits $B \geq 17.3, V \geq 17.7$, and $R \geq 17.6$ (P06), which places a narrow window of two days on the explosion date. In this work, we employ observations from various sources at 13 epochs, starting on 30 June 2005, which could be within 3 days of the explosion. Note that M 51a, an SA(s)bc galaxy, also hosted the Type Ic SN 1994I (Wheeler et al. 1994; Filippenko et al. 1995; Baron et al. 1996). Importantly, Maund et al. (2005) and $\mathrm{Li}$ et al. (2006) associate the SN 2005 cs event with a $9_{-2}^{+3} \mathrm{M}_{\odot} \mathrm{K} 0-\mathrm{M} 4 \mathrm{RSG}$ progenitor star, based on the analysis of pre-explosion HST images, compatible with the observed plateau evolution of the light curve in the optical bands. SN 2005cs was not detected in the X-rays and the upper limit to the X-ray luminosity yields a limit to the progenitor mass loss rate of $\dot{\mathrm{M}} \lesssim 1 \times 10^{-5} \mathrm{M}_{\odot} \mathrm{yr}^{-1}\left(v_{\mathrm{w}} / 10\right) \mathrm{km} \mathrm{s}^{-1}$, where $v_{\mathrm{w}}$ is the asymptotic velocity of the progenitor wind (Brown et al. 2007). Model properties are given in Table 6] and corresponding synthetic spectral fits to observations are presented in Figs. 3 . 7

\subsection{The early times: 30th of June until 4th of July 2005}

We show the first observations (blues crosses: Swift UVOT photometry; black curve: optical spectrum of 30 June 2005 from CfA) of SN 2005cs in Fig. 3, together with synthetic spectra (red: full; blue: continuum only), reddened with $E(B-V)=0.04$ and scaled by a factor 1.17. Observations reveal a very hot spectrum, with a steep decline of the observed flux longward of $\sim 2300 \AA$. We identify optical lines of the Balmer series $(\mathrm{H} \alpha, \mathrm{H} \beta$, and $\mathrm{H} \gamma$ ), He I $5875 \AA$, the N II multiplets at $\sim 4600 \AA$ and $\sim 5400 \AA$, and, as suggested by Baron et al. (2007), an O II multiplet at $\sim 4600 \AA$. Note that the lower ionization inferred for the earliest spectra of SN 1999em suggested that N II alone was sufficient to reproduce adequately the observed features at both $4600 \AA$ and $5400 \AA$. It is the higher ionization in the first spectrum of SN 2005cs that calls for this novel O II identification, more consistent with solar CNO chemistry, and slightly different from the abundances used in DH06 (see our discussion in $\widehat{3} 3$ and 97 ). The $4300-5000 \AA$ region also contains numerous He I lines. The observed dip shortward of $2000 \AA$ stems from Fe III line-blanketing, rather than dust extinction (the continuum-only synthetic spectrum, shown in blue, does not show this dip). The individual contributions from all important ions on that date are presented in Fig. 5, left panel, of Brown et al. (2007). On that date, we have $T_{\text {phot }}=15750 \mathrm{~K}, v_{\text {phot }}=6880 \mathrm{~km} \mathrm{~s}^{-1}$, and $n=$ 20. Such a steep density distribution is required to bring optical lines down to their (weak) observed strength, by reducing their emission volumes. This is higher than the density exponent adopted in the past, but these are also the first analyses of such early-time observations of Type II-P SN spectra. A steep density gradient is also used to fit the earliest observations of SN 2006bp. We discuss this choice and comment on its implications in $\$ 7.1$.

We present P06's observations of SN 2005cs on the 1st July 2005 in Fig. 4, top left panel. The slope of the spectrum in the blue appears steeper than predicted by the $S$ wift UVOT data points, which are quite closely matched by our model (the observed flux in the spectrum, inaccurate below $\sim 4000 \AA$, is omitted; Pastorello, priv. comm.). The synthetic flux is scaled by a factor 1.1. The SN ejecta is getting cooler $\left(T_{\text {phot }}=13350 \mathrm{~K}\right)$ at the photosphere, but with a comparable velocity (within the uncertainties; $\left.v_{\text {phot }}=6950 \mathrm{~km} \mathrm{~s}^{-1}\right)$. The density exponent $n$ has been reduced to a value of 12 (the density distribution flattens), motivated by the strengthening of optical line features, whose identity is the same as that on 30 June. The UV flux is weaker, resulting primarily from the cooling of the ejecta - the line blanketing is still mostly due to Fe III.

Little changes as we move onto the 2nd of July 2005, as shown in Fig. 4, top right panel, with a model characterized by $T_{\text {phot }}=13420 \mathrm{~K}, v_{\text {phot }}=6370 \mathrm{~km} \mathrm{~s}^{-1}$, and $n=12$, or a lower ejecta velocity (Dessart \& Hillier 2005a). This $10 \%$ range in expansion velocity $\left(\sim 500 \mathrm{~km} \mathrm{~s}^{-1}\right)$ may in fact be physical, reflecting the potential presence of fluid instabilities or turbulence. The Swift UVOT photometry would be better fit by a faster decline in the UV than our CMFGEN model provides. This situation improves on July 3rd, the following day 

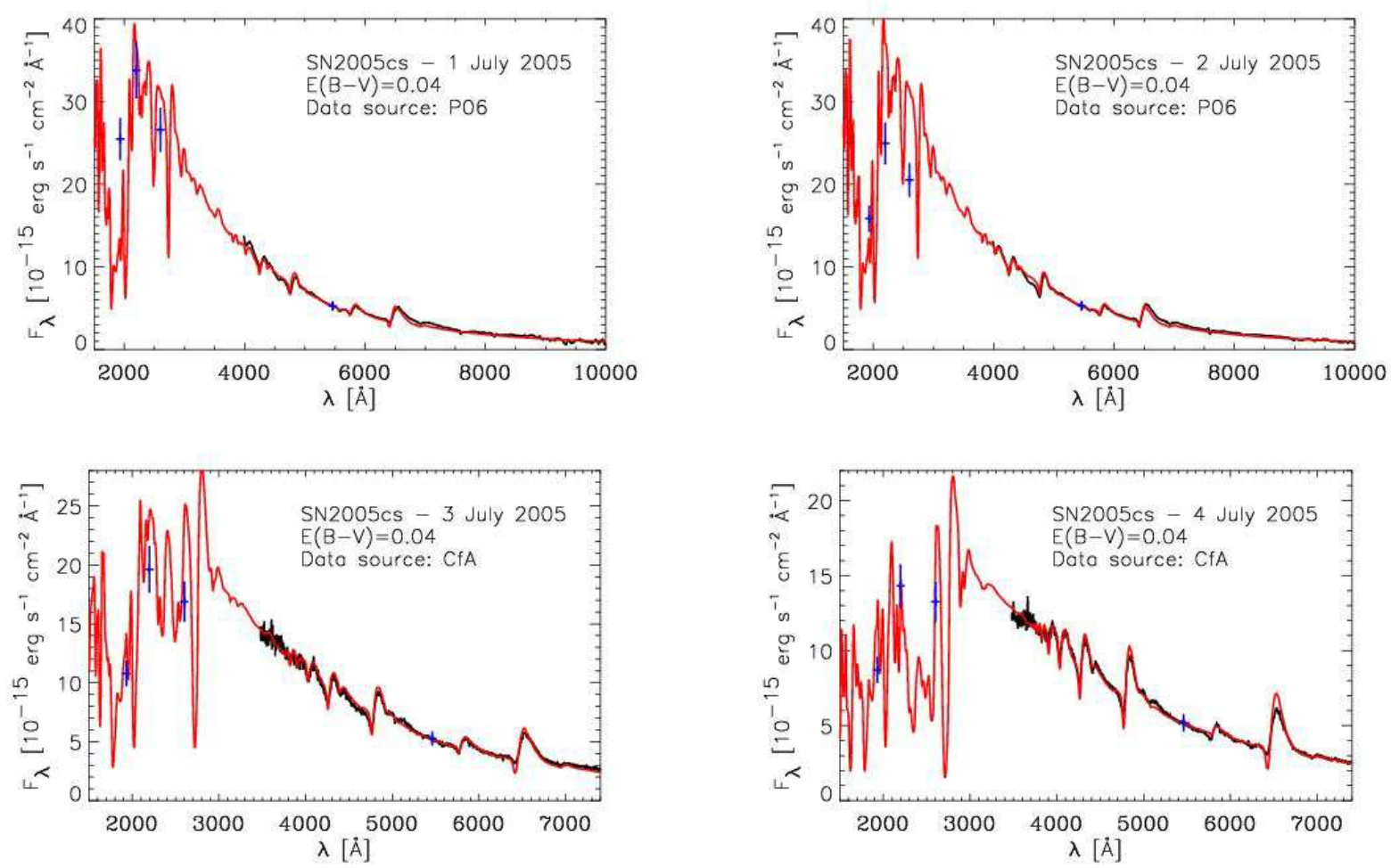

FIG. 4. - Same as Fig. 3 but for the SN 2005cs observations of July 1st (top left; $\left.T_{\text {phot }}=13350 \mathrm{~K}\right), 2$ nd (top right; $T_{\text {phot }}=13420 \mathrm{~K}$ ), 3rd (bottom left; $T_{\mathrm{phot}}=10850 \mathrm{~K}$ ), and 4 th (bottom right; $T_{\text {phot }}=9300 \mathrm{~K}$ ), 2005 . A full set of model parameters is given in Table 6 [See the electronic edition of the Journal for a color version of this figure, and see 4.1 for discussion.]

(Fig. 4, bottom left panel), where a single model fits both the Swift UVOT photometry and the optical spectrum, with parameters $T_{\text {phot }}=10850 \mathrm{~K}, v_{\text {phot }}=6080 \mathrm{~km} \mathrm{~s}^{-1}$, and $n=10$. The photosphere is both cooler and slower, while the density exponent is now set to 10 at all remaining epochs. The observations on July 4 th resemble those of July 3rd in the optical, but the UVOT photometry indicates a fading of the UV flux. In the bottom right panel of Fig. 4, we show the model fit to the July 4th observations is cooler, with $T_{\text {phot }}=9300 \mathrm{~K}, v_{\text {phot }}=5450 \mathrm{~km} \mathrm{~s}^{-1}$, and $n=10$.

These early observations show a strong UV flux that dominates over the optical flux, with low extinction. Optical lines are $\mathrm{HI}$, He I, O II and N II, with blanketing in the UV from Fe III. The density distribution is steep, flattening over a few days to the canonical $n \sim 10$ exponent predicted for blast wave solutions (Imshennik \& Nadezhin 1988; Shigeyama \& Nomoto 1990, Chevalier 1982; Ensman \& Burrows 1992, Blinnikov et al. 2000). We surmise that the steep density profile may reflect work done by the intense UV radiation at and shortlyafter shock breakout (see \$7.1). Our models support a common origin for both the UV and the optical light observed, with emission from the regions at and above the photosphere. No additional contribution to the UV and optical fluxes is present from an external source, such as an interaction between the SN ejecta and the CSM.

\subsection{The intermediate times: 5th of July until 14th of July 2005}

In this epoch, the flux level decreases dramatically in the UV and metal lines, mostly of Fe II, appear in the optical. This is the onset of the hydrogen-recombination epoch leading to the "Plateau" phase of such Type II SNe. At the same time, He I $5875 \AA$ weaken, so that the feature at $\sim 5900 \AA$ is due only to Na I D at late times.

These spectral properties are visible in Fig. 5, top left panel, in which observations on the 5th of July are fitted with a model characterized by $T_{\text {phot }}=8620 \mathrm{~K}$, $v_{\text {phot }}=4780 \mathrm{~km} \mathrm{~s}^{-1}$, and $n=10$. The O I $7770 \AA$ line is now clearly seen, following the cooling and the recession of the photosphere. The SN 2005cs ejecta have lower velocities than SN 1999em: this allows the clear identification of this line profile, which was severely corrupted by the atmospheric extinction in the spectra of SN 1999em (DH06). Similarly, while Si II $6355 \AA$ was predicted to overlap with $\mathrm{H} \alpha$ in the spectrum of SN 1999em (Figs. 3 and 5 in Dessart \& Hillier 2005a), we clearly observe it here as a separate line blueward of the $\mathrm{H} \alpha$ trough (see also P06). This line, in combination with unidentified N II/OII lines at similar Doppler velocities in the blue wing of $\mathrm{H} \beta$ and He I $5875 \AA$, led to speculations on the presence of inhomogeneities above the photosphere (Leonard et al 2002ab). At early epochs, it seems these subtle spectral features can be explained quite naturally by numerous lines of $\mathrm{O}$ II (see above), N II, HeI, and Si II. At later times, the presence of a cool dense shell at the outer edge of Type II-P SN ejecta is expected to produce real absorption dips in the trough of $\mathrm{H} \alpha$ (and He I $10830 \AA$; see Chugai et al. 2007). The fit to observations on July 6th is shown on the top right panel of Fig. 5. with little change from the previous day except for a modest decline in temperature (e.g., $T_{\text {phot }}=8250 \mathrm{~K}$ ).

By the 9th of July (Fig. 5, middle left panel), the UV flux is now an order of magnitude lower than the optical flux. The CaII H\&K doublet appears as a strong 

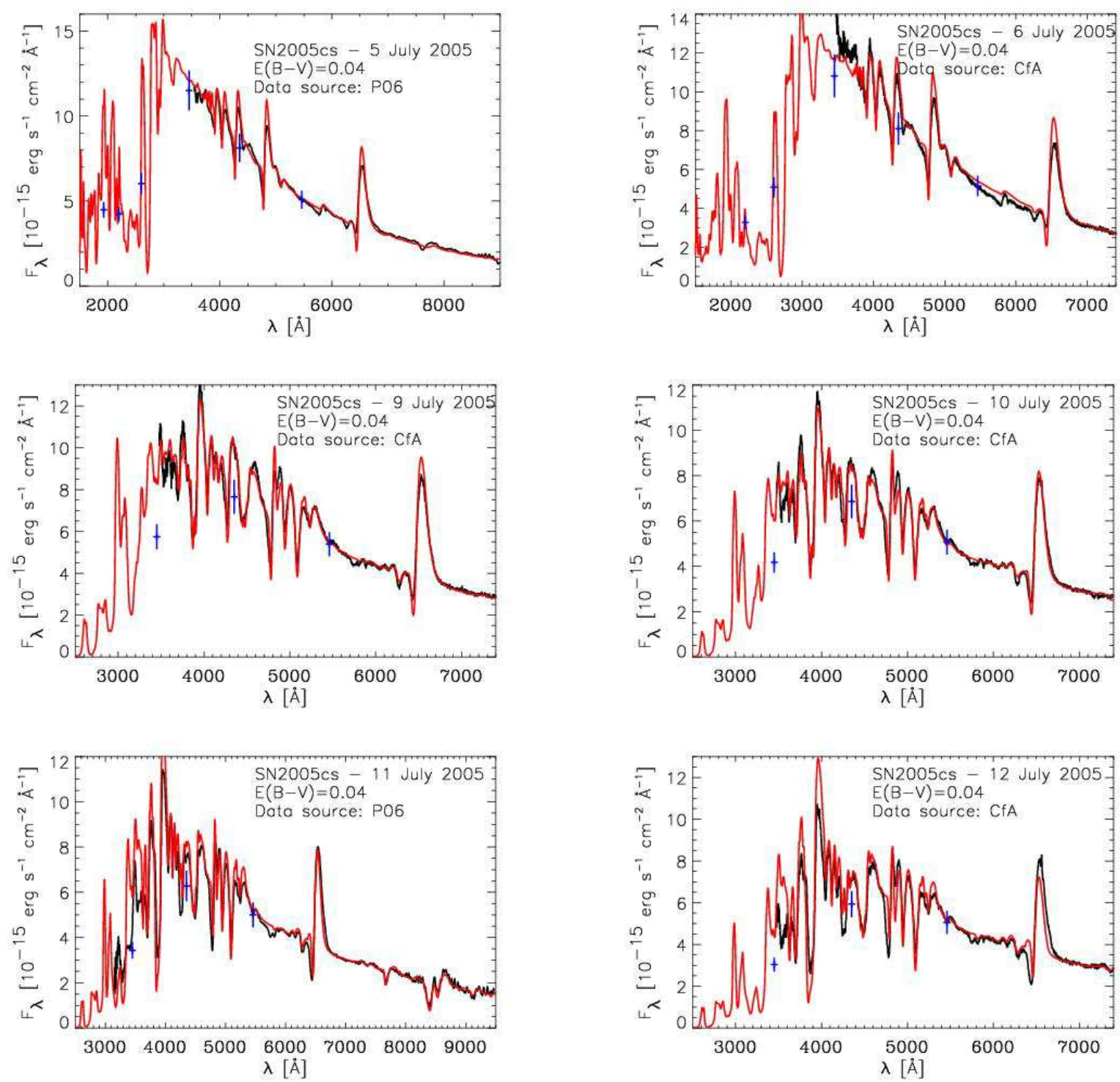

FIG. 5.- Same as Fig. 3 but for the SN 2005cs observations of July 5 th (top left; $T_{\text {phot }}=8620 \mathrm{~K}$ ), 6 th $\left(\right.$ top right; $\left.T_{\text {phot }}=8250 \mathrm{~K}\right)$, 9 th $\left(\right.$ middle left; $\left.T_{\text {phot }}=7770 \mathrm{~K}\right), 10$ th $\left(\right.$ middle right; $\left.T_{\text {phot }}=7120 \mathrm{~K}\right), 11$ th $\left(\right.$ bottom left; $\left.T_{\text {phot }}=7300 \mathrm{~K}\right), 12$ th $\left(\right.$ bottom right; $\left.T_{\mathrm{phot}}=6980 \mathrm{~K}\right)$, 2005. A full set of model parameters is given in Table 6. [See the electronic edition of the Journal for a color version of this figure, and see 4.2 for discussion.]
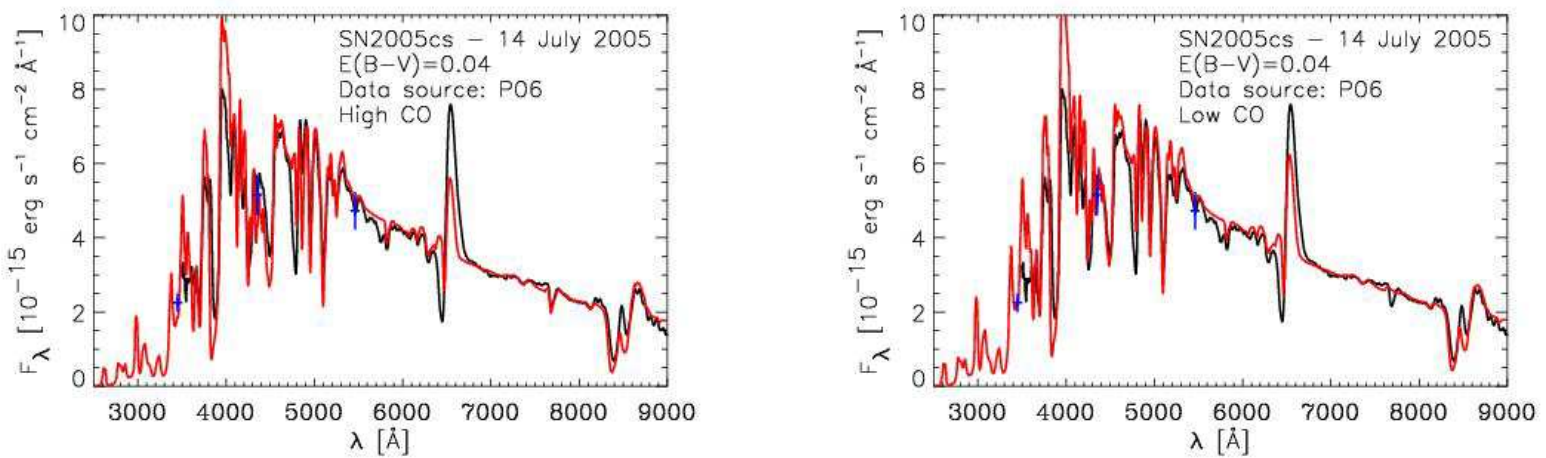

FIG. 6. - Same as Fig. 3 but this time for P06's observations of SN 2005cs on the 14th of July, 2005, and using a model with enhanced (left) or day-one (right) carbon and oxygen abundances. [See the electronic edition of the Journal for a color version of this figure, and see 4.2 for discussion.] 


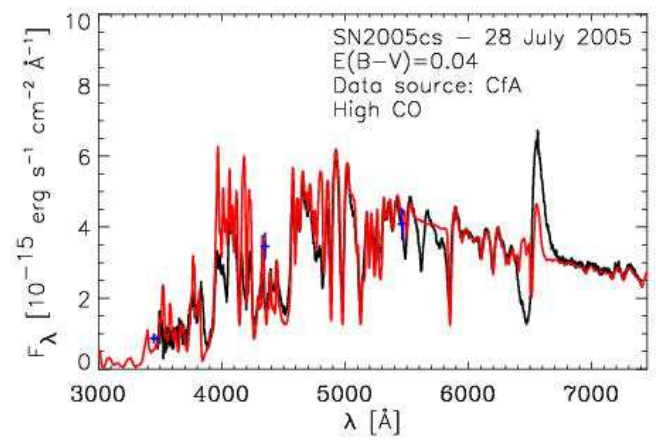

FIG. 7.- Same as Fig. 3 but this time for the observations of SN 2005cs on the 28th of July, 2005. [See the electronic edition of the Journal for a color version of this figure, and see 4.3 for discussion.]

P-Cygni line profile at the blue edge of the optical spectrum, strengthening with time, together with the Fe II lines present throughout the UV range (as a forest of overlapping lines that give an apparently continuous light blocking effect - see Fig. 5 in Dessart \& Hillier 2005a) and up to $\sim 5200 \AA$. Ni II (shortward of $2500 \AA$ ) and Ti II (in the $3000 \AA$ region) also contribute significant line blanketing. The model properties for the 9th of July are $T_{\text {phot }}=7770 \mathrm{~K}, v_{\text {phot }}=4710 \mathrm{~km} \mathrm{~s}^{-1}$, and $n=10$.

Subsequent evolution is quite slow from the 10th (Fig. 15 middle right panel), 11th (Fig. 5, bottom left panel), 12th (Fig. 5, bottom right panel), and 14th (Fig. 6). Over this 5-day period, the photospheric layers cool slowly and modestly $(7120 \mathrm{~K}, 7300 \mathrm{~K}, 6980 \mathrm{~K}$, $6700 \mathrm{~K}$, respectively), and slow down $\left(4580 \mathrm{~km} \mathrm{~s}^{-1}\right.$, $4440 \mathrm{~km} \mathrm{~s}^{-1}, 4320 \mathrm{~km} \mathrm{~s}^{-1}, 3930 \mathrm{~km} \mathrm{~s}^{-1}$ ). On the $14 \mathrm{th}$, the P06 spectrum extends beyond $9000 \AA$ and reveals the presence of the Ca II $8500 \AA$ multiplet, providing a good check that the ionization state of the SN ejecta is well modeled. Fitting the O I $7770 \AA$ line requires a higher oxygen abundance $(\mathrm{O} / \mathrm{He}=0.01)$, which we adopt for $\mathrm{SN}$ 2005 cs starting on the 11th of July. We show a comparison of the enhanced and standard oxygen abundance CMFGEN models in the left and right panels of Fig. 6, to show the effect on the predicted strength of the O I $7770 \AA$ line. Note finally that the $\mathrm{H} \alpha$ line strength (as well as $\mathrm{H} \beta$, although less discernible) is underestimated by a factor of two in both absorption and emission, the more so for higher carbon/oxygen abundances (corresponding to lower hydrogen mass fractions).

\subsection{July 2005}

The observation of the 28th of July 2005 is the last in our SN 2005cs sample. This is about one month after the first observation taken and well into the plateau phase. We show our fits to these observations in Fig. 17 for which the model parameters are $T_{\text {phot }}=6140 \mathrm{~K}$, $v_{\text {phot }}=2240 \mathrm{~km} \mathrm{~s}^{-1}$, and $n=10$. The photosphere has now receded to slow-moving ejecta layers close to the bottom of the hydrogen shell.

In practice, given the low luminosity and low velocity of this SN envelope, the (outer) hydrogen shell of the progenitor may stretch even further down to the slower velocity and more $\mathrm{C} / \mathrm{O}$ enriched layers. Unfortunately, the CfA observations do not cover the $\mathrm{O}$ I and $\mathrm{C}$ I lines longward of $7500 \AA$, which would provide a measure of the oxygen and carbon mass fractions. In DH06, and by comparison with the surface chemistry of the progenitor, we inferred significant enhancements in carbon and oxygen abundances in the photospheric layers of SN $1999 \mathrm{em}$ at 45 days after explosion. The main line diagnostics are O I $7775 \AA$ and O I $7984 \AA$, while redder transitions at $8446 \AA$ and $9264 \AA$ overlap with Ca II, Mg II, and $\mathrm{C}$ I lines, and are therefore less reliable. Useful C I diagnostics are the features at $\sim 9100 \AA, 9400 \AA$, and $9600 \AA$, due to transitions of the $(3 \mathrm{p}-3 \mathrm{~s})$ series at $9061 \AA, 9062 \AA$, $9078 \AA, 9088 \AA,, 9112 \AA, 9405 \AA, 9603 \AA, 9621 \AA$, and $9658 \AA$. In the near-IR, the $\mathrm{C}$ I $1.07 \mu \mathrm{m}$ is an additional diagnostic, although it overlaps with $\mathrm{P} \gamma$, and potentially with He I $1.083 \mu \mathrm{m}$. Observations in this wavelength range would be especially valuable.

Observing the $\mathrm{C} / \mathrm{O}$ abundance change would be important and warrants thorough late time spectroscopic observations in the photospheric phase of Type II SNe, extending at least up to $1 \mu \mathrm{m}$. A census of lines contributing in the optical is shown in Fig. 8 of DH06. Fe II blankets completely whatever UV flux emerges from the thermalization layers below the photosphere. Ti II and Fe II leave a complex set of features in the optical, most noticeably resulting in a pronounced dip in the B-band region. $\mathrm{Na}$ I D is reproduced here by enhancing its abundance by a factor of four above its solar metallicity value (Grevesse \& Sauval 1998). However, the predicted line remains narrower than observed and time-dependent effects were found to lead to a strong and broad line profile without any change in sodium abundance. The underestimate of the $\mathrm{H} \alpha$ line strength suggests that this is the epoch when our steady-state approach departs significantly from the more correctly-computed time-dependent conditions in the SN ejecta (Dessart \& Hillier 2007a,b).

\section{SN 2006bp}

SN 2006bp was discovered in NGC 3953, an SB(r)bc galaxy, by Koichi Itagaki (Teppo-cho, Yamagata, Japan) around 9.60 April 2006 (JD 2453835.1), and located at R.A. $=11 \mathrm{~h} 53 \mathrm{mn} 55 \mathrm{~s} .74$, Decl. $=+52^{\circ} 21^{\prime} 09 " .4$ (equinox 2000.0 ), which is 62 " east and 93" north of the center of NGC3953. Quimby et al. (2006) obtained an optical spectrum on March 11.11 UT with the 9.2-m HobbyEberly Telescope (HET), reporting the observation of a blue continuum, a narrow absorption at $592 \mathrm{~nm}$ (associated with $\mathrm{NaID}$ ), and line features at $583 \mathrm{~nm}$ (associated with He I $5875 \AA$ ), and $627 \mathrm{~nm}$ (associated with $\mathrm{H} \alpha$ ). SN 2006bp was not detected in unfiltered ROTSE-IIIb images taken on Apr. 9.15 (limiting mag about 16.9), but it was found at about $15.3 \mathrm{mag}$ on images taken on Apr. 10.15. SN 2006bp was caught promptly after the explosion, as confirmed from our analysis of the spectra. Immler et al. (2007) report the $4.5 \sigma$ detection of X-ray emission at the level of $1.8 \pm 0.4 \times 10^{39} \mathrm{erg} \mathrm{s}^{-1}$ up until 12 days after discovery, indicating circumstellar interaction with a pre-SN RSG wind characterized by $\dot{\mathrm{M}} \sim 10^{-5} \mathrm{M}_{\odot} \mathrm{yr}^{-1}$. This X-ray luminosity is less than $1 \%$ of the UV and optical luminosity in the combined UV and optical ranges- and differs from the strongly interacting Type IIn SNe ${ }^{10}$. Model properties are given

\footnotetext{
10 Type IIn spectra are characterized by narrow lines for all ions that contribute and most conspicuously $\mathrm{H}$ i and Fe II. Examples of such Type IIn SNe are 1994W (Chugai et al. 2004), 1995G (Pas-
} 
TABLE 7

Model Characteristics for SN2006bP.

\begin{tabular}{|c|c|c|c|c|c|c|c|c|c|}
\hline Julian Date & Day & $\begin{array}{l}\Delta t^{a} \\
\text { Days }\end{array}$ & $\begin{array}{r}L_{\mathrm{CMF}, R_{0}} \\
\quad\left(10^{8}\right.\end{array}$ & $\begin{array}{l}L_{\mathrm{OBS}, R_{\mathrm{Max}}} \\
\left.L_{\odot}\right)\end{array}$ & $\begin{array}{c}T_{\text {phot }} \\
(\mathrm{K})\end{array}$ & $\begin{array}{c}R_{\text {phot }} \\
\left(10^{14} \mathrm{~cm}\right)\end{array}$ & $\begin{array}{c}v_{\text {phot }} \\
\left(\mathrm{km} \mathrm{s}^{-1}\right)\end{array}$ & $\begin{array}{c}\rho_{\text {phot }} \\
\left(10^{-14} \mathrm{~g} \mathrm{~cm}^{-3}\right)\end{array}$ & $n$ \\
\hline 2453836.6 & 2006-04-11 & 1.5 & 15 & 16.3 & 20600 & 2.386 & 14100 & 51.7 & 50 \\
\hline 2453837.6 & 2006-04-12 & 2.5 & 15 & 15.1 & 18120 & 3.165 & 12550 & 31.6 & 50 \\
\hline 2453842.6 & 2006-04-17 & 7.5 & 9 & 6.9 & 11800 & 5.541 & 11300 & 8.3 & 20 \\
\hline 2453844.6 & 2006-04-19 & 9.5 & 9 & 6.6 & 11400 & 5.868 & 11050 & 6.5 & 16 \\
\hline 2453845.8 & 2006-04-20 & 10.7 & 9 & 6.2 & 10700 & 6.485 & 10300 & 4.5 & 12 \\
\hline 2453846.7 & 2006-04-21 & 11.6 & 9 & 6.2 & 10700 & 6.485 & 10300 & 4.5 & 12 \\
\hline 2453849.6 & 2006-04-24 & 14.5 & 5 & 3.6 & 9350 & 6.345 & 7920 & 4.2 & 10 \\
\hline 2453850.6 & 2006-04-25 & 15.5 & 2.7 & 1.8 & 8150 & 6.580 & 8750 & 4.0 & 10 \\
\hline 2453855.7 & 2006-04-30 & 20.6 & 1.5 & 1.1 & 7320 & 6.000 & 6450 & 7.3 & 10 \\
\hline 2453856.8 & 2006-05-01 & 21.7 & 1.5 & 1.1 & 7100 & 6.147 & 6330 & 8.8 & 10 \\
\hline 2453857.6 & 2006-05-02 & 22.5 & 1.5 & 1.1 & 7100 & 6.147 & 6330 & 8.8 & 10 \\
\hline 2453858.7 & 2006-05-03 & 23.6 & 2.0 & 1.4 & 6800 & 7.26 & 5810 & 10.1 & 10 \\
\hline 2453859.6 & 2006-05-04 & 24.5 & 2.0 & 1.4 & 6800 & 7.26 & 5810 & 10.1 & 10 \\
\hline 2453862.7 & 2006-05-07 & 27.6 & 2.0 & 1.7 & 6420 & 8.90 & 4550 & 17.9 & 10 \\
\hline 2453867.7 & 2006-05-12 & 32.6 & 2.0 & 1.7 & 6420 & 8.90 & 4550 & 17.9 & 10 \\
\hline 2453876.7 & 2006-05-21 & 41.6 & 2.0 & 1.8 & 6300 & 9.28 & 4320 & 30.3 & 10 \\
\hline 2453891.7 & 2006-06-05 & 56.6 & 2.0 & 1.8 & 6160 & 9.73 & 3760 & 50.7 & 10 \\
\hline 2453907.6 & 2006-06-21 & 72.5 & 2.0 & 1.9 & 6050 & 10.18 & 3160 & 79.4 & 10 \\
\hline
\end{tabular}

Note. - For each date in our sample of observations, we provide the following CMFGEN model parameters: Base comovingframe luminosity $L_{\mathrm{CMF}, R_{0}}$ and emergent observer-frame luminosity $L_{\mathrm{OBS}, R_{\mathrm{Max}}}$ (in $\mathrm{L}_{\odot}$ ), photospheric conditions describing the electron temperature $T_{\text {phot }}$ (in $\mathrm{K}$ ), the radius $R_{\text {phot }}$ (in $10^{14} \mathrm{~cm}$ ), the velocity $v_{\text {phot }}$ (in $\mathrm{km} \mathrm{s}^{-1}$ ), and the density (in $10^{-14} \mathrm{~g} \mathrm{~cm}{ }^{-3}$ ), together with the density exponent $n$ characterizing the density law $\rho(r)=\rho_{\text {phot }}\left(R_{\text {phot }} / r\right)^{n}$. (See 33 for a presentation of the modeling approach). ${ }^{a}: \Delta t$ is the time elapsed since discovery.

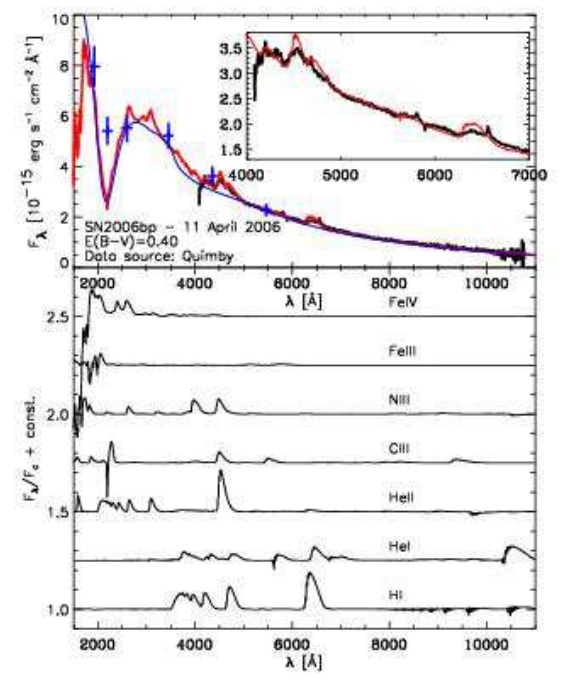

FIG. 8.- Top: Comparison between the reddened $(E(B-V)=$ 0.4 ) synthetic flux (red: full; blue: continuum only; $T_{\text {phot }}=$ $20,600 \mathrm{~K}$ ) and SN 2006bp observations on the 11th of April 2006, including the optical spectrum of Quimby (2007; black) and Swift UVOT fluxes (blue crosses). Note that, despite the larger extinction (responsible for the $2200 \AA$ dip), the UV flux level is comparable to that observed for SN 2005cs at discovery (shown in Fig. 3), suggesting a much higher ejecta temperature and ionization. Bottom: Rectified synthetic spectra for the model shown at top, but including bound-bound transitions of individual species (see label on the right). Apart from the standard $\mathrm{H}$ I and He I lines,

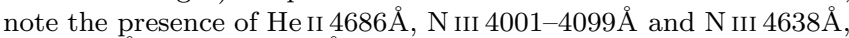

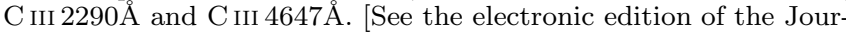
nal for a color version of this figure, and see 5.1 for discussion.]

in Table 7 and corresponding synthetic spectral fits to

torello et al. 2002), 1995N (Fox et al. 2000), 1997eg (Salamanca et al. 2002), 1998S (Pooley et al. 2002). Type IIn SNe are strong radio and X-ray emitters, associated with the interaction between the SN ejecta and the CSM, and with an X-ray luminosity that can rival the optical luminosity, as in 1995N (Fox et al. 2000).

\section{observations are presented in Figs.8 12}

\subsection{First week of evolution of SN 2006bp}

The first spectral observation of SN 2006bp, shown in Fig. 8, is impressive in several regards. It has a nearly featureless spectrum, with a very steep slope. The dip at $\sim 2200 \AA$ is not caused by line blanketing, as in the first spectrum of SN 2005cs (Fe IV is the main blocking ion in the UV, and it does so blueward of $1800 \AA$; its effect on the synthetic continuum SED is shown). The dip stems from interstellar extinction, with an inferred value $E(B-V)=0.4$. We also see, at all epochs, NaI D as a narrow absorption feature, indicating significant column density in the line of sight to SN 2006bp. Hydrogen Balmer lines are present, but very weak and very broad, and limited to $\mathrm{H} \alpha, \mathrm{H} \beta$, and $\mathrm{H} \gamma$. He I $5875 \AA$ is just barely identifiable. It is surprising that lines of N II and O II identified in the first spectra taken of SN 2005cs are not seen here. Given the high reddening, fitting the steep slope of the SED requires much higher temperatures and ionization conditions (Fig. 14). Consequently, CMFGEN predicts, although somewhat too strong, the presence of He II $4686 \AA$ (together with a small, $\sim 20 \%$ contribution from C III $4650 \AA$ ), a line that is more typical of the photosphere/wind of early-type $\mathrm{O}$ and WolfRayet stars (Hillier \& Miller 1999; Dessart et al. 2000; Crowther et al. 2002; Hillier et al. 2003), as well as

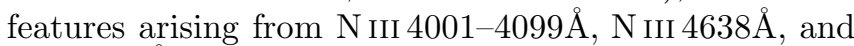
C III $2290 \AA$ (see bottom panel of Fig. 8). In practice, we used $T_{\text {phot }}=20,600 \mathrm{~K}, v_{\text {phot }}=14,100 \mathrm{~km} \mathrm{~s}^{-1}$, and $n=50$. Numerous directions in the parameter space were explored to reproduce the steepness of the SED, the small strength of P-Cygni profiles, and the strong blueshift of P-Cygni peak emission. These included varying the reddening and temperature, extent of the photosphere, ejecta chemistry, and density exponent. The most critical was the density exponent. Quimby et al. (2007) discuss the presence of weak and narrow lines 
in this optical spectrum, associated with HeII $4200 \AA$,

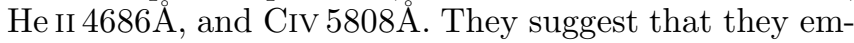
anate from material located just outside the SN ejecta that is flash ionized at shock breakout. This gives additional support to our identification here of HeII $4686 \AA$ emission from the SN ejecta itself. Note the photospheric velocity of SN 2005cs inferred from the first observed spectrum at 5 days after explosion is only $6880 \mathrm{~km} \mathrm{~s}^{-1}$, compared to $10430 \mathrm{~km} \mathrm{~s}^{-1}$ at a comparable epoch for SN $1999 \mathrm{em}$; see $\$ 4$ and DH06). We discuss further the properties of SN 2006bp at that age in $\$ 7.1$.

We could only reproduce this spectrum with CMFGEN by increasing the density exponent to 50 (see also 7.1 ). Because the density distribution is so steep, line and continuum formation coincide and occupy a very confined region of space at such early times. As time progresses, this region recedes, in the co-moving frame, to deeper layers where the density profile is flatter, but the spectrum formation region remains confined, which prevents the observer from simultaneously probing the outer regions. Changes in the exponent that describes the density distribution are best inferred through consecutive, rather than isolated, spectroscopic observations (Dessart \& Hillier 2005a). The peak emission of, e.g., the $\mathrm{H} \alpha$ line is blueward of its rest wavelength, corresponding to a velocity shift of $\sim 8000 \mathrm{~km} \mathrm{~s}^{-1}$ initially, but decreases with time to reach $\sim 1000 \mathrm{~km} \mathrm{~s}^{-1}$ on the 21 st of June. This is consistent with a large density exponent (Dessart \& Hillier 2005a), although our overestimate of the observed $\mathrm{H} \alpha$-peak blueshift on the 11th of April (Fig. 8) suggests that $n$ may not be as high as 50 , and that the weakness of optical lines may not be caused entirely by the steep density fall-off.

We show, in the top left panel of Fig. 9. CMFGEN fits to observations of SN 2006bp on the 12th of April 2006. The spectrum looks very similar to that on the 11th, the fit quality is superior, and the model differs in having a lower photospheric temperature and velocity. The model parameters are $T_{\text {phot }}=18,120 \mathrm{~K}$, $v_{\text {phot }}=12,550 \mathrm{~km} \mathrm{~s}^{-1}$, and $n=50$. The synthetic UV flux also closely matches the flux-equivalent $S$ wift UVOT magnitudes, with a nearly featureless spectrum strongly extinguished by the high reddening in the line of sight of $E(B-V)=0.4$. Along a low extinction line of sight, the flux at $\sim 2000 \AA$ would have been about ten times as large, reaching a level of $\sim 10^{-13} \mathrm{erg} \mathrm{cm}^{-2} \mathrm{~s}^{-1} \AA^{-1}$, i.e., about a hundred times greater than the optical flux at that time!

The HET observations on the 15th of April 2006 were of very low signal and were excluded from our sample. For the 17th of April 2006, we show CMFGEN fits in the top right panel of Fig. 9. The optical range shows hydrogen Balmer lines and He I $5875 \AA$, weak N II features in the blue edge of He I $5875 \AA$ and $\mathrm{H} \beta$, as well as a weak feature that we attribute to $\mathrm{O}$ II lines in the range 4300 $4600 \AA$ (see above discussion for SN 2005cs; Baron et al. 2007). The UV flux no longer dominates the optical and decreases rapidly from the combined effects of cooling (due to radiative losses and expansion) and increased line-blanketing. The optical lines now appear stronger and are well fitted with a density exponent of 20. The model parameters for the 17th of April 2006 are $T_{\text {phot }}=11,800 \mathrm{~K}, v_{\text {phot }}=11,300 \mathrm{~km} \mathrm{~s}^{-1}$, and $n=20$.
Two days later, on the 19th of April 2006, the spectrum looks very similar (bottom left panel of Fig. 9), with optical lines somewhat stronger and a diminishing UV flux. The model parameters are $T_{\text {phot }}=11,400 \mathrm{~K}$, $v_{\text {phot }}=11,050 \mathrm{~km} \mathrm{~s}^{-1}$, and $n=16$. Note the decreasing density exponent from 50 to 16 in just one week, and the decreasing photospheric velocity and temperature. Because the density distribution flattens, the outer ionized regions contribute more to the photospheric optical depth, shifting it outward to a lower density (Table 7). The same holds on the following day, the 20th of April 2006 (Fig. 9, bottom right panel), for which the model parameters are $T_{\text {phot }}=10,700 \mathrm{~K}, v_{\text {phot }}=10,300 \mathrm{~km} \mathrm{~s}^{-1}$, and $n=12$. The best-fit density exponent has a value of 12 , and changes little after this time to reach the canonical value of 10 . The spectral evolution is very slow and no new lines are identified in the spectrum. N II and O II lines have, however, disappeared, following the recombination of the ejecta.

On the 21st of April 2006, we have two sources of observations. The data of Quimby et al. (2007) starts only at $\sim 4200 \AA$ but extends out to $10700 \AA$, revealing a weak O I $7770 \AA$ and the absence of the CaII multiplet at $8500 \AA$ (left panel of Fig. 10). The Swift UVOT data points are well matched by the synthetic UV flux distribution, although we overestimate the U-band flux somewhat. The model parameters are $T_{\text {phot }}=10,700 \mathrm{~K}$, $v_{\text {phot }}=10,300 \mathrm{~km} \mathrm{~s}^{-1}$, and $n=12$. In the right panel of Fig. 10, we present a similar model with a photospheric temperature $\sim 1000 \mathrm{~K}$ lower, allowing a better match to the UV range, but a poorer match to the optical lines. This cooler model seems to have the right ionization for the UV flux, but has too low an ionization to explain the optical. We surmise that this stems from time-dependent effects, present at all times, but more visible in spectral lines of species (here, helium) that are about to change their ionization state in the ejecta (Dessart \& Hillier 2007a,b). This cooler model fits much better the weak Fe II $5169 \AA$ that indicates the start of the recombination of FeIII to FeII, together with the recombination of hydrogen. So this date closes the early epoch of SN 2006bp corresponding to fully ionized conditions in the photosphere region.

\subsection{The recombination epoch of $S N 2006 b p$}

From this epoch onwards, the ejecta in the photospheric layers is too cool to radiate a substantial UV flux, and line blanketing, due in particular to Fe II lines, blocks whatever UV light is produced, re-processing it to longer wavelengths. Extinction plays only a secondary role.

On the 24th of April 2006 (Fig. 11, top left panel), in addition to the strengthening hydrogen Balmer lines and the weakening He I $5875 \AA$, we see the appearance of spectral features due to Fe II lines, around $4400 \AA$ and $5200 \AA$ (the main contributor there is Fe II $5169 \AA$ ) and Mg II $4480 \AA$. The model parameters are $T_{\text {phot }}=9350 \mathrm{~K}$, $v_{\text {phot }}=7920 \mathrm{~km} \mathrm{~s}^{-1}$, and $n=10$. Note that we set the density exponent to 10 for this and all following dates.

On the 25th of April 2006 (Fig. 11, top right panel), the lines of Fe II strengthen and we see the appearance of Si II $6355 \AA$, although not as clearly as for SN 2005cs due to the higher photospheric velocities. It is not clear if the observed dip at around $5800 \AA$ is due to weakening 

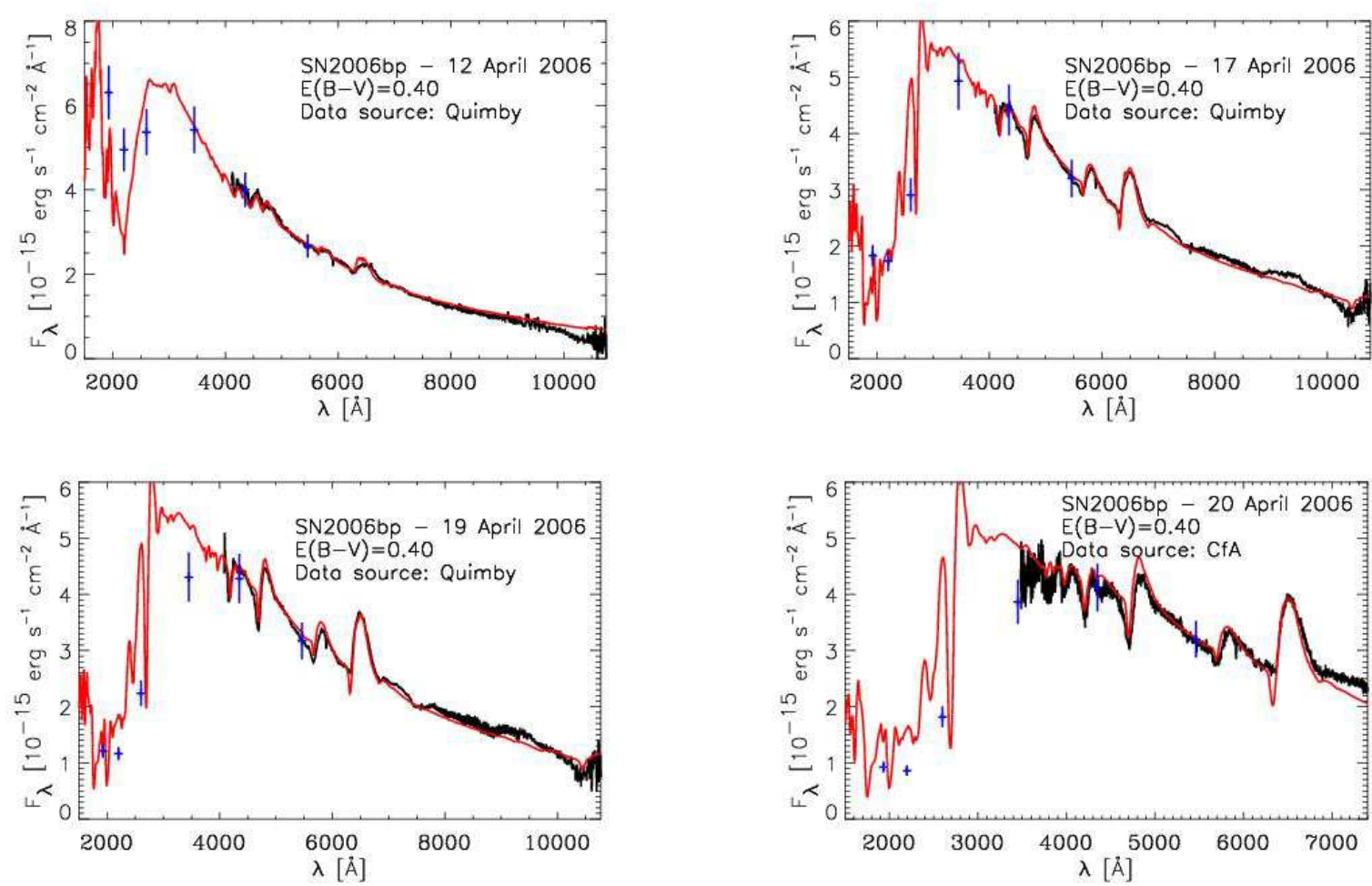

FIG. 9. - Same as Fig. 8 but for the SN 2006bp observations of April 12 th (top left; $T_{\text {phot }}=18120 \mathrm{~K}$ ), 17 th (top right; $T_{\text {phot }}=11800 \mathrm{~K}$ ), 19 th (bottom left; $T_{\mathrm{phot}}=11400 \mathrm{~K}$ ), and 20th (bottom right; $T_{\mathrm{phot}}=10700 \mathrm{~K}$ ), 2006. A full set of model parameters is given in Table 7 [See the electronic edition of the Journal for a color version of this figure, and see 5.1 for discussion.]
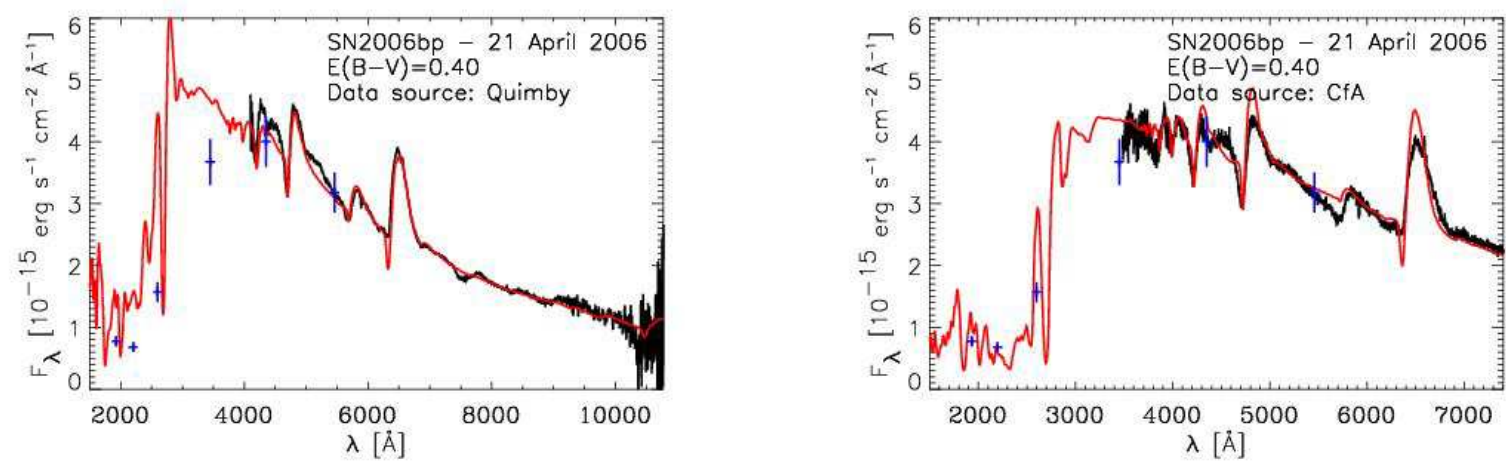

FIG. 10.- Same as Fig. 8 but for the SN 2006bp observations on the 21st of April 2006, using the data of Quimby et al. (2007; left) or the CfA data (right). The models used in each panel differ mostly in their temperature/ionization, with $T_{\text {phot }}=10700 \mathrm{~K}$ (left; Table 7 ) and $T_{\text {phot }}=9700 \mathrm{~K}$ (right). [See the electronic edition of the Journal for a color version of this figure, and see 5.1 for discussion.]

He I $5875 \AA$ (as supported by time-dependent calculations which predict a higher ejecta ionization sustained over a longer time) or due to the strengthening $\mathrm{NaID}$. In the red part of the spectrum, we also see the unambiguous presence of OI $7770 \AA$ and the weak appearance of the Ca II $8500 \AA$ multiplets, which are not predicted by CMFGEN at this epoch.

$\mathrm{H} \alpha$ is somewhat overestimated both in emission and absorption, which may be related to the assumption of steady state or to the constant density exponent adopted at a given epoch. The model parameters are $T_{\text {phot }}=$ $8150 \mathrm{~K}, v_{\text {phot }}=8750 \mathrm{~km} \mathrm{~s}^{-1}$, and $n=10$.

The spectrum of SN 2006bp on the 30th of April 2006 (middle left panel of Fig. 11) shows evidence of recombination in the ejecta. In particular, Fe II lines are now clearly visible throughout the $V$-band, affecting the $\mathrm{H} \alpha$ trough (with a small contribution from Si II $6355 \AA$ ). CaII lines at $3800 \AA$ and $8500 \AA$ are also strong and broad. NaID is the likely contributor to the dip at $5800 \AA$. At this epoch, we employ enhanced oxygen and carbon abundance compared to the starting conditions $(\mathrm{C} / \mathrm{He}=0.0017$ and $\mathrm{O} / \mathrm{He}=0.01$ by number $)$, while nitrogen is depleted $(\mathrm{N} / \mathrm{He}=0.0068)$ and nitrogen lines are not seen. In the red part of the spectrum, we observe numerous $\mathrm{C}$ I lines due to multiplets around $9100 \AA, 9400 \AA$, and $9630 \AA$. We also predict additional contributions from O I at $7990 \AA, 8450 \AA$, and $9260 \AA$, but all much weaker than the strong O I $7770 \AA$. The model parameters are $T_{\text {phot }}=7320 \mathrm{~K}, v_{\text {phot }}=6450 \mathrm{~km} \mathrm{~s}^{-1}$, and $n=10$.

For the CfA observations of the 1st and 2nd of May 

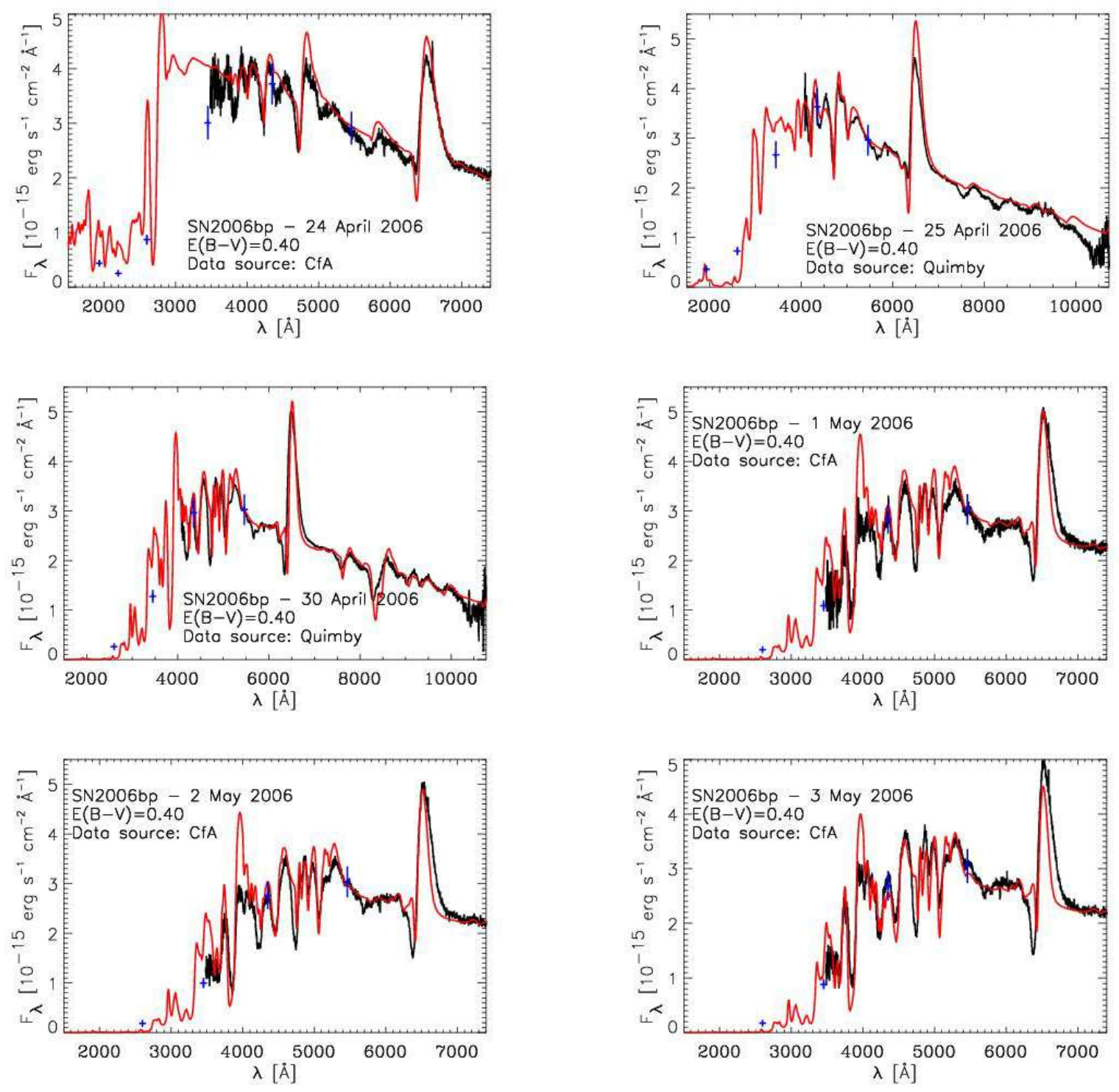

FIG. 11. - Same as Fig. 8 but for the SN 2006bp observations of April 24th (top left; $T_{\text {phot }}=9350 \mathrm{~K}$ ), 25 th (top right; $T_{\text {phot }}=8150 \mathrm{~K}$ ), 30 th $\left(\right.$ middle left; $\left.T_{\text {phot }}=7320 \mathrm{~K}\right)$, and May 1st $\left(\right.$ middle right; $\left.T_{\text {phot }}=7100 \mathrm{~K}\right)$, 2nd (bottom left; $\left.T_{\text {phot }}=7100 \mathrm{~K}\right)$, and $3 \mathrm{rd}($ bottom right; $T_{\text {phot }}=6800 \mathrm{~K}$ ), 2006. A full set of model parameters is given in Table 7 [See the electronic edition of the Journal for a color version of this figure, and see $\$ 5.2$ for discussion.]

2006bp (middle right and bottom left panels in Fig. 11), we employ the same model with $T_{\text {phot }}=7100 \mathrm{~K}, v_{\text {phot }}=$ $6330 \mathrm{~km} \mathrm{~s}^{-1}$, and $n=10$. The spectral range misses the red part of the spectrum where the oxygen, carbon, and calcium lines of interest would be found. The spectral evolution is slow: we see a slight fading of the flux in the blue, with a modest recession of the photosphere, as shown by the narrower line profiles.

We now discuss the late-time observations of SN 2006bp, an epoch that corresponds, in our steady-state modeling with CMFGEN, to conditions where hydrogen has recombined at and above the photosphere, and where the density of free electrons is low.

\subsection{Late evolution in the photospheric phase of $S N$ $2006 b p$}

We present in Fig. 12 a montage of synthetic spectra fitted to observations of SN 2006bp on May 4th (top left), 7th (top right), 12th (middle left), 21st (middle right), and June 5th (bottom left) and 21st (bottom right), 2006. In this elapsed time, the model photospheric temperature (velocity) decreases steadily from $6800 \mathrm{~K}\left(5810 \mathrm{~km} \mathrm{~s}^{-1}\right)$ to $6050 \mathrm{~K}\left(3160 \mathrm{~km} \mathrm{~s}^{-1}\right)$, while the luminosity and the density exponent remain constant (a full set of model parameters is given in Table 7).

Throughout this period, the UV flux fades and the SED peaks in the optical. The continuum energy distribution is very well fitted at all times, becoming more and more affected by metal line blanketing, whose effect appears first in the optical with Fe II lines. Contributions from $\mathrm{Ti}$ II lines in the $4200 \AA$ region strengthen with time and cause the $B$-band magnitude to plummet. By the 21st of June, Fe II lines even affect the flux in the red part of the spectrum, around $7300 \AA$, while $\mathrm{O}$ I and $\mathrm{C}$ I lines continue to contribute numerous narrow features in the red. Ca II H\&K is overestimated early on, while the Ca II multiplet at $8500 \AA$ is generally well fitted. We note the striking evolution of the $\mathrm{H} \alpha$ strength, 

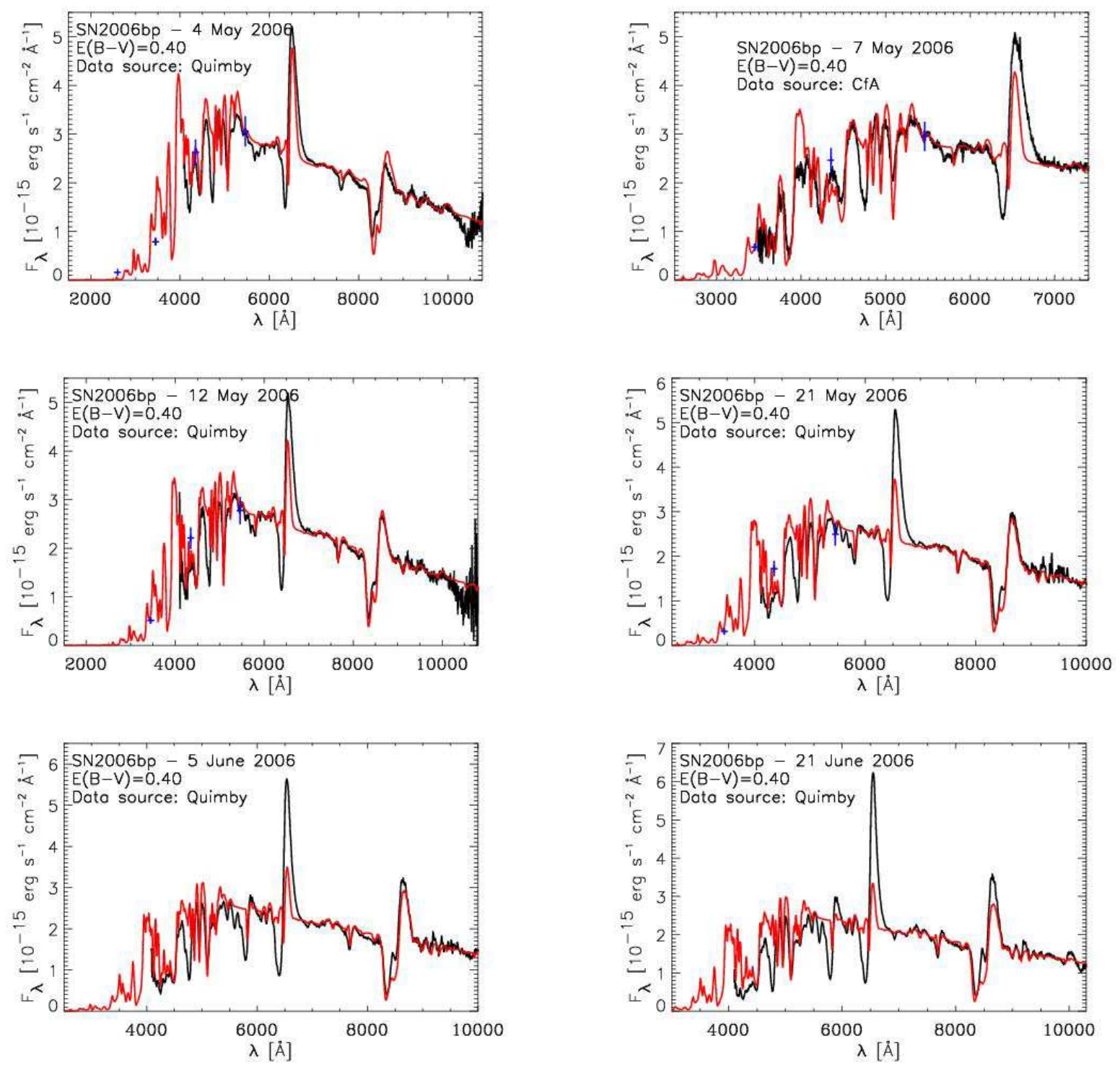

FIg. 12. - Same as Fig. 8 but for the SN 2006bp observations of May 4th (top left; $T_{\text {phot }}=6800 \mathrm{~K}$ ), 7 th (top right; $T_{\text {phot }}=6420 \mathrm{~K}$ ), 12 th $\left(\right.$ middle left; $\left.T_{\text {phot }}=6420 \mathrm{~K}\right), 21 \mathrm{st}\left(\right.$ middle right; $\left.T_{\text {phot }}=6300 \mathrm{~K}\right)$, and June 5 th (bottom left; $\left.T_{\text {phot }}=6160 \mathrm{~K}\right)$, and $21 \mathrm{st}(\mathrm{bottom}$ right; $T_{\text {phot }}=6050 \mathrm{~K}$ ), 2006. A full set of model parameters is given in Table 7 [See the electronic edition of the Journal for a color version of this figure, and see $\$ 5.2$ for discussion.]

observed with a fixed strength over this 6-week period, while steady-state CMFGEN models predict an ever decreasing strength and width (always underestimated) for that line (Fig. 12). This is caused by time-dependent effects, as proposed by Utrobin \& Chugai (2005) and Dessart \& Hillier (2007a,b), and can be remedied by accounting for the time-dependent terms in the statistical and radiative equilibrium equations. Other lines tend to be too narrow, in particular Na I D. The good fit to the central part of the line profile indicates that the photospheric velocity is well matched, but the extent of the corresponding emission line regions is systematically underestimated. So, while we provide fits to observations at those late epochs, time dependent models would yield different parameters and synthetic spectra more compatible with the observations. Since time dependence does not profoundly alter the continuum energy distribution, our synthetic fits may still be useful for distance determinations. It remains to be checked to what extent the effects introduced by time dependence affect the correction factors used in the EPM, the true magnitude of the flux dilution, etc (Dessart \& Hillier 2005b). In practice, we exclude the observations of SN 2006bp on the 5th and on the 21st of June for the determination of its distance.

\section{DISTANCES TO TYPE II-P SNe}

In this section, we compute distances and explosion times of SNe 2005cs and 2006bp using our photometric observations and synthetic spectra ${ }^{11}$. We use two methods, which emerged as the most suitable from the detailed discussion in Dessart \& Hillier (2005b) and DH06. The first approach is to apply the Expanding Photosphere Method (EPM; Kirshner \& Kwan 1974; Eastman \& Kirshner 1989; Schmidt et al. 1994; Eastman

11 Here, we associate the time of explosion with that of shock breakout, which may be about a day after core bounce, but corresponds well to the time when the entire envelope is turned into SN ejecta. 
et al. 1996; Hamuy et al. 2001; Leonard et al. 2002a; Elmhamdi et al. 2003), but using correction factors and color temperatures calculated directly from our tailored models at each epoch (as in DH06). This approach is superior to using analytical formulae describing the dependence of correction factors on color temperature, since those are computed for models that may not match the observed spectrum. This approach avoids the physical scatter that stems from such a diversity among SN II. The second approach is to use our synthetic spectra to directly fit observations, as described in DH06, in a similar fashion to the SEAM (Baron et al. 2004). For both, the observed magnitudes shown in Table 1 and 2 are used, either directly or interpolated linearly between adjacent dates to correspond to the date of the spectroscopic observation. This does not introduce any sizable error given the very slow change (nearly constant from day to day within the errors) of $B, V$, and $I$ magnitudes in the early photospheric phase of Type II-P SNe.

We have described at length the EPM in Dessart \& Hillier (2005b), complementing the previous work of Eastman et al. (1996), providing some insights into the method and its limitations, as well as a new and independently determined set of correction factors based on a large database of CMFGEN models. Correction factors determined with CMFGEN are comparable to, albeit systematically 10-20\% larger than, those of Eastman et al., although the origin of this small, but important, difference remains unclear. In DH06, we applied the EPM to SN 1999em and found that, unless the correction factors and associated color temperatures are determined with tailored models, the EPM distance and the explosion time were inconsistent when different bandpass-sets were used (amongst $B V, B V I$, and $V I$ ). Since the photospheric radius is nearly constant across the optical range (Dessart \& Hillier 2005b) the inferred distance ought to be the same whatever optical set is used.

Another source of uncertainty, although smaller, is the inference of the photospheric velocity, $v_{\text {phot, }}$ usually approximated by adopting the Doppler velocity at maximum absorption in weak and seemingly isolated $\mathrm{P}$ Cygni profiles (note that sphericity and projection effects, subtleties in the line transfer problem, and diversity in atomic properties lead to a non-trivial relation between the photospheric velocity and the velocity at maximum absorption in a given line; see Dessart \& Hillier 2005b for a discussion on this issue). In our approach, we adopt the value from our CMFGEN model. Although our model fits are satisfactory, they sometimes overestimate or underestimate profile widths, matching some profiles very well while doing a poorer job on others at the same time. There is likely a genuine range of velocities at the photosphere due to fluid instabilities and density variations, so that a $10 \%$ inaccuracy in the photospheric velocity at a given time may in fact exist. However, we expect the sense and the magnitude of the uncertainty in our inferences to behave randomly. We do not consistently overestimate or underestimate $\mathrm{v}_{\text {phot }}$, or the flux in a given spectral region, so that observations at many times should reduce the uncertainty.

Reddening is another ingredient requiring special care in distance estimates. In our approach, we use synthetic SEDs to constrain the reddening $E(B-V)$ within \pm 0.05 . The dust properties in other galaxies may not be well re- produced by the Cardelli law, which we employ, and this introduces an additional uncertainty, which is significant for the strongly extinguished SN 2006bp (for such a discussion, see, e.g., Elias-Rosa et al. 2006).

For both objects, we have a good constraint on the explosion time from prior optical detection/non-detection. However, compact progenitor stars like blue supergiants brighten in the optical much more quickly than RSG stars with their extended envelopes, whose SED may peak in the UV a few days after explosion (the difference comes from the energy penalty associated with the ejecta expansion). Hence, optical non-detection/detection may not constrain the explosion date to better than one day. Searching for the UV and X-ray signatures at shock breakout would be more valuable in this respect.

\section{1. $S N 2005 c s$}

The high quality spectral fits to the 13 photosphericphase observations of SN 2005cs presented in the preceding sections are well suited to determine the distance to the SN and its host galaxy M 51a (NGC 5194).

Focusing first on the EPM, we calculated the correction factors and color temperatures for all dates in our SN 2005cs sample. As in DH06, we employ three bandpass sets, using $\{\mathrm{B}, \mathrm{V}\},\{\mathrm{B}, \mathrm{V}, \mathrm{I}\}$, and $\{\mathrm{V}, \mathrm{I}\}$. We $\log$ in Table 8 the color temperature and associated correction factors for each model. Both quantities follow the same trend discussed in Dessart \& Hillier (2005b) or Eastman et al. (1996), with comparable values to those found at comparable epochs for SN 1999em (DH06). At late times corresponding to the recombination epoch, the correction factors rise for $\{\mathrm{B}, \mathrm{V}\}$ and $\{\mathrm{B}, \mathrm{V}, \mathrm{I}\}$, driven by the strong Fe II and Ti II line blanketing in the $B$-band. The resulting fading of the $B$-band magnitude is, thus, not related to a variation in the flux-dilution magnitude. Also given in Table 8 are the angular sizes resulting from the EPM, at each date. Note that these are determined with an estimated accuracy of $\lesssim 10 \%$, which is a minimum error that accounts for the mismatch between synthetic and observed spectra. The SN 2005cs photosphere approximately doubles in radius within one month of explosion, a comparable increase to that inferred for SN 1999em. Using the model photospheric velocity (Table 6) together with these angular sizes, we use the method of least squares to fit the equation $\theta / v_{\text {phot }}=\left(t-t_{\text {explosion }}\right) / D$, taking time as the independent variable, and list the resulting distance and explosion dates in Table 8

Using either the first 12 dates (to test the effect of a shorter time baseline of two weeks, we exclude the last date, corresponding to the recombination epoch) or all 13 dates (encompassing four weeks), we find a distance of $8.9 \pm 0.5 \mathrm{Mpc}$ and an explosion that occurred on JD $2453547.6 \pm 0.5$, i.e., 2.8 days prior to discovery ( $\sim 5$ days prior to the first observation). The agreement between the three bandpasses is good. Lengthening the time baseline by two weeks by adding the thirteenth date yields the same results, which provides further credence to the reliability of this result. A plot of these EPM data points for the $B V I$ passband, together with a linear fit to the data, are shown in Fig. 13 (top curve).

The second approach is to fit directly the observations with our models. Assuming homologous expansion and adopting the model $v_{\text {phot }}$ (as in the EPM), we select an explosion time to obtain a photospheric radius at each 
ferred explosion date is compatible within errors with the constraint of $2453549 \pm 1$ imposed by prior non-detection (P06), since even after the explosion has occurred, optical brightening is not instant. Adopting a $10 \%$ uncertainty in the inferred angular size (which corresponds to a minimum error associated with fitting mismatches) leads to uncertainties of $0.5 \mathrm{Mpc}$ and 0.5 day for the distance and explosion date determined through the EPM. The additional uncertainties in photospheric velocity, reddening, and photometry, are small, so that, through our use of many observations, our inferred distance should be accurate to within $\lesssim 10 \%$.

Our inferred distance is slightly larger than two recent EPM-like distance measurements to SN 2005cs. Using PHOENIX version 14 (Hauschildt \& Baron 1999), Baron et al. (2007) obtained a SEAM distance to SN $2005 \mathrm{cs}$ of $7.9_{-0.6}^{+0.7} \mathrm{Mpc}$ using all $U B V R I$ magnitudes, but at only two epochs. In some sense the level of agreement is satisfying: PHOENIX and CMFGEN are two very distinct codes, and use different assumptions in the modeling. Takáts \& Vinkó (2006) used the EPM (with analytical correction factors and an estimate of the reddening $E(B-V)=0.1)$ and the standard candle method of Hamuy \& Pinto (2002) to determine a distance of $7.59 \pm 1.02 \mathrm{Mpc}$ and $6.36 \pm 1.3 \mathrm{Mpc}$, respectively. Their EPM distance was actually an average of two determinations - one in which the explosion time was fixed $(d=8.34 \pm 0.31)$ and one in which the explosion time was a free parameter $(\mathrm{d}=6.84 \pm 0.18)$. The later gave a better fit to the data, but yielded an explosion time 4 days later than that inferred directly from the observations. Takáts \& Vinkó (2006) find a challenging enhancement in the photospheric angular size of about 10-20 over the same period, which, in light of our CMFGEN model results, would suggest an outward migration, in the Lagrangean (or co-moving) frame, of the photosphere with time. At later times our results, and those of Takáts \& Vinkó, appear to be consistent; at early times Takáts \& Vinkó estimate much hotter photospheric temperatures, and smaller photospheric radii.

In general, our inferred distance is larger than most other estimates in the literature. Previously, using lightcurve or spectroscopic modeling of the Type Ic SN 1994I, Iwamoto et al. (1994) and Baron et al. (1996) obtained a distance of $6.9 \pm 1 \mathrm{Mpc}$ and $6 \pm 1.9 \mathrm{Mpc}$, respectively, although the reddening may be uncertain in the line of sight to this object. Georgiev et al. (1990) obtained a distance of $6.9 \pm 0.7 \mathrm{Mpc}$ using surface photometry of stellar associations. Feldmeier et al. (1997) found a distance of $8.4 \pm 0.6 \mathrm{Mpc}$ using planetary nebulae, revised downward by Ciardullo et al. (2002) to $7.6 \pm 0.6 \mathrm{Mpc}$ with an improved reddening of 0.04. Note that this is the reddening we infer in the line of sight to SN 2005cs. Tonry et al (2001) infer a distance to NGC 5195 (M 51b, a companion galaxy to $\mathrm{M} 51$ ) of $7.66 \pm 1 \mathrm{Mpc}$ from surface brightness fluctuations. Our estimate is compatible, within the errors, with that of Tonry et al. (2001), but mildly in disagreement with the other studies. Obtaining a Cepheid distance to M 51 would help in pinning down the distance to that Galaxy and in providing an independent test for the accuracy of our distance estimate.

6.2. $S N$ 2006bp
We now apply the methods of the previous section to SN 2006bp. To avoid the hydrogen-recombination phase, when steady-state CMFGEN models struggle to reproduce the hydrogen Balmer and Na I D lines, we exclude the two extreme observations in our sample, those of the 5 th and of the 21st of June 2006, leaving us with 16 observations and a time coverage of 4 weeks.

We present the EPM-inferred quantities in Table 10. giving the angular size, correction factors and color temperatures for the three sets of bandpasses $B V, B V i^{\prime}$, and $V i^{\prime}$. Because of the faster expansion of SN 2006bp compared to SN 2005cs, the photospheric angular size more than triples over the first month of observation. At the recombination epoch, the associated front, which coincides closely with the photosphere, recedes in the co-moving frame at a rate that is comparable to the expansion rate of the ejecta. At late times, the angular size of the photosphere is, thus, roughly constant.

Using all 16 observations in our sample and our EPMbased technique leads to a distance of $17.5 \pm 0.6 \mathrm{Mpc}$ for the $B V i^{\prime}$ set, only slightly varying with bandpass set $(17.9 \pm 0.7 \mathrm{Mpc}$ for the $B V$ set and $17.4 \pm 0.6 \mathrm{Mpc}$ for the $V i^{\prime}$ set; Table 10). Including only the first 13 dates (to assess the effect of a shorter time baseline of one month) leads to a higher distance by $0.2-0.3 \mathrm{Mpc}$ in each band and a larger error of $\pm 0.8 \mathrm{Mpc}$. The explosion date is also in good agreement for either 13 or 16 dates in our sample, with a Julian date of $2453833.4 \pm 0.4$ day. A plot of these EPM data points for the $B V i^{\prime}$ passband, together with a linear fit to the data, are shown in Fig. 13 (bottom curve).

We also use our synthetic spectra and magnitudes to constrain directly the distance, in a fashion similar to the SEAM. We find a distance in agreement with the EPM inference, with $17.1 \mathrm{Mpc}$ for the $B V i^{\prime}$ set (Table 11). Uncertainties are larger than the scatter of values across bandpasses, but should remain at the 5-10\% level, in particular due to the large number of observations. Errors are unlikely to be purely statistical, but they have some level of randomness as the deviations from a perfect match of synthetic to observed spectra are very different for individual epochs (blue featureless spectrum at early time to strongly blanketed spectrum at the recombination epoch), different spectral regions (the blue and red wavelength regions reflect different contributions from different elements that we model with variable levels of success). Reducing the number of observations to 9 dates increases the distance estimate by $1.7 \mathrm{Mpc}$. Uncertainties in reddening and photospheric velocity have a similar effect to that described for SN 2005cs (\$4) and SN 1999em (DH06).

To conclude, we estimate a distance to SN 2006bp and its galaxy host NGC 3953 of $17.5 \pm 0.8 \mathrm{Mpc}$ together with an explosion date of $2453833.4 \pm 0.4$ day only 1.7 days after discovery ( $\sim 3$ days prior to the first observation). This makes it the earliest-discovered Type II-P SN and gives further support to the novel properties of the SN ejecta inferred from the first observed spectra (steep density distribution, highly ionized ejecta with He II $4686 \AA$ line emission). This distance estimate is in close match to the value of $17 \mathrm{Mpc}$ obtained by Tully (1988). This explosion date is also in agreement with the date of April 9th, inferred by Quimby et al. (2007) from fits to optical 
TABLE 10

EPM QUANTITIES FOR, AND EPM-BASED DISTANCE TO, SN2006BP

\begin{tabular}{|c|c|c|c|c|c|c|c|c|c|c|}
\hline \multirow[t]{2}{*}{ Julian Date } & \multirow[t]{2}{*}{ Day } & \multicolumn{3}{|c|}{$\begin{array}{c}\text { Angular Size } \\
\left(10^{8} \mathrm{~km} \mathrm{Mpc}^{-1}\right)\end{array}$} & \multicolumn{3}{|c|}{ Correction Factor } & \multicolumn{3}{|c|}{$\begin{array}{c}\text { Color Temperature } \\
(\mathrm{K})\end{array}$} \\
\hline & & $\theta_{B V}$ & $\theta_{B V i^{\prime}}$ & $\theta_{V i^{\prime}}$ & $\xi_{B V}$ & $\xi_{B V i^{\prime}}$ & $\xi_{V i^{\prime}}$ & $T_{B V}$ & $T_{B V i^{\prime}}$ & $T_{V i^{\prime}}$ \\
\hline 2453836.6 & 2006-04-11 & 2.345 & 2.373 & 2.352 & 0.586 & 0.663 & 0.788 & 24899 & 21736 & 17632 \\
\hline 2453837.6 & 2006-04-12 & 2.856 & 2.884 & 2.842 & 0.563 & 0.657 & 0.734 & 21916 & 18753 & 16511 \\
\hline 2453842.6 & $2006-04-17$ & 4.838 & 4.929 & 4.873 & 0.499 & 0.557 & 0.608 & 15330 & 14009 & 12847 \\
\hline 2453844.6 & 2006-04-19 & 5.111 & 5.216 & 5.160 & 0.480 & 0.538 & 0.589 & 15030 & 13708 & 12547 \\
\hline 2453845.8 & 2006-04-20 & 5.580 & 5.685 & 5.615 & 0.454 & 0.512 & 0.560 & 14489 & 13188 & 12107 \\
\hline 2453846.7 & 2006-04-21 & 5.524 & 5.643 & 5.580 & 0.454 & 0.512 & 0.560 & 14489 & 13188 & 12107 \\
\hline 2453849.6 & $2006-04-24$ & 6.239 & 6.351 & 6.365 & 0.422 & 0.490 & 0.544 & 13548 & 12127 & 11026 \\
\hline 2453850.6 & 2006-04-25 & 7.527 & 7.569 & 7.597 & 0.451 & 0.496 & 0.531 & 11126 & 10445 & 9864 \\
\hline 2453855.7 & 2006-04-30 & 7.772 & 7.884 & 7.940 & 0.583 & 0.525 & 0.486 & 8963 & 9524 & 10125 \\
\hline 2453856.8 & 2006-05-01 & 7.891 & 8.003 & 8.039 & 0.634 & 0.544 & 0.486 & 8423 & 9164 & 10025 \\
\hline 2453857.6 & 2006-05-02 & 7.828 & 7.961 & 8.032 & 0.634 & 0.544 & 0.486 & 8423 & 9164 & 10025 \\
\hline 2453858.7 & 2006-05-03 & 8.165 & 8.249 & 8.333 & 0.692 & 0.576 & 0.502 & 7802 & 8583 & 9524 \\
\hline 2453859.6 & 2006-05-04 & 8.039 & 8.165 & 8.263 & 0.692 & 0.576 & 0.502 & 7802 & 8583 & 9524 \\
\hline 2453862.7 & 2006-05-07 & 8.627 & 8.732 & 8.823 & 0.862 & 0.653 & 0.531 & 6681 & 7562 & 8743 \\
\hline 2453867.7 & 2006-05-12 & 8.172 & 8.396 & 8.571 & 0.862 & 0.653 & 0.531 & 6681 & 7562 & 8743 \\
\hline \multirow[t]{2}{*}{2453876.7} & 2006-05-21 & 7.969 & 8.200 & 8.375 & 1.058 & 0.724 & 0.560 & 6001 & 7022 & 8343 \\
\hline & & \multicolumn{3}{|c|}{$B V$ set } & \multicolumn{3}{|c|}{$B V i^{\prime}$ set } & \multicolumn{3}{|c|}{$V i^{\prime}$ set } \\
\hline \multicolumn{11}{|c|}{ Using First 13 Dates } \\
\hline \multirow{2}{*}{\multicolumn{2}{|c|}{$\begin{array}{c}\mathrm{D}(\mathrm{Mpc}) \\
\mathrm{t}_{\text {explosion }}(\mathrm{JD})\end{array}$}} & \multirow{2}{*}{\multicolumn{3}{|c|}{$\begin{array}{c}18.1 \pm 0.8 \mathrm{Mpc} \\
2453833.2 \pm 0.4\end{array}$}} & \multirow{2}{*}{\multicolumn{3}{|c|}{$\begin{array}{c}17.8 \pm 0.8 \mathrm{Mpc} \\
2453833.3 \pm 0.4\end{array}$}} & \multirow{2}{*}{\multicolumn{3}{|c|}{$\begin{array}{c}17.7 \pm 0.8 \mathrm{Mpc} \\
2453833.3 \pm 0.4\end{array}$}} \\
\hline & & & & & & & & & & \\
\hline \multicolumn{11}{|c|}{ Using All 16 Dates } \\
\hline \multicolumn{2}{|c|}{$\begin{array}{l}\mathrm{D}(\mathrm{Mpc}) \\
\text { splosion (JD) }\end{array}$} & \multicolumn{3}{|c|}{$\begin{array}{l}17.9 \pm 0.7 \mathrm{Mpc} \\
2453833.3 \pm 0.3\end{array}$} & \multicolumn{3}{|c|}{$\begin{array}{c}17.5 \pm 0.6 \mathrm{Mpc} \\
2453833.4 \pm 0.3\end{array}$} & \multicolumn{3}{|c|}{$\begin{array}{c}17.4 \pm 0.6 \mathrm{Mpc} \\
2453833.4 \pm 0.3\end{array}$} \\
\hline
\end{tabular}

Note. - Summary of EPM quantities for SN 2006bp. The quantity $\theta / v$ is computed assuming a reddening $E(B-V)=0.4$. (See text for discussion.)

TABLE 11

DistANCE TO SN2006BP USING A DIRECT SPECTRAL-FITTING APPROACH.

\begin{tabular}{cccccc}
\hline \hline $\begin{array}{c}\text { texplosion } \\
(\mathrm{JD})\end{array}$ & $\begin{array}{c}\mathrm{d} \\
(\mathrm{Mpc})\end{array}$ & $E(B-V)$ & $\begin{array}{c}v_{\text {scale }} \\
\left(v_{\text {phot }}\right)\end{array}$ & Set & $n_{\text {obs }}$ \\
\hline 2453833.4 & 17.4 & 0.4 & 1.0 & $B V$ & 16 \\
2453833.4 & 17.1 & 0.4 & 1.0 & $B V i^{\prime}$ & 16 \\
2453833.5 & 16.7 & 0.4 & 1.0 & $V i^{\prime}$ & 16 \\
\hline 2453833.5 & 16.8 & 0.4 & 1.0 & $B V i^{\prime}$ & 15 \\
2453833.5 & 16.8 & 0.4 & 1.0 & $B V i^{\prime}$ & 14 \\
2453833.3 & 17.4 & 0.4 & 1.0 & $B V i^{\prime}$ & 13 \\
2453833.3 & 17.6 & 0.4 & 1.0 & $B V i^{\prime}$ & 12 \\
2453833.1 & 18.0 & 0.4 & 1.0 & $B V i^{\prime}$ & 11 \\
2453833.1 & 18.3 & 0.4 & 1.0 & $B V i^{\prime}$ & 10 \\
2453832.9 & 18.9 & 0.4 & 1.0 & $B V i^{\prime}$ & 9 \\
\hline 2453833.4 & 17.1 & 0.4 & 1.0 & $B V i^{\prime}$ & 16 \\
2453833.4 & 18.8 & 0.4 & 1.1 & $B V i^{\prime}$ & 16 \\
2453833.4 & 15.4 & 0.4 & 0.9 & $B V i^{\prime}$ & 16 \\
\hline 2453833.4 & 15.9 & 0.45 & 1.0 & $B V i^{\prime}$ & 16 \\
2453833.4 & 17.1 & 0.40 & 1.0 & $B V i^{\prime}$ & 16 \\
2453833.4 & 18.3 & 0.35 & 1.0 & $B V i^{\prime}$ & 16 \\
\hline $2453833.0^{a}$ & 17.6 & 0.4 & 1.0 & $B V i^{\prime}$ & 16 \\
$2453833.5^{a}$ & 16.9 & 0.4 & 1.0 & $B V i^{\prime}$ & 16 \\
$2453834.0^{a}$ & 16.2 & 0.4 & 1.0 & $B V i^{\prime}$ & 16 \\
& & & & & \\
\hline
\end{tabular}

NOTE. - Summary of SEAM results for SN 2006bp, as well as tests on dependencies with reddening, photospheric velocity, and number of observations used in the sample. In this approach, the error on the distance is of the order of $5-10 \%$, and corresponds to the minimum distance-modulus scatter between dates. ${ }^{a}$ : In this case, the distance is obtained by assuming the time of explosion, as shown. (See text for discussion.)

ROTSE-III light curve (note however that the SN may remain dim in the optical for many hours after breakout).

\section{DISCUSSION}

\subsection{Ejecta properties a few days after shock breakout}

We have presented quantitative spectroscopic analyses of two Type II-P SNe, using observations trig-

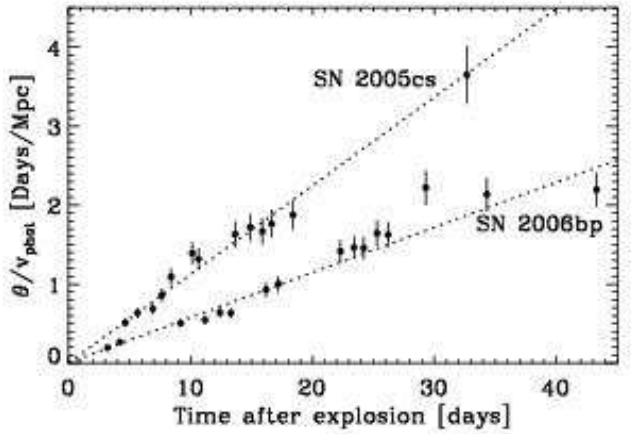

FIG. 13.- Linear fit (dotted line) to the $B V I$ data shown in Table 8 for SN $2005 \mathrm{cs}$ (using 13 dates) and to the $B V i^{\prime}$ data shown in Table 10 for SN2006bp (using 16 dates). The reciprocal of the slope is the distance $D$, i.e. $\theta / v_{\text {phot }}=\left(t-t_{\text {explosion }}\right) / D$, where $\theta=R_{\text {phot }} / D$. The origin for the time is the inferred time of explosion (more specifically the shock breakout time). The vertical bars indicate a $\pm 10 \%$ error.

gered at an exceptionally early time after explosion (3-5 days), providing a glimpse into the ejecta structure relatively soon after shock breakout. We infer a very high-temperature/very high ionization in the corresponding photospheric layers and in particular identify the presence of broad, ejecta-related, He II $4686 \AA$ in the first two observations of SN 2006bp (narrow emission in He II $4686 \AA$ is also identified by Quimby et al. (2007), related to material outside of the SN ejecta, which also testifies for the high temperature associated with shock breakout). In Fig. 14, we show the ionization level for $\mathrm{H}, \mathrm{He}, \mathrm{C}, \mathrm{N}, \mathrm{O}$, and $\mathrm{Fe}$ in the ejecta computed with CMFGEN and for models that fit the first spectroscopic and photometric observations of SN 2006bp (left panel), SN 2005cs (middle panel), and SN 1999em (right panel). These correspond, in the same sequence, to 3,5 , and 6 
days after explosion, as determined in this work and in DH06 ${ }^{12}$. One clearly sees in this figure the evolution to lower ionization through this sequence, following directly the elapsed time since explosion. Oxygen changes from being twice- to singly-ionized at mid-heights, from SN $2006 \mathrm{bp}$ to SN $1999 \mathrm{em}$. Helium is singly ionized throughout the SN 1999em ejecta, but twice ionized in the inner and outer regions of the SN 2006bp ejecta. This trend towards much higher ionization at earlier times supports the identification of He II $4686 \AA$ emission in the SN 2006bp ejecta, as well as O II lines for both SN 2006bp (at about 5 days after explosion) and SN 2005cs (in the discovery spectrum). O II lines were not detected unambiguously in the first spectrum taken of SN $1999 \mathrm{em}$ because it was taken too late, about 1-2 days later than SN 2005cs relative to the explosion time.

Moreover, at such early times, adopting the nitrogen abundance of DH06 now overestimates the strength of $\mathrm{N}$ II lines and underestimates the strength of $\mathrm{O}$ II lines, justifying the adoption in this work of $\mathrm{CNO}$ abundances closer to solar, i.e. $\mathrm{C} / \mathrm{He}=0.0004, \mathrm{~N} / \mathrm{He}=0.0013, \mathrm{O} / \mathrm{He}$ $=0.0016$, in agreement with the surface chemistry of blue supergiants analyzed by Crowther et al. (2006). By comparison, DH06 used $\mathrm{C} / \mathrm{He}=0.00017, \mathrm{~N} / \mathrm{He}=0.0068$, $\mathrm{O} / \mathrm{He}=0.0001$ (all given by number; for both works we use $\mathrm{H} / \mathrm{He}=5$ ). We thus concur with the findings of Baron et al. (2007) on the necessary CNO chemistry to reproduce the observations of $\mathrm{O}$ II and N II lines in the early spectra of SN 2005cs (as well as in SN 2006bp), with a depletion of carbon and oxygen compared to solar that is modest rather than severe. In general, an accurate determination of $\mathrm{CNO}$ abundances in the fast-expanding photospheres of Type II-P SNe is difficult since the corresponding lines are weak and overlap with lines of other species such as HeI. Having recourse to quantitative spectroscopy of the emergent light from their supergiant progenitors, which can be found in number and nearby, seems a more viable route to constraining the surface chemistry of such pre-SN massive stars.

In both objects, the near featureless spectral appearance over the first few days requires the choice of a very high power-law density exponent of 20-50 to dwarf the emission volume of all optical lines, as well as very high ejecta velocities to reproduce the large widths of emission line features. This is admittedly well above the value $n=10$ for the propagation of a shock wave through the envelope of a massive star progenitor, both obtained theoretically (Imshennik \& Nadezhin 1988; Shigeyama \& Nomoto 1990, Chevalier 1982; Ensman \& Burrows 1992, Blinnikov et al. 2000) and inferred observationally (see, e.g., Eastman \& Kirshner 1989; Schmutz et al. 1990; Baron et al. 2000; Dessart \& Hillier 2005a,2006a). Nonetheless, for the outermost layers of the progenitor envelope, a much steeper density profile is expected, with values in the vicinity of a few tens (Blinnikov et al. 2000, and in particular their Fig. 1), and, under certain conditions, a small amount of mass at the progenitor surface may even reach relativistic speeds as the shock breaks out (Matzner \& McKee 1999).

\footnotetext{
12 The maximum radius differs between simulations, resulting from the different density exponents, but in all cases is such that the radiation is freely-streaming at the outer grid radius (opticallythin conditions)
}

Unfortunately, the radiation-hydrodynamics codes used to compute the ejecta structures of core-collapse SN are Lagrangean, and are thus not well designed to describe the dynamics and the structure of the low density surface layers. In those fully-ionized regions, the luminosity is super-Eddington by up to a few orders of magnitude, even when accounting only for the electronscattering opacity. When accounting for the additional contribution of optically thick and thin lines, we find that the radiation pressure can be very substantial, following the combination of a huge luminosity, a low density, and a large velocity gradient (Castor, Abbott, \& Klein 1975). In the CMFGEN model of SN 2006bp on 30 June 2006, the radiative acceleration in the layers above the photosphere reaches a few thousand $\mathrm{cms}^{-2}$, which combined with a velocity of a few $10000 \mathrm{~km} \mathrm{~s}^{-1}$ and a length scale of $10^{14} \mathrm{~cm}$ lead to a velocity gain of few tens of percent (assuming the acceleration remains constant). Sooner after shock breakout, the radiative acceleration could be even larger. Overall, we expect that the outer optically-thin layers would adopt a steeper density distribution and a faster-than-linear velocity distribution with radius. We plan to investigate these issues more quantitatively in the future, but at present, it seems that the earliest observations of SNe 2005cs and 2006bp support the very steep density distribution obtained in radiation-hydrodynamics simulations of core-collapse SN ejecta soon after shock breakout.

Before closing this section, we note that SN 1999gi is another Type II-P SN discovered very early, perhaps only $\sim 4_{-3.1}^{+3.0}$ days after explosion (Leonard et al. 2002b). Interestingly, Leonard et al. report the presence of a full P-Cygni profile shifted from the rest wavelength of $\mathrm{H} \beta$ by $-30000 \mathrm{~km} \mathrm{~s}^{-1}$ in the first spectrum of SN 1999gi, and speculate on the potential association with ejecta inhomogeneities. In the context of a density inhomogeneity far above the photosphere, a blueshifted absorption is a possibility, but a P-Cygni profile entirely blueshifted, and by that amount, is not (see, however, Dessart \& Hillier 2005a). We reproduce their observation in Fig. 15. and overplot the first spectrum of SN 2006bp (red line). Given the close correspondence between the two spectra and the likely early detection of SN 1999gi, we propose that, in fact, the broad line feature just blueward of $\mathrm{H} \beta$ in the spectrum of SN 1999gi is due to HeII 4686A, as for SN 2006bp.

\subsection{Energetics}

Having inferred the reddening and the distance to $\mathrm{SNe}$ $2005 \mathrm{cs}$ and $2006 \mathrm{bp}$, we can deduce the absolute magnitude for each event at all epochs studied. Together with results presented for SN 1999em in DH06, we show in the top-left panel of Fig. [16 the evolution with time since explosion of the absolute magnitude in $B$ (black), $V$ (blue), and $I$ (red), for SN 2005cs (solid line), SN 2006bp (dashed line; $i^{\prime}$ is shown instead of $I$ ), and SN $1999 \mathrm{em}$ (dotted line). Note that the time of explosion is computed together with the distance, rather than estimated based on prior non-detection of the SN on pre-explosion images of the corresponding host-galaxy ${ }^{13}$. While the

\footnotetext{
13 Our inferences are, however, compatible with these alternate estimates, in particular for SN 2006bp for which the explosion time is very tightly constrained by a non-detection on April 9.15 and
} 

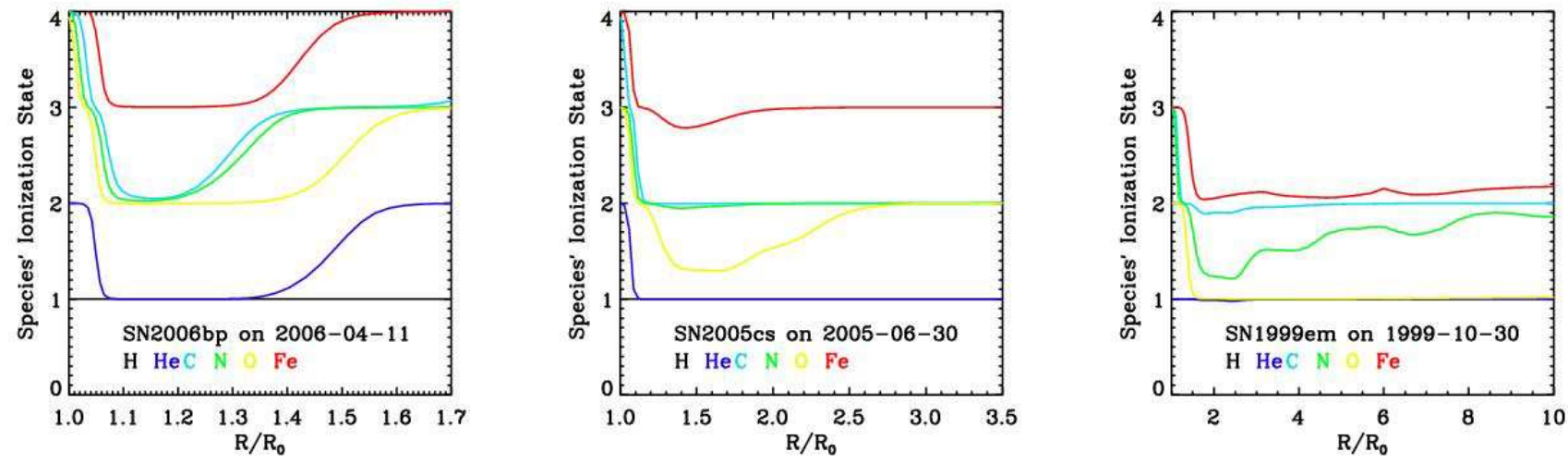

FIG. 14.- Radial variation of the ionization state of $\mathrm{H}$ (black), He (blue), C (turquoise), N (green), O (yellow), and Fe (red) in the ejecta of SN 2006bp (left; $\left.R_{0}=2.2 \times 10^{14} \mathrm{~cm}\right)$, SN $2005 \mathrm{cs}$ (middle; $R_{0}=1.6 \times 10^{14} \mathrm{~cm}$ ), and SN $1999 \mathrm{em}\left(\right.$ right; $\left.R_{0}=4.176 \times 10^{14} \mathrm{~cm}\right)$ based on the modeling of the first spectroscopic observation (which corresponds to 3 (\$5 and Fig. 8), 5 (\$4 and Fig. 31), and 6 (DH06 and their Fig. 1) days after explosion) and limited to the regions exterior to the layer where the inward integrated Rosseland optical depth is $\sim 50$ on that day. Note the systematic decrease of the ejecta ionization state as we go from SN 2006bp, to SN 2005cs, and to SN 1999em, following the trend of elapsed time since explosion. This clarifies the identified emission in He II $4686 \AA$ line in the first spectrum of SN 2006bp. Note also the rise in ionization at large distances (together with a rise of the temperature, not shown here), a non-LTE effect in these scattering-dominated environments. [See the electronic edition of the Journal for a color version of this figure, and see 7.1 for discussion.]

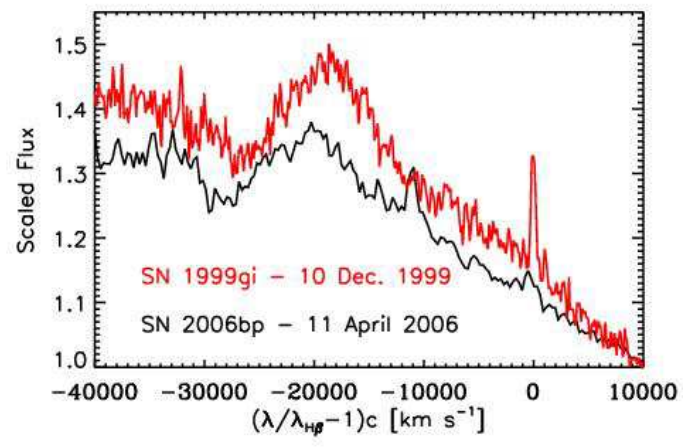

Fig. 15.- Comparison between the first spectroscopic observation of SN 2006bp (11th of April 2006) and of SN 1999gi (10 December 1999; Leonard et al. 2002b) showing the observed flux versus the Doppler velocity, with respect to the rest wavelength of $\mathrm{H} \beta$ (Note that the inferred reddening of SN 1999gi is $E(B-V)=0.21$, so about half what we infer for SN 2006bp; Leonard et al. 2002b). The P-Cygni profile seen in the SN 2006bp spectrum is identified as HeII 4686A (Fig. 8), and, given the similarity in profile shape and position, suggests a similar identification for SN 1999gi. [See the electronic edition of the Journal for a color version of this figure, and see 7.1 for discussion.]

spectroscopic and photometric evolution of three events are comparable in all spectral regions at a given time after explosion, they display a sizable range of intrinsic brightness. SN 2005cs is about two magnitudes fainter than SN 2006bp, about a factor of six in (bolometric) luminosity, with SN $1999 \mathrm{em}$ somewhat less luminous than SN 2006bp. SN 2006bp was detected earliest of all three and is in fact the first Type II-P to be caught so soon after explosion, with a $\sim 2$-day delay at most (note that 1987A and 1993J are not Type II-P SNe).

In the top-right panel of Fig. 16, we show the corresponding evolution of the model photospheric velocity, for SNe 2005cs, $1999 \mathrm{em}$, and 2006bp. The above trend is maintained, i.e., larger intrinsic brightness is associated with larger ejecta velocity. Note that if the bolometric luminosity scaled with the square of the ejecta velocity,

detection on April 10.15. a brightening by $1.5 \mathrm{mag}$ would result from a factor of 2 enhancement in velocity, which is compatible with our inferences for SNe 2005cs and 2006bp (top row of Fig. 16). Since we adopt similar ejecta structures (after one week past explosion), this suggests that the bolometric luminosity correlates with the ejecta kinetic energy in the hydrogen shell, as pointed out in the past by, for example, Falk \& Arnett (1977), Litvinova \& Nadezhin (1983), Hamuy (2003), Zampieri et al. (2003), Pastorello et al. (2004). The late time brightness is not controlled by the energy deposited at shock passage, but rather stems from the amount and location of the unstable nickel at the start of homologous expansion, so the above trend may not carry over until much later times, although it might, but for different reasons (higher energy explosions may systematically produce more ${ }^{56} \mathrm{Ni}$ ).

Similarly, and for completeness, we include in the bottom panels of Fig. 16 the temporal evolution of the photospheric radius (left) and temperature (right). Note the smaller extent of the slowly-expanding ejecta of SN2005cs, the converging photospheric temperatures, and the near-constant photospheric radii at the recombination epoch for all three SNe, tightly linked to the Plateau appearance of the visual light-curves at such times.

\subsection{A common origin for the $U V$ and the optical flux?}

Our modeling approach has been first to fit the optical range, using both the overall shape of the SED and spectral lines formed in the SN ejecta. As a consistency check, as well as an additional constraint on the resulting model parameters and the choice of reddening, we compared the synthetic spectrum to the observed Swift UVOT values. For both SN 2005cs and SN 2006bp, we obtain very satisfactory fits and we thus conclude that the observed UV and optical light emerges from the photospheric layers of the SN ejecta, over the first 1-2 months after explosion (starting 3-5 days afterwards). This result is unambiguous, and, thus, no sizable contribution to the UV and optical fluxes stems from an interaction with the CSM. 

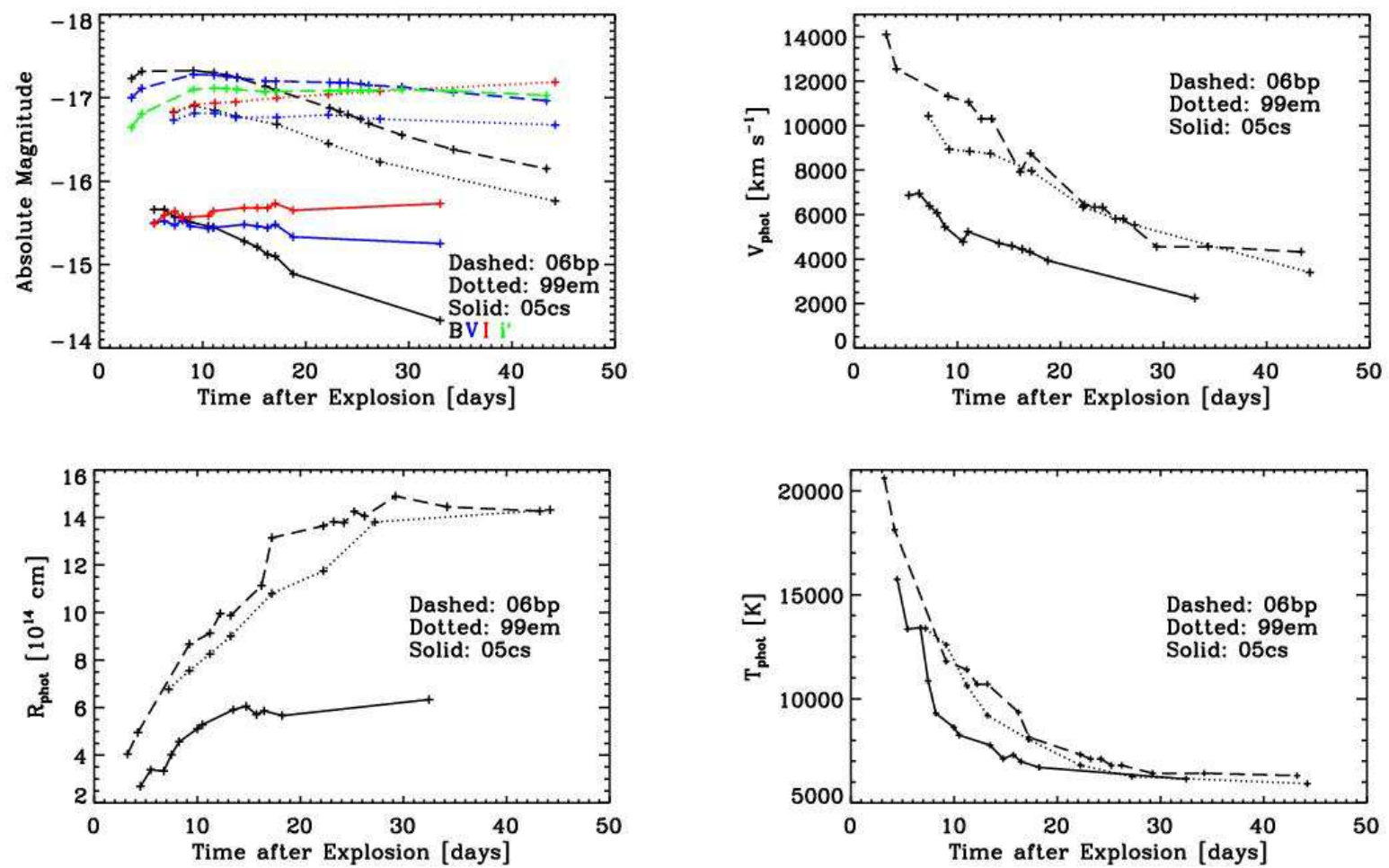

FIG. 16. - Top Left: Evolution of the absolute magnitude in the $B$ (black), $V$ (blue), and $I$ (red) bands for SN 2005cs (solid), SN 2006bp (dashed; $i^{\prime}$ is shown instead of $I$ ), and SN 1999em (dotted), with respect to the inferred time of explosion (JD 2453547.6 for SN 2005cs; JD 2453833.4 for SN 2006bp; JD 2451474.8 for SN 1999em), and the inferred distance of 8.9 Mpc and reddening of 0.04 for SN $2005 \mathrm{cs}$, $11.5 \mathrm{Mpc}$ and 0.1 for SN $1999 \mathrm{em}$, and $17.5 \mathrm{Mpc}$ and 0.4 for SN 2006bp. Note how the SN 2005cs is intrinsically underluminous compared to SN $1999 \mathrm{em}$, by about $1.5 \mathrm{mag}$ equivalent to a factor of four in luminosity (a valid comparison since their SED is comparable at a given time after explosion). SN 2006bp is somewhat more luminous than SN 1999em, by about $0.5 \mathrm{mag}$ in each bandpass. Crosses refer to the times of the observations that we use here, and given with respect to the inferred time of explosion obtained in this work and in DH06 for SN 1999em. Top Right: Same as left, but this time for the photospheric velocity. Notice how the relationship in absolute magnitude carries over for the photospheric velocity (uncertain at the $10 \%$ level), at the same time after explosion (note that the three ejecta have a similar density structure beyond one week after explosion). The larger the kinetic energy, the larger the bolometric luminosity of the ejecta. Bottom: Same as the top right panel, but for the photospheric radius (left) and temperature (right). [See the electronic edition of the Journal for a color version of this figure, and see 77.2 for discussion.]

A further evidence for the photospheric origin of the UV flux is the identification of the Mg II $2800 \AA$ line as a broad P-Cygni profile in Swift UVOT grism spectra (Brown et al. 2007), with a width compatible with the photospheric velocity at the corresponding epoch. So, while circumstellar interaction may be important in some Type II SNe (the IIn events), it seems to contribute negligibly to the UV and optical light we received from SN $2005 \mathrm{cs}$ and SN 2006bp, and more generally in agreement with the weak radio and X-ray emission from Type II-P $\mathrm{SNe}$ (see, e.g., Pooley et al. 2002; Chevalier et al. 2006).

\subsection{Comparison with previous work}

The recent spectroscopic analysis by Baron et al. (2007) of SN 2005cs at two epochs in the photospheric phase agrees in many respects with the findings of the present study for that object and, thus, gives some credence to the Type II SN spectroscopic analyzes performed with PHOENIX (Hauschildt \& Baron 1999) and CMFGEN (Hillier \& Miller 1998; Dessart \& Hillier 2005a). This is particularly satisfying since PHOENIX and CMFGEN are two very distinct codes, and use different assumptions in the modeling. A potential important difference in assumptions is our neglect of energy contribution from radioactive decay throughout this work. By contrast with Baron et al. (2007), we find no strong evidence for nickel mixing and non-thermal energy deposition at the photosphere over the first month after explosion (in this work, we obtain good fits to observations prior to the hydrogen-recombination epoch without invoking this additional source of energy). The ejecta ionization structure computed by the two codes may thus differ.

In this work, we infer a reddening $E(B-V) \sim 0.04$, in agreement with Baron et al. (2007) who obtain a value of 0.035 . We also concur with the findings of Baron et al. (2007) on the necessary CNO chemistry to reproduce the observations of $\mathrm{O}$ II and $\mathrm{N}$ II lines in the early spectra of SN 2005cs (as well as in SN 2006bp), with a depletion of carbon and oxygen compared to solar that is modest rather than severe. As discussed in 77.1 this constraint can be placed for SN 2005cs, by contrast with, e.g., SN $1999 \mathrm{em}$, mostly because it was observed at an earlier time when the ionization conditions at the photosphere are ripe for OII line emission. The identification of OII and NiI lines can explain most, if not all, the mysterious kinks seen in the blue wing of $\mathrm{H} \beta$ or HeI $5875 \AA$ (see, e.g., Leonard et al. 2002a,b who reported such kinks in the spectra of $\mathrm{SNe}$ $1999 \mathrm{em}$ and 1999gi). Here, the abundances adopted at early times for both $\mathrm{SNe} 2005 \mathrm{cs}$ and 2006bp are, given as mass fractions, $X_{\mathrm{H}}=0.55, X_{\mathrm{He}}=0.44, X_{\mathrm{C}}=0.0005$, 
$X_{\mathrm{N}}=0.002, X_{\mathrm{O}}=0.003$, and $X_{\mathrm{Fe}}=0.0013$. At later times, when in particular O I $7770 \AA$ gains in strength, we enhance both the carbon and the oxygen abundances, with $\mathrm{C} / \mathrm{He}=0.001, \mathrm{O} / \mathrm{He}=0.01$, and reduce the nitrogen abundance, $\mathrm{N} / \mathrm{He}=0.001$. Again, observations support the same ejecta chemistry at the photosphere at corresponding epochs after explosion for both SNe. We are however reserved concerning the sodium abundance, which we predicted, based on steady-state CMFGEN models, to be enhanced by a factor of four over its primordial value in SN 1999em (DH06). Comparable enhancements would be needed here for $2005 \mathrm{cs}$ and $2006 \mathrm{bp}$, and, indeed, Baron et al. (2007) predict a sodium enhancement of a factor of 10. Time-dependent CMFGEN models performed with a primordial sodium abundance, however, suggest that NaID may remain as strong and broad as observed (Dessart \& Hillier 2007a,b); further work is needed to quantify the effect of time-dependence on abundance determinations.

For the observations of the 14th of July 2005, Baron et al. (2007) find at the photosphere a temperature of $6000 \mathrm{~K}$, a velocity of $4000 \mathrm{~km} \mathrm{~s}^{-1}$ and a density exponent of 8 . In the same order and for that date, we find $6700 \mathrm{~K}$, $3930 \mathrm{~km} \mathrm{~s}^{-1}$, and 10, in agreement with their values. For the observations of the 31st of July 2005, Baron et al. find at the photosphere a temperature of $5500 \mathrm{~K}$, a velocity of $6000 \mathrm{~km} \mathrm{~s}^{-1}$ and a density exponent of 12 (see their \$6). In this work, for the observations of the 28th of July 2005, just three days before (note that observations do not change visibly over just a few days at such late times), we find in the same order $6140 \mathrm{~K}, 2240 \mathrm{~km} \mathrm{~s}^{-1}$ and a density exponent of 10 . While we fit the CfA observations reasonably well with this parameter set on that date (Fig,7), Baron et al. require a photospheric velocity nearly three times as large to do so, and also larger than the value on Day 17 (in fact nearly as large as on the first spectrum taken, for which we use $6880 \mathrm{~km} \mathrm{~s}^{-1}$ ). Upon inspection of their Figs. 12-13, it appears that $\mathrm{H} \alpha$ peaks at $\sim 6480 \AA$, while the observations they use, as originally published in P06, and our (restframe) CfA observations show a peak at $6550 \AA$. This difference of $70 \AA$ corresponds to a velocity shift of $3200 \mathrm{~km} \mathrm{~s}^{-1}$ at this wavelength and suggests that their extravagant proposition of an outward moving photosphere between Day 17 and Day 34 (see their §6) may stem solely from a problem with the wavelength of their flux datapoints.

Baron et al. (2007) obtained a SEAM distance to SN 2005 cs of $7.9_{-0.6}^{+0.7} \mathrm{Mpc}$ using all $U B V R I$ magnitudes, but at only two epochs, which is in agreement with our estimate of $8.9 \pm 0.5 \mathrm{Mpc}$ using $B V I$ and 12 or 13 epochs.

Although the observed spectra of SNe 2005cs and 2006 bp presented in this work are different in many ways, these reflect mostly the difference in the ejecta expansion rate (small for $2005 \mathrm{cs}$ ), the reddening in the line of sight (large for 2006bp), and the elapsed time between explosion and discovery (very short for 2006bp). As shown in Fig. 16, both SNe evolve in a similar fashion, 2005cs being merely underluminous and boasting a lower ejecta kinetic energy.

\section{CONCLUSION}

We have presented a multi-epoch multi-wavelength quantitative spectroscopic analysis of the two Type II-P SN events $2005 \mathrm{cs}$ and $2006 \mathrm{bp}$, over the first two months after explosion, and have reached similar conclusions on the ejecta properties to those for SN 1999em (DH06). The progenitor stars have a composition compatible with a BSG/RSG progenitor. The spectral evolution shows a reddening of the SED with time, with a flux peaking in the UV at very early times, but progressively shifting to longer wavelengths. The photosphere recedes to deeper layers with time, reaching mass shells moving at about 2000-3000 $\mathrm{km} \mathrm{s}^{-1}$ about 6 weeks after explosion and having enhanced carbon and oxygen, and reduced nitrogen, abundances, compared to the surface layers. We find a density distribution that is very steep at early times, and we surmise that this could be an imprint of the shock breakout epoch. From about a week until six weeks after explosion, we find a more standard and constant density exponent of ten for the corresponding power law distribution, compared to a value of 50 (for SN 2006bp) and 20 (for SN 2005cs) used to model the first observation. Swift UVOT photometry and ground-based optical spectroscopy and photometry support a common origin for the UV and the optical light, so that we anticipate that if CSM interaction does occur, it does not contribute significantly to the observed UV and optical fluxes.

Using our analysis, we infer the distance and the explosion date of each SN. We find, in that order, JD $2453547.6 \pm 0.5$ days (2.8 days prior to discovery) and $8.9 \pm 0.5 \mathrm{Mpc}$ for SN 2005cs, and JD 2453833.4 \pm 0.4 days (1.7 days prior to discovery) and $17.5 \pm 0.8 \mathrm{Mpc}$ for SN $2006 \mathrm{bp}$. The large number of observations, the quality of our fits, the agreement between the two methods followed suggests a very high level of internal consistency of the distances determined here with Type II SNe. The actual level of accuracy on such determined distances, which we estimate to be on the order of $10 \%$ (related to the spectral fitting accuracy), would be best assessed by confrontation with alternate methods (Cepheids for example, although all alternate methods have their own inaccuracies, claimed and otherwise), the determination of distances to a galaxy host with well-recorded multiple Type II SN events (the typical Type II SN rate of 2 per century per galaxy makes this proposition somewhat unrealistic), a Galactic SN that could also be resolved with an interferometer or a large-aperture telescope. Our perspective on the uncertainty of our distance measurements is that, were we to increase the number of Type II SNe suitable for analysis in M 51 to 10 or 100, the scatter in the distance determined with our technique for that sample would not be larger than $10 \%$. But this would still be no proof that the resulting average distance is indeed the right one since enlarging the sample would only allow for an internal consistency check on our method. In the short term, a Cepheid distance to the galaxy host of $2005 \mathrm{cs}$ and $2006 \mathrm{bp}$ is highly desirable, and would provide an independent check on the distance computed with Type II-P SNe, as was done with success in the past with SN 1999em (Leonard et al. 2003; Baron et al. 2004; DH06), giving further support to the potential use of such objects for distance determinations in the Universe.

This work fits within an ambitious project to build a general understanding of SN ejecta, to infer properties of the progenitor star (with ramifications for massive star evolution) and the explosion mechanisms at the origin of the event. A major ongoing theoretical development is 
the treatment of time-dependent effects both in the radiation field and in the level populations to allow for a direct modeling of the evolution over months after shock breakout, through the photospheric and into the nebular phases, and based on a range of hydrodynamical models of the explosion. In parallel, high-quality multi-epoch multi-wavelength observations for a substantial number of nearby SNe, irrespective of the type, galaxy host properties, covering from as soon as possible after explosion until the nebular phase, is eagerly sought.

We thank Stan Woosley for providing the ejecta structure of a $15 \mathrm{M}_{\odot}$ model. We also thank Andrea Pa- storello, Robert Quimby, and Doug Leonard for providing their optical spectra of SN 2005cs, SN 2006bp, and SN 1999gi, respectively. L.D. acknowledges support for this work from the Scientific Discovery through Advanced Computing (SciDAC) program of the DOE, under grant numbers DE-FC02-01ER41184 and DE-FC0206ER41452, and from the NSF under grant number AST0504947. This research was supported in part by the National Science Foundation under Grant No. PHY0551164 to the Kavli Institute for Theoretical Physics at UC Santa Barbara and AST-0606772 to Harvard University. This work is sponsored at PSU by NASA contract NAS5-00136.

\section{REFERENCES}

Aldering, G., et al. 2005, The Astronomer's Telegram, 667, 1

Baron, E., Hauschildt, P. H., Branch, D., Kirshner, R. P., \& Filippenko, A. V. 1996, MNRAS, 279, 799

Baron, E., et al. 2000, ApJ, 545, 444

Baron, E., Nugent, P. E., Branch, D., Hauschildt, P. H., Turatto, M., \& Cappellaro, E. 2003, ApJ, 586, 1199

Baron, E., Nugent, P. E., Branch, D., \& Hauschildt, P. H. 2004, ApJ, 616, L91

Baron, E., Branch, D., \& Hauschildt, P. H. 2007, ApJ, 662, 1148

Blondin, S., et al. 2006, AJ, 131, 1648

Blinnikov, S., Lundqvist, P., Bartunov, O., Nomoto, K., \& Iwamoto, K. 2000, ApJ, 532, 1132

Brown, P. J., et al. 2007, ApJ, 659, 1488

Cardelli, J.A., Clayton, G.C., \& Mathis, J.S. 1988, ApJ, 329, 33

Castor, J. I., Abbott, D. C., \& Klein, R. I. 1975, ApJ, 195, 157

Chevalier, R. A. 1982, ApJ, 259, 302

Chevalier, R. A., Fransson, C., \& Nymark, T. K. 2006, ApJ, 641, 1029

Chugai, N. N., et al. 2004, MNRAS, 352, 1213

Chugai, N. N., Chevalier, R. A., \& Utrobin, V. P. 2007, ApJ, 662, 1136

Ciardullo, R., Feldmeier, J. J., Jacoby, G. H., Kuzio de Naray, R., Laychak, M. B., \& Durrell, P. R. 2002, ApJ, 577, 31

Crowther, P. A., Dessart, L., Hillier, D. J., Abbott, J. B., \& Fullerton, A. W. 2002, A\&A, 392, 653

Crowther, P. A., Lennon, D. J., \& Walborn, N. R. 2006, A\&A, 446, 279

Dessart, L., Crowther, P. A., Hillier, D. J., Willis, A. J., Morris, P. W., \& van der Hucht, K. A. 2000, MNRAS, 315, 407

Dessart, L., \& Hillier, D. J. 2005a, A\&A, 437, 667

Dessart, L., \& Hillier, D. J. 2005b, A\&A, 439, 671

Dessart, L., \& Hillier, D. J. 2006a, A\&A, 447, 691 (DH06)

Dessart, L., \& Hillier, D. J. 2007a, American Institute of Physics Conference Series, 924, 441

Dessart, L., \& Hillier, D. J. 2007b, accepted to MNRAS, ArXiv e-prints, 710 , arXiv:0710.0784

Eastman, R. G., \& Kirshner, R. P. 1989, ApJ, 347, 771

Eastman, R. G., Schmidt, B. P., \& Kirshner, R. 1996, ApJ, 466, 911

Eisenstein, D. J., et al. 2005, ApJ, 633, 560

Elias-Rosa, N., et al. 2006, MNRAS, 369, 1880

Elmhamdi, A., et al. 2003, MNRAS, 338, 939

Ensman, L., \& Burrows, A. 1992, ApJ, 393, 742

Falk, S. W., \& Arnett, W. D. 1977, ApJS, 33, 515

Fabricant, D., Cheimets, P., Caldwell, N., \& Geary, J. 1998, PASP 110,79

Feldmeier, J. J., Ciardullo, R., \& Jacoby, G. H. 1997, ApJ, 479, 231

Filippenko, A. V. 1982, PASP, 94, 715

Filippenko, A. V., et al. 1995, ApJ, 450, L11

Fox, D. W., et al. 2000, MNRAS, 319, 1154

Fransson, C., \& Kozma, C. 1993, ApJ, 408, L25

Garnavich, P. M., et al. 1998, ApJ, 509, 74

Gehrels, N., et al. 2004, ApJ, 611, 1005

Georgiev, Ts. B., Getov, R. G., Zamanova, V. I., \& Ivanov, G. R. 1990, Pis. Astron. Zh., 16, 979

Grevesse, N., \& Sauval, A. J. 1998, Space Science Reviews, 85, 161

Hachisu, I., Matsuda, T., Nomoto, K., \& Shigeyama, T. 1990, ApJ, $358, \mathrm{~L} 57$
Hachisu, I., Matsuda, T., Nomoto, K., \& Shigeyama, T. 1991, ApJ, 368, L27

Hamuy, M., et al. 2001, ApJ, 558, 615

Hamuy, M., \& Pinto, P. A. 2002, ApJ, 566, L63

Hamuy, M. 2003, ApJ, 582, 905

Hauschildt \& Baron 1999, J. Comp. Applied Math., 109, 41

Hillier, D. J., \& Miller, D. L. 1999, ApJ, 519, 354

Hillier, D. J., Lanz, T., Heap, S. R., Hubeny, I., Smith, L. J., Evans, C. J., Lennon, D. J., \& Bouret, J. C. 2003, ApJ, 588, 1039

Hicken, M. 2007, in preparation

Hillier, D. J., \& Miller, D. L. 1998, ApJ, 496, 407

Hoëflich, P. 1988, Proceedings of the Astronomical Society of Australia, 7, 434

Ho, L. C., Filippenko, A. V., \& Sargent, W. L. W. 1997, ApJS, 112,315

Horne, K. 1986, PASP, 98, 609

Immler, S., et al. 2007, ApJ, 664, 435

Imshennik, V. S., \& Nadezhin, D. K. 1988, Soviet Astronomy Letters, 14,449

Iwamoto, K., Nomoto, K., Hoflich, P., Yamaoka, H., Kumagai, S., \& Shigeyama, T. 1994, ApJ, 437, 115

Kifonidis, K., Plewa, T., Janka, H.-T., Mueller, E. 2000, ApJ, 531, L123

Kifonidis, K., Plewa, T., Janka, H.-T., Mueller, E. 2003, A\&A, 408, 621

Kirshner, R. P., Oke, J. B., Penston, M. V., \& Searle, L. 1973, ApJ, 185,303

Kirshner, R. P., \& Kwan, J. 1974, ApJ, 193, 27

Kirshner, R. P., \& Kwan, J. 1975, ApJ, 197, 415

Kloehr, W., Muendlein, R., Li, W., Yamaoka, H., \& Itagaki, K. 2005, IAU Circ., 8553, 1

Kozma, C., \& Fransson, C. 1998a, ApJ, 496, 946

Kozma, C., \& Fransson, C. 1998b, ApJ, 497, 431

Kumagai, S., Shigeyama, T., Nomoto, K., Itoh, M., Nishimura, J., \& Tsuruta, S. 1989, ApJ, 345, 412

Leonard, D. C., et al. 2002a, PASP, 114, 35

Leonard, D. C., et al. 2002b, AJ, 124, 2490

Leonard, D. C., Kanbur, S. M., Ngeow, C. C., \& Tanvir, N. R. 2003, ApJ, 594, 247

Leonard, D. C., \& Filippenko, A. V. 2001, PASP, 113, 920

Li, W., Van Dyk, S. D., Filippenko, A. V., Cuillandre, J.-C., Jha, S., Bloom, J. S., Riess, A. G., \& Livio, M. 2006, ApJ, 641, 1060

Litvinova, I. I., \& Nadezhin, D. K. 1983, Ap\&SS, 89, 89

Macri, L. M., Stanek, K. Z., Bersier, D., Greenhill, L. J., \& Reid, M. J. 2006, ApJ, 652, 1133

Matheson, T., Filippenko, A. V., Ho, L. C., Barth, A. J., \& Leonard, D. C. 2000, AJ, 120, 1499

Matzner, C. D., \& McKee, C. F. 1999, ApJ, 510, 379

Maund, J. R., Smartt, S. J., \& Danziger, I. J. 2005, MNRAS, 364, L33

Matheson, T., et al. 2000, AJ, 120, 1487

Mitchell, R. C., Baron, E., Branch, D., Lundqvist, P., Blinnikov, S., Hauschildt, P. H., \& Pun, C. S. J. 2001, ApJ, 556, 979

Mitchell, R. C., Baron, E., Branch, D., Hauschildt, P. H., Nugent, P. E., Lundqvist, P., Blinnikov, S., \& Pun, C. S. J. 2002, ApJ, 574,293

Modjaz, M., Kirshner, R., Challis, P., \& Hutchins, R. 2005, IAU Circ., 8555, 1

Nakano, S., \& Itagaki, K. 2006, IAU Circ., 8700, 4 
Pastorello, A., et al. 2002, MNRAS, 333, 27

Pastorello, A., et al. 2004, MNRAS, 347, 74

Pastorello, A., et al. 2006, MNRAS, 370, 1752 (P06)

Pinto, P. A., \& Woosley, S. E. 1988, Nature, 333, 534

Poole, T. S., et al. 2007, ArXiv e-prints, 708, arXiv:0708.2259

Pooley, D., et al. 2002, ApJ, 572, 932

Quimby, R., Brown, P., Caldwell, J., \& Rostopchin, S. 2006, Central Bureau Electronic Telegrams, 471, 1 (2006). Edited by Green, D. W. E., 471, 1

Quimby, R. M., Wheeler, J. C., Höflich, P., Akerlof, C. W., Brown, P. J., \& Rykoff, E. S. 2007, ApJ, 666, 1093

Richmond, M. W., Filippenko, A. V., \& Galisky, J. 1998, PASP, 110,553

Riess, A. G., et al. 2005, ApJ, 627, 579

Roming, P. W. A., et al. 2005, Space Sci. Rev., 120, 95

Salamanca, I., Terlevich, R. J., \& Tenorio-Tagle, G. 2002, MNRAS, 330,844

Schechter, P. L., Mateo, M., \& Saha, A. 1993, PASP, 105, 1342

Schmidt, B. P., Kirshner, R. P., Eastman, R. G., Phillips, M. M., Suntzeff, N. B., Hamuy, M., Maza, J., \& Aviles, R. 1994, ApJ, 432,42

Schmutz, W., Abbott, D. C., Russell, R. S., Hamann, W.-R., \& Wessolowski, U. 1990, ApJ, 355, 255

Shigeyama, T., \& Nomoto, K. 1990, ApJ, 360, 242
Spergel, D. N., et al. 2007, ApJS, 170, 377

Takáts, K., \& Vinkó, J. 2006, MNRAS, 372, 1735

Tonry, J. L., Dressler, A., Blakeslee, J. P., Ajhar, E. A., Fletcher, A. B., Luppino, G. A., Metzger, M. R., \& Moore, C. B. 2001, ApJ, 546, 681

Tully, R. B. 1988, Cambridge and New York, Cambridge University Press, 1988, 221 p.,

Tsvetkov, D. Y., Volnova, A. A., Shulga, A. P., Korotkiy, S. A., Elmhamdi, A., Danziger, I. J., \& Ereshko, M. V. 2006, A\&A, 460,769

Utrobin, V. P., \& Chugai, N. N. 2005, A\&A, 441, 271

Utrobin, V. P. 2007, A\&A, 461, 233

Verheijen, M. A. W., \& Sancisi, R. 2001, A\&A, 370, 765

Wade, R. A., \& Horne, K. 1988, ApJ, 324, 411

Wheeler, J. C., Harkness, R. P., Clocchiatti, A., Benetti, S., Brotherton, M. S., Depoy, D. L., \& Elias, J. 1994, ApJ, 436, L135

Woosley, S., \& Janka, T. 2005, Nature Physics, 1, 147

Woosley, S. E., \& Heger, A. 2007, Phys. Rep., 442, 269

Zampieri, L., Pastorello, A., Turatto, M., Cappellaro, E., Benetti, S., Altavilla, G., Mazzali, P., \& Hamuy, M. 2003, MNRAS, 338, 711 


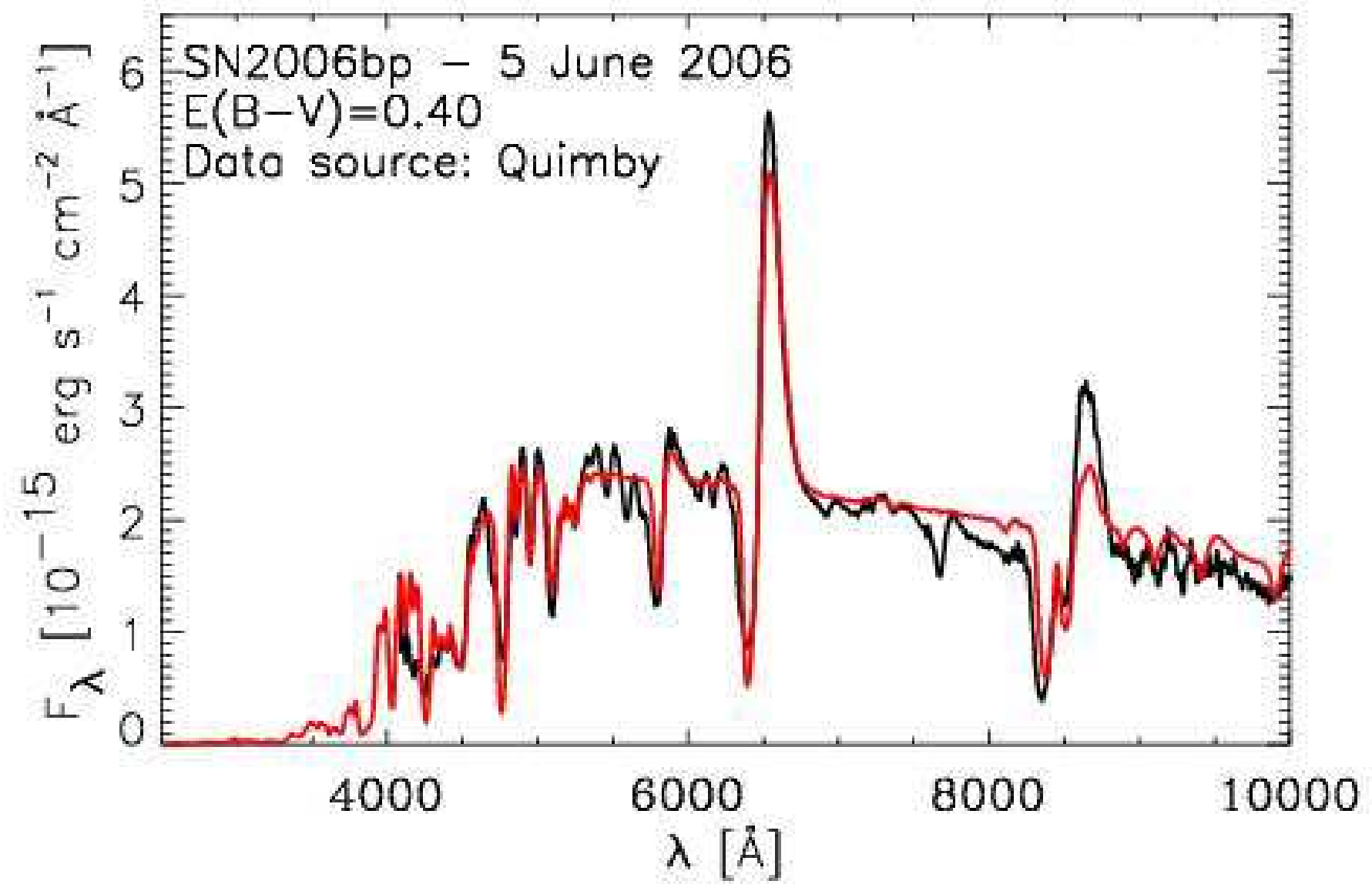

